\title{
A Contribution on Stochastic Optimal Control to Quantitative Finance
}

Effendi Syahril

Department of Mathematics

Bogor Agricultural University 


\begin{abstract}
This work considers a consumption and investment decision problem for an individual who has available a riskless asset paying fixed interest rate and a risky asset driven by Brownian motion price fluctuations. The individual is supposed to observe his or her current wealth only, when making transactions, that transactions incur costs, and that decisions to transact can be made at any time based on all current information. The transactions costs under consideration could be a fixed, linear or a nonlinear function of the amount transacted. In addition, the investor is charged a fixed fraction of total wealth as management fee. The investor's objective is to maximize the expected utility of consumption over a given horizon.

On the basis of this model, the existence of an optimal solution is given. Optimal consumption and investment strategies are obtained in closed form for each type of transaction costs function. In addition, the optimal interval of time between transactions is also derived. Results show that, for each transaction cost, transaction interval satisfies a nonlinear equation, which depends on total wealth at the beginning of that intervals. If, at each transaction, there is no costs involved other than that of management fee which is a fixed fraction of current portfolio value, then the optimal interval of time between transactions is fixed, independent of time and current wealth.
\end{abstract}


This work is dedicated to the memory of my parents Syahril Sutan Ma'ruf and Syamsiar 


\section{Acknowledgment}

I am indebted to several institutions and individuals for their supports and encouragement during the period of study for this work.

I would like to thank my colleagues in the Department of Mathematics, Bogor Agricultural University, for the support and encouragement I have received during the period of study for this work.

Scholarship from Australian Government through Indonesian Government is very much appreciated. My thank goes to administration staffs of AusAID (Jakarta office as well as Adelaide office) for the arrangement during the period of study for this work.

I would like to thank the administration of The Department of Applied Mathematics, The University of Adelaide, for giving me an opportunity to work on this project. My deepest thank goes to Dr. John van der Hoek at The Department of Applied Mathematics, The University of Adelaide, for the many useful discussions over the years and his enthusiasm for the research contained within this work.

Finally, I would like to thank all members of my family, for all hopes, supports and encouragement during the period of this work. 


\section{DECLARATION}

I hereby declare that this work contains no material previously published or written by any person, except where due reference is made in the text of the work.

Effendi Syahril 


\section{Contents}

1 Introduction $\quad 1$

2 Formulation of the Model 5

2.1 Introduction . . . . . . . . . . . . . . 5

2.2 Assumptions And Notations . . . . . . . . . . . . . . . 6

2.2 .1 Uncertainty . . . . . . . . . . . . . 6

2.2 .2 Security Markets . . . . . . . . . . . . . 7

2.2 .3 Transaction costs . . . . . . . . . . . . 8

2.2 .4 Information . . . . . . . . . . . . . 10

2.3 The Model . . . . . . . . . . . . . . . . . . . . . . . . 11

2.3.1 Preferences ................... 11

2.3.2 Feasible policies . . . . . . . . . . . . . 11

2.4 The Problem . . . . . . . . . . . . . . . . . . 13

3 Portfolio Selection with Bullet Transaction Costs 14

3.1 Introduction . . . . . . . . . . . . . . . . . . . 14

3.2 Statement of the Problem . . . . . . . . . . . . . 16

3.3 Consumption Strategy f . . . . . . . . . . . . . 16 
3.4 Investment Strategies . . . . . . . . . . . . . . . . 24

3.5 Optimal Transaction Intervals . . . . . . . . . . . . 35

3.6 Equal Intervals . . . . . . . . . . . . . . . . . 45

4 Portfolio Selection with Linear Transaction Costs 48

4.1 Introduction . . . . . . . . . . . . . . . . . . 48

4.2 Statement of the Problem . . . . . . . . . . . . . 50

4.3 Consumption Strategy . . . . . . . . . . . . . . . 50

4.4 Investment Strategies . . . . . . . . . . . . . . . . 55

4.5 Optimal Transaction Intervals . . . . . . . . . . . . . . 63

5 Portfolio Selection with Nonlinear Transaction Costs $\quad 73$

5.1 Introduction . . . . . . . . . . . . . . . . . 73

5.2 Statement of the Problem . . . . . . . . . . . . 75

5.3 Consumption Strategy _ . . . . . . . . . . . . . . . . 75

5.4 Investment Strategies . . . . . . . . . . . . . . . . . . . 81

5.5 Optimal Transaction Intervals . . . . . . . . . . . . . . 90

6 Conclusions 100 


\section{Chapter 1}

\section{Introduction}

Since the publication of Merton's seminal work [19], stochastic optimal control and stochastic calculus techniques have been widely applied to the area of finance. Robert. C. Merton iniatiated the study of financial markets using continuous-time stochastic models. Merton $[18,19]$ studied the behaviour of a single agent acting as a market price-taker who seeks to maximize expected utility of consumption. The utility function of the agent was assumed to be a power function, and the market was assumed to comprise a risk-free asset with constant rate of return and one or more stocks, each with constant mean rate of return and volatility. The only information available to the agent were current prices of the assets. There were no transaction costs. It was also assumed that the assets were divisible. In this idealized setting, Merton was able to derive a closed-form solution to the stochastic optimal control problem faced by the agent.

Since then, several authors have made contributions to the stochastic op- 
timal control and stochastic calculus analyses of the Merton's model. Among them are Constantinides [2, 3], Cox and Huang [4, 5], Davis and Norman [6], Duffie and Sun [7], Dumas and Luciano [8], Fleming and Zariphopoulou [10], Karatzas [13], Karatzas,Lehoczky, Sethi and Shreve [14], Lehoczky, Sethi and Shreve [15], Lelands [16], Magill and Constantinides [17], Sethi, Taksar and Presman [24], Shreve and Soner [25], Shreve, Soner and Xu [26], Taksar, Klass and Assaf [27], Zariphopoulou [28, 29]. This thesis contributes to the study of Merton's model with transaction costs.

The introduction of transaction costs to Merton's model was first accomplished by Magill and Constantinides [17]. Since then, several authors have published a number of works on Merton's model with transaction costs. To mention a few, they are Constantinides [2, 3], Davis and Norman [6], Duffie and Sun [7], Dumas and Luciano [8], Lelands [16], Shreve and Soner [25], Shreve, Soner and Xu [26], Taksar, Klass and Assaf [27], Zariphopoulou [29]. However, their works only concerning proportional transactions costs. As it was aknowledged in [17], the proportional transaction costs was less realistic than the transaction costs should be.

Davis and Norman in [6] and later Shreve and Soner in [25], studied optimal consumption and investment problem for an investor who has a bank account paying a fixed rate of interest and a stock whose price is lognormal diffusion, with proportional transaction costs. Their analysis relied heavily on the homothetic property of the value function for the proportional transaction costs. When transaction costs are nonlinear, the homothetic property no longer holds.

All of the works mentioned above were for continuous time models. Dis- 
crete time models were treated in Hakansson [11], Mossin [20], Pliska [21], Samuelson [23].

Duffie and Sun in [7] treated the proportional transaction costs with different formulation to others, which they call discrete continuous time formulation. Their formulation assumes that an investor observes current wealth when making transaction, and decisions to transact can be made at any time, but without no costs. They treated general linear transaction costs of the form $a W_{\tau_{n}}+b$, with $W_{\tau_{n}}$ denotes the amount of wealth transacted, and $a$ and $b$ are non-negatives. When $b=0$, we have proportional transaction costs, and the optimal transaction intervals are equal. However, no result given for $b \neq 0$. So, this thesis picks up where they left off : with more general transaction costs.

The main aim of this thesis is to establish a framework for the discretecontinuous-time formulation of Duffie and Sun in [7]. In pursuing that task, the discussion of this thesis starts with the fixed (bullet) transaction costs problem. It continues with a linear transaction costs problem, and ends with a nonlinear transaction costs problem. In other words, discussion develops from a simple costs function to a much more general function.

The organization of this thesis is as follows. The formulation of the model is given in Chapter 2. Chapter 3 discusses the optimal consumption and investment decisions when transaction costs function is fixed, regardless of the amount of wealth transacted. A complete optimal solution to the consumption and investment problem is established. Results show that the optimal interval of time between transactions depends on the total wealth at the beginning of each intervals. However, when a management fee is the only 
transaction cost apllying, then the optimal interval of time between transactions are equal. This result agrees with that of Duffie and Sun [7]. In Chapter 4, we extend the analysis of Chapter 3 to the consumption and investment selection problem with transaction costs consisting of fixed plus proportional transaction costs and a management fee. The existence of an optimal solution to that consumption and investment problem is given. In Chapter 5, we discuss the consumption and investment problem for an investor with nonlinear transaction costs plus management fees. We derived a complete optimal solution to this problem. The optimal value function as well as the optimal withdrawal process and investment strategy are given in closed forms, for given sequence of transactions intervals. Then, an equation satisfied by optimal transaction intervals is derived. This equation is of nonlinear type depending on the wealth at the beginning of that intervals. We show that, under appropriate restrictions, this equation has a solution, and that the solution indeed is optimal. We present conclusions in Chapter 6 . 


\section{Chapter 2}

\section{Formulation of the Model}

\section{$2.1 \quad$ Introduction}

Chapter 2 is concerned with the formulation of consumption and investment problem for an investor who seeks to maximize his/her expected utility of consumption. The investor is assumed to have a riskless and a risky securities in his/her portfolio. The investor observes his/her current wealth only when making transactions, transactions incur costs, and decisions to transact can be made at any time based on all current information. Transaction costs consists of management fee and costs of withdrawing wealth from the portfolio. Three forms of withdrawal costs will be considered : fixed, linear as well as nonlinear costs. The rest of Chapter 2 is organized as follows. We establish terminology as well as assumptions and notations in Section 2.2. The construction of the model is given in Section 2.3. Finally, we summarize our model in Section 2.4. 


\subsection{Assumptions And Notations}

Apart from some notational changes and types of transaction costs, the formulation in this thesis is as in Duffie and Sun [7].

\subsubsection{Uncertainty}

The following definitions on probability are standard. ${ }^{1}$ It is assumed that a complete probability space $(\Omega, \mathcal{F}, \mathcal{P})$ is given. In addition, it is assumed that a filtration $\left\{\mathcal{F}_{t}: t \geq 0\right\}$ is also given. By a filtration is meant a family of $\sigma$-algebras $\left\{\mathcal{F}_{t}: t \geq 0\right\}$ which is increasing : $\mathcal{F}_{s} \subset \mathcal{F}_{t}$ if $s \leq t$.

Definition 2.1 A filtered complete probability space $\left(\Omega, \mathcal{F},\left(\mathcal{F}_{t}\right)_{0 \leq t<\infty}, P\right)$ is said to satisfy the usual hypotheses if

1. $\mathcal{F}_{0}$ contains all the $P$-null sets of $\mathcal{F}$

2. the filtration $\left\{\mathcal{F}_{t}: t \geq 0\right\}$ is right continuous.

A stochastic process $X$ on $(\Omega, \mathcal{F}, \mathcal{P})$ is a collection of random variables $\left\{X_{t}: t \geq 0\right\}$. The process $X$ is said to be adapted if $X_{t}$ is $\mathcal{F}_{t}$ measurable for each $t$.

Definition 2.2 A process $B=\left\{B_{t}: t \geq 0\right\}$ adapted to $\left\{\mathcal{F}_{t}: t \geq 0\right\}$ taking values in $\mathbf{R}$ is called a one-dimensional standard Brownian motion if :

1. $B_{0}=0$, almost surely;

${ }^{1}$ Some notions in this part are derived from Protter [22] 
2. for $0 \leq s<t<\infty, B_{t}-B_{s}$ is independent of $\mathcal{F}_{s}$;

3. for $0<s<t, B_{t}-B_{s}$ is $N(0, t-s)$.

In this thesis, it is assumed that the one-dimensional standard Brownian motion $B=\left\{B_{t}: t \geq 0\right\}$ is given on a given filtered probability space $\left(\Omega, \mathcal{F},\left(\mathcal{F}_{t}\right)_{0 \leq t<\infty}, P\right)$

\subsubsection{Security Markets}

The following definition are as in Fabozzi [9] or Merton [18].

Definition 2.3 A riskless security is defined to be a security whose return in the future time is known with certainty. A risky security is one for which the return in the future is uncertain.

There are two securities available in the economy to an investor. One is a riskless security with fixed interest rate $r$, and the other is a risky security whose price is a geometric Brownian motion with expected rate of return $\alpha$ and rate of return variation $\sigma^{2}$. At time $t \geq 0$, the price processes $\left\{P_{0}(t)\right\}$ of the riskless security satisfy a deterministic differential equation :

$$
d P_{0}(t)=r P_{0}(t) d t
$$

while the price processes $\left\{P_{1}(t)\right\}$ of the risky security satisfy a stochastic differential equation :

$$
d P_{1}(t)=\alpha P_{1}(t) d t+\sigma P_{1}(t) d B_{t} .
$$

There is money available for the investor in the economy as a medium of exchange and numeraire. Only money is exchangable for consumption. Money 
can only be acquired by selling securities, it cannot be borrowed. Let $M_{t}$ denotes money holdings at time t. One unit of money can be exchanged at any time for one unit of consumption. The investor is assumed to receive no further income from noncapital sources, and starts with the initial stock of money $M_{0}=0$.

\subsubsection{Transaction costs}

Definition 2.4 A portfolio transaction consists of withdrawing wealth in the form of money from the investment portfolio in the securities and then adjusting the portfolio of securities.

Trading opportunities are available continuously in time, but not without costs. Transactions costs are incurred when information is processed and a portfolio transaction is made. There are two forms of transaction costs : portfolio management fees and withdrawal costs. The investor pays a fraction $\varepsilon>0$ of the total wealth in the securities at the beginning of each interval as a portfolio management fee. The portfolio management fee is meant to include the cost of adjusting the portfolio and the cost of processing information. For the purpose of analyses in this thesis, transactions costs is the costs which incurs during withdrawing wealth from the portfolio.

Definition 2.5 A transaction costs is meant the withdrawal costs, which is a function of amount of wealth withdrawn from the portfolio.

Three different types of transaction costs functions will be considered. The first type of transaction costs function which will be considered is a fixed 
(bullet) transaction costs function. Let $\Psi$ be a transaction costs function. If $W_{\tau_{n}}$ denotes the amount of wealth withdrawn at time $\tau_{n}$, then $\Psi$ is defined by

$$
\Psi\left(W_{\tau_{n}}\right)= \begin{cases}b>0, & \text { if } W_{\tau_{n}}>0 \\ 0, & \text { otherwise. }\end{cases}
$$

Then the total transaction costs function is of the form $b+\varepsilon\left(X_{\tau_{n}}-W_{\tau_{n}}\right)$, where $X_{\tau_{n}}$ is the total wealth at time $\tau_{n}$ before transaction.

The second type of transaction costs functions which will be considered is of linear function type. The transaction costs function $\Psi$ is defined by

$$
\Psi\left(W_{\tau_{n}}\right)= \begin{cases}a W_{\tau_{n}}+b, & \text { if } W_{\tau_{n}}>0 \\ 0, & \text { otherwise }\end{cases}
$$

where constants $a, b$ are nonnegative. The total transaction costs function is of the form $a W_{\tau_{n}}+b+\varepsilon\left(X_{\tau_{n}}-W_{\tau_{n}}\right)$.

The third type of transaction costs function considered involves a nonlinear, nondecreasing, and concave function of its argument. Having considered fixed and linear transaction costs functions, it is reasonable to consider a much more general type of transaction costs function. The transaction costs function $\Psi$ is defined by

$$
\Psi\left(W_{\tau_{n}}\right)= \begin{cases}\Phi\left(W_{\tau_{n}}\right), & \text { if } W_{\tau_{n}}>0 \\ 0, & \text { otherwise }\end{cases}
$$

where $\Phi$ is nonlinear, nondecreasing, concave and smooth function of $W_{\tau_{n}}$. 


\subsubsection{Information}

The Filtration $\left(\mathcal{F}_{t}\right)$ defined by $\mathcal{F}_{t}=\sigma\left\{B_{s}: s \leq t\right\}$, will be interpreted as information available up to time t. That is, measurability with respect to $\mathcal{F}_{t}$ is equivalent to measurability with respect to market information up to time $t$. Given the structure of transaction costs, consumption and investment decisions are made at intervals. During each interval there is no transaction. All dividends of risky security are re-invested continually in the risky security, and all interest income is re-invested continually in the riskless security.

The investor chooses instants of time at which to process information and make consumption and investment decisions. The investor receives information via controllable filtration

$$
\mathbf{H}=\left\{\mathcal{H}_{t}: t \geq 0\right\} \quad \text { with } \quad \mathcal{H}_{t}=\mathcal{F}_{t}, \quad t \in\left[\tau_{n}, \tau_{n+1}\right)
$$

where $\tau_{n}$ is a $\mathcal{H}_{\tau_{n-1}}$-measurable stopping time at which the $\mathrm{n}$-th transaction occurs. The filtration $\mathbf{H}$ is controllable in the sense that the investor is allowed to choose any sequence $\tau=\left\{\tau_{n}: n=1,2,3, \ldots\right\}$ of such transaction times with $\tau_{1} \equiv 0$. Let $T=\left\{T_{n}=\tau_{n+1}-\tau_{n}: n=1,2,3, \ldots\right\}$ denotes the corresponding sequence of transaction intervals. Finding an optimal stopping policy $\tau$ is clearly equivalent to finding an optimal transaction interval policy $T$. 


\subsection{The Model}

\subsubsection{Preferences}

Let the consumption space $\mathcal{C}$ for the investor consists of positive $\mathbf{H}$-adapted consumption processes $\mathbf{C}=\left\{C_{t}: t \geq 0\right\}$ satisfying $\int_{0}^{t} C_{s} d s<\infty$ almost surely for all $t \geq 0$, and

$$
E\left[\int_{0}^{\infty} e^{-\delta t} u\left(C_{t}\right) d t\right]<\infty
$$

where $E$ denotes the expected value function, with respect to $P, T_{f}<\infty$ is the final time, $\delta$ is a strictly positive scalar discount factor and the utility function $u$, is one of the HARA (hyperbolic absolute risk-aversion) type function, as defined in [19]. We take $u$ as given by

$$
u(C)=\frac{1}{\gamma} C^{\gamma}, \quad 0<\gamma<1
$$

\subsubsection{Feasible policies}

Let $\tau=\left\{\tau_{n}: n=1,2,3, \ldots\right\}$ be sequence of transaction times with $\tau_{1} \equiv 0$. Let $T=\left\{T_{n}=\tau_{n+1}-\tau_{n}, n=1,2,3, \ldots\right\}$ be the sequence of corresponding transaction intervals. Let $W=\left\{W_{\tau_{n}}: n=1,2,3, \ldots\right\}$ be the sequence of money withdrawal processes, and $V=\left\{V_{\tau_{n}}: n=1,2,3, \ldots\right\}$ be the sequence of investment for the risky security.

Let $\mathcal{T}$ denote the space of sequences of strictly positive transaction intervals, $\mathcal{W}$ the space of positive $\mathbf{H}$-adapted money withdrawal processes, and $\mathcal{V}$ the space of $\mathbf{H}$-adapted investment processes for the risky security. Let $\mathcal{U}=\mathcal{T} \times \mathcal{W} \times \mathcal{V} \times \mathcal{C}$. 
Definition 2.6 A budget policy is a quadruplet $(T, W, V, C) \in \mathcal{U}$.

We characterize budget feasible policies as follows. Let $\mathcal{U}$ denotes a class of budget policies. Given a policy $(T, W, V, C) \in \mathcal{U}$, then the money holding at any time $\mathrm{t}$ is defined by

$$
M_{t}=\sum_{\left\{n: \tau_{n} \leq t\right\}}\left[W_{\tau_{n}}-\Psi\left(W_{\tau_{n}}\right)\right]-\int_{0}^{t} C_{s} d s,
$$

where $\Psi$ is the transaction costs function as defined in Section 2.2.

Let $X_{\tau_{n}}$ denotes the total wealth invested in the securities at time $\tau_{n}$, before the nth transaction. Let $W_{\tau_{n}}$ denotes the amount of money withdrawn at time $\tau_{n}$ from the total wealth $X_{\tau_{n}}$, and $V_{\tau_{n}}$ denotes the market value of the investment in the risky security chosen at time $\tau_{n}$. After an amount $W_{\tau_{n}}$ is withdrawn from the total wealth $X_{\tau_{n}}$, and a fraction $\varepsilon$ of the remainder, is paid as management fees, then the wealth left for re-investment is $Z_{\tau_{n}}=(1-\varepsilon)\left[X_{\tau_{n}}-W_{\tau_{n}}\right]$. Of this amount, $V_{\tau_{n}}$ is invested in the risky security with a per-dollar payback of $\Gamma_{n+1}$ at the next transaction date, including continually re-invested dividends. And the remainder, $Z_{\tau_{n}}-V_{\tau_{n}}$, is invested in the riskless security at the continuously compounding interest rate $r>0$.

The investor's total wealth invested at the time of the $(n+1)$ th transaction is therefore, for $n=1,2,3, \ldots$,

$$
X_{\tau_{n+1}}=(1-\varepsilon)\left[X_{\tau_{n}}-W_{\tau_{n}}\right] e^{r T_{n}}+V_{\tau_{n}}\left[\Gamma_{n+1}-e^{r T_{n}}\right] .
$$

According to the equation( 2.2) and the Itô's formula, ${ }^{2}$ the return of the

\footnotetext{
${ }^{2}$ Details may be found in Karatzas and Shreve [12], or Protter [22]
} 
risky investment $\Gamma$ satisfies

$$
\Gamma_{n+1}=\exp \left[\left(\alpha-\frac{1}{2} \sigma^{2}\right) T_{n}+\sigma\left(B_{\tau_{n+1}}-B_{\tau_{n}}\right)\right]
$$

Since $M_{0}=0$, then $X_{0}$ is considered as the initial wealth endowment for the investor.

Definition 2.7 The budget policy $(T, W, V, C) \in \mathcal{U}$ is budget feasible policy if the associated money process $M$ of (2.5) and invested wealth process $X$ of ( 2.6) are non-negative.

\subsection{The Problem}

The discrete-continuous-time formulation of consumption and investment problem for the investor is summarized in the following definition.

Definition 2.8 The optimal control problem for the investor is given by

$$
U\left(X_{0}\right) \equiv \max _{(T, W, V, C) \in \mathcal{U}} E\left[\int_{0}^{\infty} e^{-\delta t} u\left(C_{t}\right) d t\right]
$$

subject to, for $n=1,2,3, \ldots$,

$$
X_{\tau_{n+1}}=(1-\varepsilon)\left[X_{\tau_{n}}-W_{\tau_{n}}\right] e^{r T_{n}}+V_{\tau_{n}}\left[\Gamma_{n+1}-e^{r T_{n}}\right]
$$

and $M_{t} \geq 0$, and $X_{\tau_{n+1}} \geq 0$. 


\section{Chapter 3}

\section{Portfolio Selection with Bullet Transaction Costs}

\subsection{Introduction}

Chapter 3 is concerned with consumption and investment strategy for an investor who seeks to maximize the expected utility of consumption. The investor has available a riskless asset paying fixed interest rate and a risky asset with logarithmic Brownian motion price fluctuations. The objective is to maximize the expected discounted utility of consumption. The investor observes his/her current wealth and makes transaction at stopping times $\tau_{1}, \tau_{2}, \tau_{3}, \ldots$. The decision to transact can be made at any time based on all current information. For every transaction, the investor is charged a fraction of the current wealth as a management fee plus transaction costs which is fixed for every transaction, regardless of the amount of wealth transacted. 
The problem faced by the investor, as formulated in Chapter 2, is in a discrete-continuous time optimal control problem form. The optimal control problem for the investor is to choose optimal policy $(T, W, V, C)$ in a set of feasible policies $\mathcal{U}$, such that the value function is maximized. The main task of Chapter 3 is to find an optimal solution to that optimal control problem. The task is accomplished by solving the problem in two steps. In the first step, a deterministic continuous-time optimal control problem for consumption is solved, for a given $(T, W, V)$. In the second step, equipped with the optimal value function from the first step, a stochastic discrete-time optimal control problem is solved. The existence of an optimal consumption and investment selection is given.

The rest of Chapter 3 is organized as follows. Section 3.2 states the problem which is formulated in Chapter 2. In Section 3.3, we show that it is not optimal for the investor to withdraw more money than the amount needed for consumption. For a given $(T, W, V)$, the optimal consumption for the investor is solved. Section 3.4 is concerned with the derivation of optimal money withdrawals process and investment strategy for the risky security. This is done by deriving the optimal value function for fixed interval $T_{n}$ for all $\mathrm{n}$. This is one of the main features of Chapter 3 as well as this thesis. In Section 3.5, with optimal $(W, V, C)$ in hand, an equation satisfied by the transaction intervals is derived. Each optimal transaction interval infact satisfies a nonlinear equation which is not independent of total wealth at the beginning of that interval. A solution to that equation exists and is optimal. In addition, in Section 3.6 we confirm the result of [7] that when $b=0$, then the optimal transaction intervals are equal. 


\subsection{Statement of the Problem}

The model for the investor is as in Chapter 2, with fixed transaction costs. That is, the transaction costs function $\Psi$ takes of the form $\Psi\left(W_{\tau_{n}}\right)=b$, with $b \geq 0$. Then the money holding at any time $t$ is given by

$$
M_{t}=\sum_{\left\{n: \tau_{n} \leq t\right\}}\left[W_{\tau_{n}}-b\right]-\int_{0}^{t} C_{s} d s .
$$

Definition 3.1 Let $\mathcal{U}$ be the set of all budget feasible policies as defined in Chapter 2. The optimal control problem for the investor is to maximize

$$
U\left(X_{0}\right) \equiv \max _{(T, W, V, C) \in \mathcal{U}} E\left[\int_{0}^{\infty} e^{-\delta t} u\left(C_{t}\right) d t\right]
$$

subject to, for $n=1,2,3, \ldots$,

$$
X_{\tau_{n+1}}=(1-\varepsilon)\left[X_{\tau_{n}}-W_{\tau_{n}}\right] e^{r T_{n}}+V_{\tau_{n}}\left[\Gamma_{n+1}-e^{r T_{n}}\right],
$$

with $M_{t} \geq 0$, and $X_{\tau_{n+1}} \geq 0$.

\subsection{Consumption Strategy}

Section 3.3 is concerned with the derivation of optimal consumption strategy for the investor. It is assumed in Chapter 2 that only money is available to the investor as a medium of exchange and numeraire in the economy. Only money is exchangeable for consumption. It is also assumed that money cannot be borrowed, it can only be acquired by selling the securities, and it is put in the purse $M$. Because there exists a riskless security with a positive interest rate in the economy, there is no investment demand for money. Duffie 
and Sun in [7] argued that it will not be optimal for the investor to withdraw more money than the amount needed for financing consumption before the next transaction.

The following result is similar to those in [7].

Lemma 3.1 Let the value function $U$ be defined as in (3.2), and the transaction costs function $\Psi\left(W_{\tau_{n}}\right)=b, \quad b \geq 0$. Then the optimal policy $(T, W, V, C)$ must satisfy for all $n=1,2,3, \ldots$

$$
\int_{\tau_{n}}^{\tau_{n+1}} C_{t} d t=W_{\tau_{n}}-b
$$

Proof : The proof is similar to those in [7]. Let $(T, W, V, C)$ be an optimal policy. Suppose that there exists an interval $T_{j}=\tau_{j+1}-\tau_{j}$ such that $d>0$ where $d$ is defined by

$$
d=W_{\tau_{j}}-b-\int_{\tau_{j}}^{\tau_{j+1}} C_{t} d t
$$

Because there exists a riskless security with a positive interest rate, then the investor will be better off if the amount $d$ is invested in the riskless security during the interval $T_{j}$, and the interest income $d\left(e^{r T_{j}}-1\right)$ is consumed in the next interval. In other words, the optimal policy $(T, W, V, C)$ is dominated by a feasible policy $(T, \bar{W}, V, \bar{C})$, which is defined by

$$
\begin{gathered}
\bar{W}_{\tau_{j}}=b+\int_{\tau_{j}}^{\tau_{j+1}} C_{t} d t, \\
\bar{W}_{\tau_{j+1}}=W_{\tau_{j+1}}+d e^{r T_{j}}>W_{\tau_{j+1}}, \\
\bar{C}_{t}=C_{t}+\frac{1}{T_{j+1}} d\left(e^{r T_{j}}-1\right)>C_{t}, \quad t \in\left[\tau_{j+1}, \tau_{j+2}\right), \\
\bar{C}_{t}=C_{t}, \quad \bar{W}_{\tau_{j}}=W_{\tau_{j}}, \quad \text { otherwise. }
\end{gathered}
$$


This contradicts with the fact that $(T, W, V, C$, ) is optimal. Therefore, for all $n$,

$$
\int_{\tau_{n}}^{\tau_{n+1}} C_{t} d t \geq W_{\tau_{n}}-b
$$

On the other hand, all of expenditures must be financed from the stock of money. Therefore, for all $n$,

$$
\sum_{i}^{n} \int_{\tau_{i}}^{\tau_{i+1}} C_{t} d t \geq \sum_{i}^{n}\left[W_{\tau_{i}}-b\right]
$$

Therefore, for all $n$, an optimal policy $(T, W, V, C)$ must satisfy

$$
\int_{\tau_{n}}^{\tau_{n+1}} C_{t} d t=W_{\tau_{n}}-b
$$

Hence, the proof of Lemma 3.1 has been completed

Corollary 3.1 By the definition of money holding $M_{t}$ of (3.1), then

$$
M_{\tau_{n}}=W_{\tau_{n}}-b, \quad n=1,2,3, \ldots
$$

Therefore, the optimal control problem (3.2)-( 3.3) is equivalent to the optimal control problem :

$$
U\left(X_{0}\right)=\max _{(T, W, V, C) \in \mathcal{U}} E\left[\int_{0}^{\infty} e^{-\delta t} u\left(C_{t}\right) d t\right]
$$

subject to, for $n=1,2,3, \ldots$,

$$
\begin{gathered}
\int_{\tau_{n}}^{\tau_{n+1}} C_{t} d t=W_{\tau_{n}}-b, \\
X_{\tau_{n+1}}=(1-\varepsilon)\left[X_{\tau_{n}}-W_{\tau_{n}}\right] e^{r T_{n}}+V_{\tau_{n}}\left[\Gamma_{n+1}-e^{r T_{n}}\right] \geq 0 .
\end{gathered}
$$

The term under the expectation in ( 3.5) may be re-written as :

$$
\int_{0}^{\infty} e^{-\delta t} u\left(C_{t}\right) d t=\sum_{n=1}^{\infty} e^{-\delta \tau_{n}} \int_{\tau_{n}}^{\tau_{n+1}} e^{-\delta\left(t-\tau_{n}\right)} u\left(C_{t}\right) d t
$$


Therefore, the equation ( 3.5$)$ may be re-written as :

$$
U\left(X_{0}\right)=E\left[\sum_{n=1}^{\infty} e^{-\delta \tau_{n}} \int_{\tau_{n}}^{\tau_{n+1}} e^{-\delta\left(t-\tau_{n}\right)} u\left(C_{t}\right) d t\right]
$$

Hence, the control problem ( 3.5$)$ - (3.7) will be solved in two steps. In the first step, the control problem for consumption between transaction intervals is solved for any given budget feasible $(\mathrm{T}, \mathrm{W}, \mathrm{V})$. This control problem is a deterministic continuous-time control problem, because the consumption $\mathrm{C}$ is adapted to the filtration $\mathbf{H}$. Let the objective function for this problem be denoted by $J$. In the second step, the investor chooses a budget feasible $(\mathrm{T}, \mathrm{W}, \mathrm{V})$ to maximize $E\left[\sum_{n=1}^{\infty} e^{-\delta \tau_{n}} J\left(T_{n}, M_{\tau_{n}}\right)\right]$. This is similar to a stochastic discrete-time control problem except that the sequence $\mathrm{T}$ of transaction intervals is controllable.

Consider an investor with an initial money endowment $\mathrm{Z}$ and time-horizon $\mathrm{t}$. The deterministic control problem for the investor is to maximize the objective function

$$
J(t, Z) \equiv \int_{0}^{t} \exp (-\delta s) u\left(C_{s}\right) d s
$$

over $\left\{C_{s}: 0 \leq s \leq t\right\}$, subject to :

$$
\int_{0}^{t} C_{s} d s \leq Z .
$$

Lemma 3.2 The optimal value function $J$ for the deterministic control problem (3.8) - (3.9) satisfies

$$
J(t, Z)=\left(\frac{1-\gamma}{\delta}\right)^{1-\gamma}\left[1-\exp \left(-\frac{\delta}{1-\gamma} t\right)\right]^{1-\gamma} \frac{1}{\gamma} Z^{\gamma} .
$$

Proof : The above problem falls in the category of isoperimetric problem in the calculus of variations (see [1]). Hence, the above problem can be 
solved by a Lagrange multiplier technique. Since the problem is to maximize consumption, then the consumption can always be increased such that the left hand side of ( 3.9$)$ is equal to the right hand side of ( 3.9). By appending ( 3.9) into ( 3.8), then a Lagrange multiplier gives

$$
L=\int_{0}^{t}\left[\exp (-\delta s) u\left(C_{s}\right)-\lambda C_{s}\right] d s+\lambda Z,
$$

where $\lambda$ is a Lagrange mulptiplier. A necessary condition for $C$ to maximize the augmented integrand of $\mathrm{L}$ is that it satisfies the Euler equation

$$
\exp (-\delta \tau) u^{\prime}\left(C_{\tau}\right)=\lambda
$$

Therefore, the Lemma is proved by solving the following problem :

$$
\exp (-\delta \tau) u^{\prime}\left(C_{\tau}\right)=\lambda
$$

where $u^{\prime}$ denotes the first derivative of the utility function $u, C_{\tau}$ denotes the optimal consumption at time $\tau$. From the definition of the utility function $u$ in (2.4), then its first derivative $u^{\prime}$ is given by

$$
u^{\prime}\left(C_{\tau}\right)=C_{\tau}^{\gamma-1}, \quad 0<\gamma<1
$$

Then by substituting this derivative into ( 3.11 ) yields

$$
C_{\tau}=[\lambda \exp (\delta \tau)]^{-1 /(1-\gamma)}
$$

Since the control problem is to maximize the utility function, then $C_{\tau}$ optimal must satisfy

$$
Z=\int_{0}^{t} C_{\tau} d \tau
$$


Use the last equation and equation ( 3.12) to get

$$
C_{\tau}=\left(\frac{1-\gamma}{\delta}\right)^{-1}\left[1-\exp \left(-\frac{\delta}{1-\gamma} t\right)\right]^{-1} Z \exp \left(-\frac{\delta}{1-\gamma} \tau\right)
$$

Finally, by insertion of ( 3.13 ) into ( 3.8$)$ results in

$$
J(t, Z)=\left(\frac{1-\gamma}{\delta}\right)^{1-\gamma}\left[1-\exp \left(-\frac{\delta}{1-\gamma} t\right)\right]^{1-\gamma} \frac{1}{\gamma} Z^{\gamma}
$$

Since $0<\gamma<1$, then without loss of the generality, the term $\left(\frac{1-\gamma}{\delta}\right)^{1-\gamma}$ will be left out in future discussions. Therefore, by Corollary 3.1,

$$
J\left(T_{n}, M_{\tau_{n}}\right)=\left[1-\exp \left(-\frac{\delta}{1-\gamma} T_{n}\right)\right]^{1-\gamma} \frac{1}{\gamma}\left(W_{\tau_{n}}-b\right)^{\gamma} .
$$

Now let $Q_{n}=1-\exp \left(-\frac{\delta}{\nu} T_{n}\right)$, with $\nu=1-\gamma$. Then the modified optimal control problem is given by

$$
U\left(X_{0}\right)=\max _{\{T \in \mathcal{T}, W \in \mathcal{W}, V \in \mathcal{V}\}} E\left[\sum_{n=1}^{\infty} e^{-\delta \tau_{n}} Q_{n}^{\nu} \frac{1}{\gamma}\left(W_{\tau_{n}}-b\right)^{\gamma}\right]
$$

subject to, for $n=1,2,3, \ldots$,

$$
X_{\tau_{n+1}}=(1-\varepsilon)\left[X_{\tau_{n}}-W_{\tau_{n}}\right] e^{r T_{n}}+V_{\tau_{n}}\left[\Gamma_{n+1}-e^{r T_{n}}\right] \geq 0,
$$

where $T_{n}=\tau_{n+1}-\tau_{n}$.

The application of Bellman principle on $U$, results in

$$
U\left(X_{\tau_{n}}\right)=\max _{\left\{T_{n}, W_{\tau_{n}}, V_{\tau_{n}}\right\}}\left\{Q_{n}^{\nu} \frac{1}{\gamma}\left(W_{\tau_{n}}-b\right)^{\gamma}+e^{-\delta T_{n}} E\left[U\left(X_{\tau_{n+1}}\right) \mid \mathcal{H}_{\tau_{n}}\right]\right\},
$$

subject to, for $n=1,2,3, \ldots$,

$$
X_{\tau_{n+1}}=(1-\varepsilon)\left[X_{\tau_{n}}-W_{\tau_{n}}\right] e^{r T_{n}}+V_{\tau_{n}}\left[\Gamma_{n+1}-e^{r T_{n}}\right] .
$$


The following result is proved in [7] but is given for completeness.

Lemma 3.3 Let $\bar{Q}(n)=\left[1-\exp \left(-\frac{\delta}{\nu} T_{n}\right)\right]^{\nu}$. Suppose that $f$ is a realvalued function on $[0, \infty)$ satisfying the two conditions :

(i) For all $n=1,2,3, \ldots$,

$$
f\left(X_{\tau_{n}}\right)=\max _{\left(T_{n}, W_{\tau_{n}}, V_{\tau_{n}}\right)}\left\{\bar{Q}(n) \frac{1}{\gamma}\left(W_{\tau_{n}}-b\right)^{\gamma}+E\left[e^{-\delta T_{n}} f\left(X_{\tau_{n+1}}\right)\right] \mid \mathcal{H}_{\tau_{n}}\right\} .
$$

(ii) For any feasible policy,

$$
\lim _{n \rightarrow \infty} E\left[e^{-\delta \tau_{n}} f\left(X_{\tau_{n}}\right)\right]=0 .
$$

If $\left(T^{*}, W^{*}, V^{*}\right)$ achieves the maximum in (3.18) for all $n$ then $f$ is the value function for the control problem (3.14), and $\left(T^{*}, W^{*}, V^{*}\right)$ is an optimal policy.

Proof : Let $n=1$, to begin with, that is $\tau_{1}=0$. Then

$$
\begin{aligned}
f\left(X_{0}\right) & =\max _{\left(T_{1}, W_{0}, V_{0}\right)}\left\{\bar{Q}(1) \frac{1}{\gamma}\left(W_{0}-b\right)^{\gamma}+E\left[e^{-\delta T_{1}} f\left(X_{\tau_{2}}\right)\right]\right\} \\
& \geq \bar{Q}(1) \frac{1}{\gamma}\left(W_{0}-b\right)^{\gamma}+e^{-\delta T_{1}} E\left[f\left(X_{\tau_{2}}\right)\right]
\end{aligned}
$$

for any feasible $T_{1}, W_{0}, V_{0}$. By induction, for any $(T, W, V) \in \mathbf{T} \times \mathbf{W} \times \mathbf{V}$ then

$$
f\left(X_{0}\right) \geq E\left[\sum_{i=1}^{n} e^{-\delta \tau_{i}} \bar{Q}(i) \frac{1}{\gamma}\left(W_{\tau_{i}}-b\right)^{\gamma}+e^{-\delta \tau_{n+1}} f\left(X_{\tau_{n+1}}\right)\right] .
$$

Let $n \rightarrow \infty$, it follows by condition (ii) of Lemma 3.3 that

$$
f\left(X_{0}\right) \geq E\left[\sum_{n=1}^{\infty} e^{-\delta \tau_{n}} \bar{Q}(n) \frac{1}{\gamma}\left(W_{\tau_{n}}-b\right)^{\gamma}\right] .
$$


This holds for an arbitrary feasible policy $(T, V, W)$. Hence,

$$
f\left(X_{0}\right) \geq U\left(X_{0}\right)
$$

On the other hand, $U\left(X_{0}\right) \geq f\left(X_{0}\right)$ by the definition of $U\left(X_{0}\right)$. Henceforth, $f\left(X_{0}\right)=U\left(X_{0}\right)$, and consequently $\left(T^{*}, W^{*}, V^{*}\right)$ is optimal 


\subsection{Investment Strategies}

Section 3.4 is mainly concerned with the derivation of optimal solution to the stochastic optimal control problem ( 3.14) - (3.15). The optimal value function and the optimal withdrawal process as well as the optimal investment decisions are derived. The proceeding of Section 3.4 begins with discussions of some preliminary results which will be usefull in future discussions.

The following result is similar to those in [11], and its proof is omitted.

Lemma 3.4 Let $u, \Gamma_{n+1}, \quad r$, and $T_{n}$ be defined as in Chapter 2. Then the function

$$
f(\pi) \equiv E\left[u\left(e^{r T_{n}}+\pi\left(\Gamma_{n+1}-e^{r T_{n}}\right)\right)\right]
$$

subject to the constraints $\pi \geq 0$, and

$$
P\left\{e^{r T_{n}}+\pi\left(\Gamma_{n+1}-e^{r T_{n}}\right) \geq 0\right\}=1,
$$

has a finite maximum.

Lemma 3.5 Let $\pi \geq 0$, then $P\left\{e^{r T_{n}}+\pi\left(\Gamma_{n+1}-e^{r T_{n}}\right) \geq 0\right\}=1$ holds if and only if $\pi \leq 1$.

Remark 3.1 Below maximizing with $0 \leq \pi \leq 1$ is equivalent to maximizing with $\pi \geq 0$ and (3.21).

Proof : Let $P\left\{e^{r T_{n}}+\pi\left(\Gamma_{n+1}-e^{r T_{n}}\right) \geq 0\right\}=1$. Then it will be shown that $\pi \leq 1$.

If $\pi>1$, it will be shown that

$$
P\left\{e^{r T_{n}}+\pi\left(\Gamma_{n+1}-e^{r T_{n}}\right) \geq 0\right\}<1 .
$$


Note that

$$
e^{r T_{n}}+\pi\left(\Gamma_{n+1}-e^{r T_{n}}\right) \geq 0 \Longleftrightarrow \Gamma_{n+1} \geq e^{r T_{n}}\left(1-\frac{1}{\pi}\right)
$$

Let $\lambda=\alpha-\frac{1}{2} \sigma^{2}$. Then

$$
\begin{aligned}
P\left\{\Gamma_{n+1} \geq e^{r T_{n}}\left(1-\frac{1}{\pi}\right)\right\} & =P\left\{\lambda T_{n}+\sigma B_{T_{n}}>r T_{n}+\log \left(1-\frac{1}{\pi}\right)\right\} \\
& =P\left\{B_{T_{n}}>\frac{1}{\sigma}(r-\lambda) T_{n}+\frac{1}{\sigma} \log \left(1-\frac{1}{\pi}\right)\right\} .
\end{aligned}
$$

Let $l=\frac{1}{\sigma} \log (1-1 / \pi)$, and $m=\frac{1}{\sigma}\left(r-\alpha+\frac{1}{2} \sigma^{2}\right)$. Then,

$$
\begin{aligned}
P\left\{B_{T_{n}} \geq l+m T_{n}\right\} & =\int_{l+m T_{n}}^{\infty} e^{-\frac{\zeta^{2}}{2} T_{n}} \frac{1}{\sqrt{2 \pi T_{n}}} d \zeta \\
& =\int_{\frac{l}{\sqrt{T_{n}}}+m \sqrt{T_{n}}}^{\infty} e^{-\frac{y^{2}}{2}} \frac{1}{\sqrt{2 \pi}} d y \\
& <1 .
\end{aligned}
$$

On the other hand, if $\pi \leq 1$, then

$$
e^{r T_{n}}+\pi\left(\Gamma_{n+1}-e^{r T_{n}}\right)=(1-\pi) e^{r T_{n}}+\pi \Gamma_{n+1} \geq 0 .
$$


Lemma 3.6 Let $\delta>\max (\gamma \alpha, \gamma r)$, and let $\Omega_{n}$ be defined by

$$
\begin{aligned}
\Omega_{n} & \equiv E\left(\left[e^{r T_{n}}+\pi_{n}\left(\Gamma_{n+1}-e^{r T_{n}}\right)\right]^{\gamma}\right) \\
& \equiv \sup _{\{0 \leq \pi \leq 1\}} E\left(\left[e^{r T_{n}}+\pi\left(\Gamma_{n+1}-e^{r T_{n}}\right)\right]^{\gamma}\right) .
\end{aligned}
$$

Then $R_{n}=(1-\varepsilon)^{\gamma} e^{-\delta T_{n}} \Omega_{n}$ is such that $R_{n} \leq(1-\varepsilon)^{\gamma}$.

Proof : Since $0<\varepsilon<1$ and $\pi_{n} \in[0,1]$, then

$$
\begin{aligned}
R_{n} & =(1-\varepsilon)^{\gamma} e^{-\delta T_{n}} \Omega_{n} \\
& =(1-\varepsilon)^{\gamma} e^{-\delta T_{n}} E\left(\left[e^{r T_{n}}+\pi_{n}\left(\Gamma_{n+1}-e^{r T_{n}}\right)\right]^{\gamma}\right) \\
& =(1-\varepsilon)^{\gamma} e^{-\delta T_{n}}\left[e^{r T_{n}}+\pi_{n}\left(E\left(\Gamma_{n+1}\right)-e^{r T_{n}}\right)\right]^{\gamma} \\
& =(1-\varepsilon)^{\gamma} e^{-\delta T_{n}}\left[e^{r T_{n}}+\pi_{n}\left(e^{\alpha T_{n}}-e^{r T_{n}}\right)\right]^{\gamma} \\
& =(1-\varepsilon)^{\gamma} e^{-\delta T_{n}}\left[e^{r T_{n}}+1_{\{\alpha \geq r\}}\left(e^{\alpha T_{n}}-e^{r T_{n}}\right)\right]^{\gamma} \\
& \leq(1-\varepsilon)^{\gamma} e^{-\delta T_{n}} e^{\gamma r T_{n}+\gamma 1_{\{\alpha \geq r\}}(\alpha-r) T_{n}} \\
& \leq(1-\varepsilon)^{\gamma} e^{-\delta T_{n}} e^{\max (\gamma \alpha, \gamma r) T_{n}} .
\end{aligned}
$$

Since $\delta>\max (\gamma \alpha, \gamma r)$ by assumption, then $e^{-\delta T_{n}} e^{\max (\gamma \alpha, \gamma r) T_{n}}<1$. Therefore, $\quad R_{n}=(1-\varepsilon)^{\gamma} e^{-\delta T_{n}} \Omega_{n} \leq(1-\varepsilon)^{\gamma}$. 
Theorem 3.1 Let $T_{n}$ be fixed for $n=1,2,3, \ldots$. Then the optimal value function and unique solution to problem (3.16)- (3.17), is given by

$$
U\left(X_{\tau_{n}}\right)=Q_{n}^{\nu} A_{n}^{-\nu} \frac{1}{\gamma}\left(X_{\tau_{n}}-Y_{n}\right)^{\gamma}
$$

with the optimal withdrawal and investment strategies are given by

$$
\begin{aligned}
W_{\tau_{n}} & =A_{n}\left(X_{\tau_{n}}-Y_{n}\right)+b \\
V_{\tau_{n}} & =(1-\varepsilon)\left(1-A_{n}\right)\left(X_{\tau_{n}}-Y_{n}\right) \pi_{n}
\end{aligned}
$$

respectively, and where $A_{n}$, and $Y_{n}$ are given by

$$
\begin{aligned}
A_{n} & =\frac{A_{n+1} Q_{n}}{A_{n+1} Q_{n}+Q_{n+1} R_{n}^{1 / \nu}} \\
Y_{n} & =b+(1-\varepsilon)^{-1} e^{-r T_{n}} Y_{n+1}
\end{aligned}
$$

respectively, with $R_{n}=(1-\varepsilon)^{\gamma} e^{-\delta T_{n}} \Omega_{n}$, and where $\Omega_{n}$ and $\pi_{n}$ are defined by the optimization problem

$$
\begin{aligned}
\Omega_{n} & \equiv E\left(\left[e^{r T_{n}}+\pi_{n}\left(\Gamma_{n+1}-e^{r T_{n}}\right)\right]^{\gamma}\right) \\
& \equiv \sup _{\{0 \leq \pi \leq 1\}} E\left(\left[e^{r T_{n}}+\pi\left(\Gamma_{n+1}-e^{r T_{n}}\right)\right]^{\gamma}\right) .
\end{aligned}
$$

Remark 3.2 Equation (3.23) and $C \geq 0$ imply that $W_{\tau_{n}}-b \geq 0$, and hence $X_{\tau_{n}} \geq Y_{n}$. 
Proof of Theorem : The idea of the proof is similar to those in [11]. Let the right-hand side of (3.16) be denoted by $S\left(X_{\tau_{n}}\right)$ upon inserting (3.22) for $U\left(X_{\tau_{n}}\right)$. Then we have

$$
\begin{aligned}
S\left(X_{\tau_{n}}\right) & =\max _{\left\{W_{\tau_{n}}, V_{\tau_{n}}\right\}}\left\{Q_{n}^{\nu} \frac{1}{\gamma}\left(W_{\tau_{n}}-b\right)^{\gamma}\right. \\
& \left.+Q_{n+1}^{\nu} A_{n+1}^{-\nu} e^{-\delta T_{n}} E\left[\frac{1}{\gamma}\left(X_{\tau_{n+1}}-Y_{n+1}\right)^{\gamma} \mid \mathcal{H}_{\tau_{n}}\right]\right\} .
\end{aligned}
$$

Furthermore, let $Y_{n}$ be defined by recurrence relationship

$$
Y_{n}=b+(1-\varepsilon)^{-1} e^{-r T_{n}} Y_{n+1}
$$

with $b, \varepsilon, r$ are as before. Then the total wealth process $X_{\tau_{n+1}}$ of $(3.17)$ may be written as

$$
\begin{aligned}
X_{\tau_{n+1}} & =(1-\varepsilon)\left[X_{\tau_{n}}-Y_{n}-\left(W_{\tau_{n}}-b\right)\right] e^{r T_{n}} \\
& +V_{\tau_{n}}\left(\Gamma_{n+1}-e^{r T_{n}}\right)+Y_{n+1} .
\end{aligned}
$$

This implies that $S\left(X_{\tau_{n}}\right)$ may be written as

$$
\begin{aligned}
S\left(X_{\tau_{n}}\right) & =\max _{\left\{W_{\tau_{n}}, V_{\tau_{n}}\right\}}\left\{Q_{n}^{\nu} \frac{1}{\gamma}\left(W_{\tau_{n}}-b\right)^{\gamma}+A_{n+1}^{-\nu} Q_{n+1}^{\nu} e^{-\delta T_{n}}\right. \\
& \times E\left[\frac { 1 } { \gamma } \left((1-\varepsilon)\left[X_{\tau_{n}}-Y_{n}-\left(W_{\tau_{n}}-b\right)\right] e^{r T_{n}}\right.\right. \\
& \left.\left.\left.+V_{\tau_{n}}\left(\Gamma_{n+1}-e^{r T_{n}}\right)\right)^{\gamma}\right]\right\},
\end{aligned}
$$

subject to :

$$
\begin{gathered}
W_{\tau_{n}}-b \geq 0 \\
P\left\{X_{\tau_{n+1}}-Y_{n+1} \geq 0\right\}=1, \\
V_{\tau_{n}} \geq 0 .
\end{gathered}
$$


To prevent the problem being trivial, the following assumption is imposed :

$$
P\left\{\theta\left(\Gamma_{n+1}-e^{r T_{n}}\right)<0\right\}>0, \text { for some } \theta>0 .
$$

Notice that, for $X_{\tau_{n}}-Y_{n}-\left(W_{\tau_{n}}-b\right)>0$, by re-arrangement, the total wealth process $X_{\tau_{n+1}}$ of (3.29) may be re-written as

$$
\begin{aligned}
X_{\tau_{n+1}} & =(1-\varepsilon)\left[X_{\tau_{n}}-Y_{n}-\left(W_{\tau_{n}}-b\right)\right] \\
& \times\left[e^{r T_{n}}+I_{n}\left(\Gamma_{n+1}-e^{r T_{n}}\right)\right]+Y_{n+1},
\end{aligned}
$$

with $I_{n}$ is given by

$$
I_{n}=\frac{V_{\tau_{n}}}{(1-\varepsilon)\left[X_{\tau_{n}}-Y_{n}-\left(W_{\tau_{n}}-b\right)\right]} .
$$

This implies that $P\left\{X_{\tau_{n+1}}-Y_{n+1} \geq 0\right\}=1$, can only occur when either

$$
X_{\tau_{n}}-Y_{n}-\left(W_{\tau_{n}}-b\right)=0, \text { and } V_{\tau_{n}}=0
$$

or,

$$
X_{\tau_{n}}-Y_{n}-\left(W_{\tau_{n}}-b\right)>0
$$

and

$$
P\left\{e^{r T_{n}}+I_{n}\left(\Gamma_{n+1}-e^{r T_{n}}\right) \geq 0\right\}=1 .
$$

Under feasibility with respect to (3.32), then

$$
S\left(X_{\tau_{n}}\right)=\max \left\{Q_{n}^{\nu} \frac{1}{\gamma}\left(W_{\tau_{n}}-b\right)^{\gamma}, \bar{S}\left(X_{\tau_{n}}\right)\right\}
$$

where

$$
\begin{aligned}
\bar{S}\left(X_{\tau_{n}}\right) & =\sup _{\left\{W_{\tau_{n}}, V_{\tau_{n}}\right\}}\left\{Q_{n}^{\nu} \frac{1}{\gamma}\left[W_{\tau_{n}}-b\right]^{\gamma}\right. \\
& +Q_{n+1}^{\nu} A_{n+1}^{-\nu}(1-\varepsilon)^{\gamma} e^{-\delta T_{n}}\left[X_{\tau_{n}}-Y_{n}-\left(W_{\tau_{n}}-b\right)\right]^{\gamma} \\
& \left.\times E\left[\frac{1}{\gamma}\left(e^{r T_{n}}+I_{n}\left(\Gamma_{n+1}-e^{r T_{n}}\right)\right)^{\gamma}\right]\right\},
\end{aligned}
$$


subject to $(3.31),(3.37),(3.38)$ and $I_{n} \geq 0$, since this is equivalent to (3.33) in view of (3.38).

The expectation factor in (3.40) may be re-written as $f\left(I_{n}\right)$, where $f$ is defined by

$$
f(\pi)=E\left[u\left(\exp \left(r T_{n}\right)+\pi\left(\Gamma_{n+1}-\exp \left(r T_{n}\right)\right)\right)\right],
$$

where utility function $u$ is given by $u(C)=\frac{1}{\gamma} C^{\gamma}, \quad \gamma \in(0,1)$. Therefore

$$
\begin{aligned}
\bar{S}\left(X_{\tau_{n}}\right) & =\sup _{\left\{W_{\tau_{n}}, V_{\tau_{n}}\right\}}\left\{Q_{n}^{\nu} \frac{1}{\gamma}\left[W_{\tau_{n}}-b\right]^{\gamma}+Q_{n+1}^{\nu} A_{n+1}^{-\nu}\right. \\
& \left.\times(1-\varepsilon)^{\gamma} e^{-\delta T_{n}}\left[X_{\tau_{n}}-Y_{n}-\left(W_{\tau_{n}}-b\right)\right]^{\gamma} f\left(I_{n}\right)\right\} .
\end{aligned}
$$

According to ( 3.27) and Lemma 3.4, the maximum of $f\left(I_{n}\right)$, subject to $I_{n} \geq 0$ and (3.38) is given by $\frac{1}{\gamma} \Omega_{n}$, where $\Omega_{n}$ is as in (3.27). Then, by Lemma 3.4 results in $I_{n}=\pi_{n}$. Therefore,

$$
V_{\tau_{n}}=(1-\varepsilon)\left[X_{\tau_{n}}-Y_{n}-\left(W_{\tau_{n}}-b\right)\right] \pi_{n},
$$

is optimal and unique for every $W_{\tau_{n}}$ which satisfies ( 3.31) and ( 3.37) when ( 3.38 ) holds. It can be shown that it is also optimal when (3.36) holds.

Since the second term of $\bar{S}\left(X_{\tau_{n}}\right)$ is always nonnegative, then $\bar{S}\left(X_{\tau_{n}}\right) \geq Q_{n}^{\nu} \frac{1}{\gamma}\left[W_{\tau_{n}}-b\right]^{\gamma}$. Therefore, ( 3.39) reduces to

$$
S\left(X_{\tau_{n}}\right)=\max _{\left\{W_{\tau_{n}}\right\}} S^{W_{\tau_{n}}}\left(X_{\tau_{n}}\right)
$$

where

$$
S^{W_{\tau_{n}}}\left(X_{\tau_{n}}\right)=Q_{n}^{\nu} \frac{1}{\gamma}\left(W_{\tau_{n}}-b\right)^{\gamma}+Q_{n+1}^{\nu} A_{n+1}^{-\nu} R_{n} \frac{1}{\gamma}\left[X_{\tau_{n}}-Y_{n}-\left(W_{\tau_{n}}-b\right)\right]^{\gamma}
$$


with $R_{n}=(1-\varepsilon)^{\gamma} e^{-\delta T_{n}} \Omega_{n}$. Since $u(C)=\frac{1}{\gamma} C^{\gamma}$ is strictly concave and $u^{\prime}(0)=\infty$, then $S^{W_{\tau_{n}}}$ is strictly concave and differentiable with respect to $W_{\tau_{n}}$, with a unique solution $W_{\tau_{n}}$ whenever $X_{\tau_{n}}-Y_{n} \geq 0$.

Differentiation of $S^{W_{\tau_{n}}}$ with respect to $W_{\tau_{n}}$ results in

$$
\frac{d S^{W_{\tau_{n}}}}{d W_{\tau_{n}}}=Q_{n}^{\nu}\left(W_{\tau_{n}}-b\right)^{-\nu}-A_{n+1}^{-\nu} Q_{n+1}^{\nu} R_{n}\left[X_{\tau_{n}}-Y_{n}-\left(W_{\tau_{n}}-b\right)\right]^{-\nu} .
$$

By setting $d S^{W_{\tau_{n}}} / d W_{\tau_{n}}=0$, then we have

$$
\left(W_{\tau_{n}}-b\right)^{-\nu}=A_{n+1}^{-\nu} Q_{n}^{-\nu} Q_{n+1}^{\nu} R_{n}\left[X_{\tau_{n}}-Y_{n}-\left(W_{\tau_{n}}-b\right)\right]^{-\nu}
$$

from which results in

$$
\left(W_{\tau_{n}}-b\right)\left[1+A_{n+1} Q_{n} Q_{n+1}^{-1} R_{n}^{-1 / \nu}\right]=A_{n+1} Q_{n} Q_{n+1}^{-1} R_{n}^{-1 / \nu}\left(X_{\tau_{n}}-Y_{n}\right) .
$$

Let define $F_{n}$ as the following :

$$
F_{n}=\frac{A_{n+1} Q_{n}}{A_{n+1} Q_{n}+Q_{n+1} R_{n}^{1 / \nu}} .
$$

Then the optimal withdrawal processes $W_{\tau_{n}}$ may be written as

$$
W_{\tau_{n}}=F_{n}\left(X_{\tau_{n}}-Y_{n}\right)+b
$$

By insertion of ( 3.43) into ( 3.41) results in the optimal investment strategy processes $V_{\tau_{n}}$ is in the form

$$
V_{\tau_{n}}=(1-\varepsilon)\left(1-F_{n}\right)\left(X_{\tau_{n}}-Y_{n}\right) \pi_{n}
$$

By substitution of ( 3.43 ) into ( 3.42 ) yields

$$
\begin{aligned}
S\left(X_{\tau_{n}}\right) & =Q_{n}^{\nu} F_{n}^{\gamma} \frac{1}{\gamma}\left(X_{\tau_{n}}-Y_{n}\right)^{\gamma}+Q_{n+1}^{\nu} A_{n+1}^{-\nu} R_{n} \\
& \times \frac{1}{\gamma}\left(X_{\tau_{n}}-Y_{n}\right)^{\gamma}\left(1-F_{n}\right)^{\gamma} \\
& =Q_{n}^{\nu} A_{n}^{-\nu} \frac{1}{\gamma}\left(X_{\tau_{n}}-Y_{n}\right)^{\gamma}\left[A_{n}^{\nu} F_{n}^{\gamma}\right. \\
& \left.+A_{n}^{\nu} A_{n+1}^{-\nu} Q_{n}^{-\nu} Q_{n+1}^{\nu} R_{n}\left(1-F_{n}\right)^{\gamma}\right] .
\end{aligned}
$$


On the other hand, from relation ( 3.22$), U\left(X_{\tau_{n}}\right)$ is given by

$$
U\left(X_{\tau_{n}}\right)=Q_{n}^{\nu} A_{n}^{-\nu} \frac{1}{\gamma}\left(X_{\tau_{n}}-Y_{n}\right)^{\gamma}
$$

This implies that $S\left(X_{\tau_{n}}\right)=U\left(X_{\tau_{n}}\right)$ if and only if

$$
A_{n}^{\nu} F_{n}^{\gamma}+A_{n}^{\nu} A_{n+1}^{-\nu} Q_{n}^{-\nu} Q_{n+1}^{\nu} R_{n}\left(1-F_{n}\right)^{\gamma}=1 .
$$

But relation $(*)$ holds if and only if

$$
\begin{aligned}
{\left[A_{n+1} Q_{n}+Q_{n+1} R_{n}^{1 / \nu}\right]^{\gamma} } & =A_{n}^{\nu} A_{n+1}^{-\nu} Q_{n}^{-\nu}+A_{n}^{\nu} A_{n+1}^{-\nu} Q_{n}^{-\nu} Q_{n+1} R_{n}^{1 / \nu} \\
& =A_{n}^{\nu} A_{n+1}^{-\nu} Q_{n}^{-\nu}\left[A_{n+1} Q_{n}+Q_{n+1} R_{n}^{1 / \nu}\right] .
\end{aligned}
$$

Therefore, $(*)$ holds if

$$
A_{n}=\frac{A_{n+1} Q_{n}}{A_{n+1} Q_{n}+Q_{n+1} R_{n}^{1 / \nu}}=F_{n} .
$$

Hence, the proof of the Theorem has been completed

Remark 3.3 For an equal intervals problem ${ }^{1}$, that is $T_{n}=T_{n+1} \quad \forall n$, we have that $A_{n}=A_{n+1}$ and that $Q_{n}=Q_{n+1}$. Applying these in relationship (3.25) results in

$$
A_{n}=\frac{A_{n} Q_{n}}{A_{n} Q_{n}+Q_{n} R_{n}^{1 / \nu}}=\frac{A_{n}}{A_{n}+R_{n}^{1 / \nu}}
$$

This implies that $A_{n}=1-R_{n}^{1 / \nu}$.

Corollary 3.2 Let the control problem be defined by problem (3.16)- (3.17). Furthermore, let $T_{n}$ be fixed for $n=1,2,3, \ldots$. Then $A_{n}$ as given by (3.25) has a property such that either $A_{n} \geq Q_{n}\left(1-R_{n}^{1 / \nu}\right)$, or $A_{n}<Q_{n}\left(1-R_{n}^{1 / \nu}\right)$.

\footnotetext{
${ }^{1}$ See Section 3.6 this thesis, or in Duffie and Sun [7]
} 
Proof : By Remark 3.3, for a given set of $\left\{T_{n}\right\}$ then $\left\{A_{n}\right\}$ will satisfy either $A_{n} \geq 1-R_{n}^{1 / \nu}$ or, $A_{n}<1-R_{n}^{1 / \nu}$. By keeping in mind that $0 \leq Q_{n} \leq 1$, then $\left\{A_{n}\right\}$ will also satisfy either $A_{n} \geq Q_{n}\left(1-R_{n}^{1 / \nu}\right)$ or $A_{n}<Q_{n}\left(1-R_{n}^{1 / \nu}\right)$.

Remark 3.4 Suppose that $A_{n+1}$ satisfies recurrence relationship (3.25). If $A_{n}$ as given by relation (3.25) has a property such that $A_{n} \geq Q_{n}\left(1-R_{n}^{1 / \nu}\right)$, then $A_{n+1}$ is such that

$$
\frac{A_{n+1}}{Q_{n+1}} \geq\left(1-R_{n}^{1 / \nu}\right) R_{n}^{1 / \nu}
$$

Proof : By applying $A_{n} \geq Q_{n}\left(1-R_{n}^{1 / \nu}\right)$, in relation (3.25) and arranging the terms, then $A_{n+1}$ satisfies

$$
\begin{aligned}
\frac{A_{n+1}}{Q_{n+1}} & =\frac{A_{n} R_{n}^{1 / \nu}}{Q_{n}\left(1-A_{n}\right)} \\
& \geq \frac{Q_{n}\left(1-R_{n}^{1 / \nu}\right) R_{n}^{1 / \nu}}{Q_{n}\left(1-A_{n}\right)} \\
& \geq \frac{\left(1-R_{n}^{1 / \nu}\right) R_{n}^{1 / \nu}}{\left[1-Q_{n}\left(1-R_{n}^{1 / \nu}\right)\right]}
\end{aligned}
$$

By its definition, $Q_{n}=1-e^{-\delta / \nu T_{n}} \in[0,1]$. Meanwhile, according to Lemma 3.6, $R_{n} \leq(1-\varepsilon)^{\gamma}$. Since $\varepsilon<1$ and $0<\gamma<1$, then $R_{n} \in[0,1]$. Therefore, $1-Q_{n}\left(1-R_{n}^{1 / \nu}\right) \leq 1$. Hence,

$$
\frac{A_{n+1}}{Q_{n+1}} \geq\left(1-R_{n}^{1 / \nu}\right) R_{n}^{1 / \nu}
$$


Remark 3.5 If at any $\tau_{n}, \quad W_{\tau_{n}}=b$, then the implications are as follows :

1. $Y_{n}=X_{\tau_{n}}$, by (3.23).

2. $T_{n}$ can be computed from (3.26) as

$$
0<T_{n}=\frac{1}{r} \ln \left[\frac{X_{\tau_{n+1}}}{\left(X_{\tau_{n}}-b\right)(1-\varepsilon)}\right]<\infty .
$$

3. $U\left(X_{\tau_{n}}\right)=0$, implying by (3.2) that $C_{t}=0$, almost every where for $t \geq \tau_{n}$. This can occur only for utility function which satisfy $u(0)=0$.

4. Case 2 and 3 imply that $W_{\tau_{n}}>b$ always in the case of infinite-time horizon

Corollary 3.3 It is possible that $W_{\tau_{n}}=b$, in which case

$$
T_{n}=\frac{1}{r} \ln \left[\frac{X_{\tau_{n+1}}}{\left(X_{\tau_{n}}-b\right)(1-\varepsilon)}\right]
$$

This implies that $b<W_{\tau_{n}}$ if the specified $T_{n}$ are not given by (3.45). 


\subsection{Optimal Transaction Intervals}

The aim of Section 3.5 is to establish an optimal transaction intervals $T_{n}$ for a given $(W, V, C)$. This is done by first deriving an equation satisfied by the interval $T_{n}$, and later by showing that a solution to that equation is the optimal choice for $T_{n}$.

Consider $U$ as in equation (3.16) which is given by

$$
U\left(X_{\tau_{n}}\right)=\max _{\left\{T_{n}, W_{\tau_{n}}, V_{\tau_{n}}\right\}}\left\{Q_{n}^{\nu} \frac{1}{\gamma}\left[W_{\tau_{n}}-b\right]^{\gamma}+e^{-\delta T_{n}} E\left[U\left(X_{\tau_{n+1}}\right) \mid \mathcal{H}_{\tau_{n}}\right]\right\} .
$$

By differentiation of function $U$ with respect to $W_{\tau_{n}}, V_{\tau_{n}}$ and $T_{n}$ respectively, and setting each of them equals to zero, then necessary conditions for ( 3.16 ) for all $n=1,2,3, \ldots$ are :

$$
\begin{gathered}
Q_{n}^{\nu}\left[W_{\tau_{n}}-b\right]^{-\nu}=(1-\varepsilon) e^{-(\delta-r) T_{n}} E\left[U^{\prime}\left(X_{\tau_{n+1}}\right) \mid \mathcal{H}_{\tau_{n}}\right], \\
E\left[\left(\Gamma_{n+1}-e^{r T_{n}}\right) U^{\prime}\left(X_{\tau_{n+1}}\right) \mid \mathcal{H}_{\tau_{n}}\right]=0, \\
\delta e^{-\frac{\delta}{\nu} T_{n}} Q_{n}^{-\gamma} \frac{1}{\gamma}\left[W_{\tau_{n}}-b\right]^{\gamma}= \\
e^{-\delta T_{n}}\left\{\delta E\left[U\left(X_{\tau_{n+1}}\right) \mid \mathcal{H}_{\tau_{n}}\right]-\frac{\partial E\left[U\left(X_{\tau_{n+1}}\right) \mid \mathcal{H}_{\tau_{n}}\right]}{\partial T_{n}}\right\} .
\end{gathered}
$$

Application of Itós formula ${ }^{2}$ on the function $U$, results in

$$
U\left(X_{\tau_{n+1}}\right)-U\left(X_{\tau_{n}}\right)=\int_{\tau_{n}+}^{\tau_{n+1}}\left[U^{\prime}\left(X_{t}\right) d X_{t}+\frac{1}{2} U^{\prime \prime}\left(X_{t}\right) d X_{t}^{2}\right] .
$$

From the definition of the wealth process $X_{\tau_{n+1}}$ in (3.17), then for any $t \in\left[\tau_{n}, \tau_{n+1}\right)$, we have

$$
X_{t}=(1-\varepsilon)\left[X_{\tau_{n}}-W_{\tau_{n}}\right] e^{r\left(t-\tau_{n}\right)}+V_{\tau_{n}}\left(\Gamma_{t}-e^{r\left(t-\tau_{n}\right)}\right) .
$$

\footnotetext{
${ }^{2}$ See for example Theorem 32 of Protter [22]
} 
Therefore, the following holds for any $t \in\left[\tau_{n}, \tau_{n+1}\right)$,

$$
d X_{t}=\left[r\left(X_{t}-G_{t}\right)+\alpha G_{t}\right] d t+\sigma G_{t} d B_{t}
$$

and $d X_{t}^{2}=\sigma^{2} G_{t}^{2} d t$, with $G_{t} \equiv V_{\tau_{n}} \Gamma_{t}$, and where $\Gamma_{t}$ is given by

$$
\Gamma_{t}=\exp \left[\left(\alpha-\frac{1}{2} \sigma^{2}\right)\left(t-\tau_{n}\right)+\sigma\left(B_{t}-B_{\tau_{n}}\right)\right] .
$$

By using the above results in (3.47), then

$$
\begin{aligned}
U\left(X_{\tau_{n+1}}\right)-U\left(X_{\tau_{n}}\right) & =\int_{\tau_{n}+}^{\tau_{n+1}}\left\{\left[r\left(X_{t}-G_{t}\right)+\alpha G_{t}\right] U^{\prime}\left(X_{t}\right) d t\right. \\
& \left.+\frac{1}{2} \sigma^{2} G_{t}^{2} U^{\prime \prime}\left(X_{t}\right) d t+\sigma G_{t} U^{\prime}\left(X_{t}\right) d B_{t}\right\} .
\end{aligned}
$$

Let processes $\left\{Z_{t}\right\}$ be defined as the following :

$$
Z_{t}=\int_{\tau_{n}}^{t} \sigma G_{s} U\left(X_{s}\right) d B_{s}, \quad t \in\left(\tau_{n}, \tau_{n+1}\right]
$$

Apparently processes $\left\{Z_{t}\right\}$ is a martingale. ${ }^{3}$ Therefore,

$$
\begin{aligned}
\frac{\partial E\left[U\left(X_{\tau_{n+1}}\right) \mid \mathcal{H}_{\tau_{n}}\right]}{\partial T_{n}} & =E\left[\left[r\left(X_{\tau_{n+1}}-G_{\tau_{n+1}}\right)+\alpha G_{\tau_{n+1}}\right] U^{\prime}\left(X_{\tau_{n+1}}\right)\right. \\
& \left.+\frac{1}{2} \sigma^{2} G_{\tau_{n+1}}^{2} U^{\prime \prime}\left(X_{\tau_{n+1}}\right) \mid \mathcal{H}_{\tau_{n}}\right]
\end{aligned}
$$

Substitution of relation (3.48) into (3.46) results in

$$
\begin{aligned}
& \delta e^{-\frac{\delta}{\nu} T_{n}} Q_{n}^{-\gamma} \frac{1}{\gamma}\left[W_{\tau_{n}}-b\right]^{\gamma}= \\
& \delta e^{-\delta T_{n}} E\left[U\left(X_{\tau_{n+1}}\right) \mid \mathcal{H}_{\tau_{n}}\right]-e^{-\frac{\delta}{\nu} T_{n}} E\left[\left[r\left(X_{\tau_{n+1}}-G_{\tau_{n}+1}\right)\right.\right. \\
& \left.\left.\quad+\alpha G_{\tau_{n+1}}\right] U^{\prime}\left(X_{\tau_{n+1}}\right)+\frac{1}{2} \sigma^{2} G_{\tau_{n+1}}^{2} U^{\prime \prime}\left(X_{\tau_{n+1}}\right) \mid \mathcal{H}_{\tau_{n}}\right] .
\end{aligned}
$$

\footnotetext{
${ }^{3}$ For details, see appendix 3 of $[7]$
} 
Remark 3.6 Duffie and Sun in [7] have used $V_{\tau_{n+1}}$ in place of $G_{\tau_{n+1}}$.

Notice that by insertion of (3.23) and ( 3.24$)$ into (3.17), then the total wealth process $X_{\tau_{n+1}}$ may be re-written as

$$
X_{\tau_{n+1}}=(1-\varepsilon)\left(1-A_{n}\right)\left(X_{\tau_{n}}-Y_{n}\right)\left[e^{r T_{n}}+\pi_{n}\left(\Gamma_{n+1}-e^{r T_{n}}\right)\right]+Y_{n+1}
$$

Since $G_{\tau_{n+1}}=V_{\tau_{n}} \Gamma_{n+1}$, then by application of ( 3.24$)$ we also have

$$
G_{\tau_{n+1}}=(1-\varepsilon)\left(1-A_{n}\right)\left(X_{\tau_{n}}-Y_{n}\right) \pi_{n} \Gamma_{n+1}
$$

Applying ( 3.50) in (3.22) then we have the following equations :

$$
\begin{aligned}
E\left[U\left(X_{\tau_{n+1}}\right) \mid \mathcal{H}_{\tau_{n}}\right] & =(1-\varepsilon)^{\gamma}\left(1-A_{n}\right)^{\gamma} \frac{1}{\gamma}\left(X_{\tau_{n}}-Y_{n}\right)^{\gamma} \\
& \times E\left(\left[e^{r T_{n}}+\pi_{n}\left(\Gamma_{n+1}-e^{r T_{n}}\right)\right]^{\gamma}\right) Q_{n+1}^{\nu} A_{n+1}^{-\nu}, \\
E\left[U^{\prime}\left(X_{\tau_{n+1}}\right) \mid \mathcal{H}_{\tau_{n}}\right] & =(1-\varepsilon)^{-\nu}\left(1-A_{n}\right)^{-\nu}\left(X_{\tau_{n}}-Y_{n}\right)^{-\nu} \\
& \times E\left(\left[e^{r T_{n}}+\pi_{n}\left(\Gamma_{n+1}-e^{r T_{n}}\right)\right]^{-\nu}\right) Q_{n+1}^{\nu} A_{n+1}^{-\nu}, \\
E\left[U^{\prime \prime}\left(X_{\tau_{n+1}}\right) \mid \mathcal{H}_{\tau_{n}}\right] & =(1-\varepsilon)^{-\nu-1}\left(1-A_{n}\right)^{-\nu-1}\left(X_{\tau_{n}}-Y_{n}\right)^{-\nu-1} \\
& \times(-\nu) E\left(\left[e^{r T_{n}}+\pi_{n}\left(\Gamma_{n+1}-e^{r T_{n}}\right)\right]^{-\nu-1}\right) Q_{n+1}^{\nu} A_{n+1}^{-\nu} .
\end{aligned}
$$

From the definition of $\Omega_{n}$ in (3.27), then its derivative with respect to $\pi_{n}$ gives

$$
E\left(\left[e^{r T_{n}}+\pi_{n}\left(\Gamma_{n+1}-e^{r T_{n}}\right)\right]^{-\nu}\left[\Gamma_{n+1}-e^{r T_{n}}\right]\right)=0 .
$$

This implies that the following relations hold :

$$
\begin{aligned}
\Omega_{n} & =E\left(e^{r T_{n}}\left[e^{r T_{n}}+\pi_{n}\left(\Gamma_{n+1}-e^{r T_{n}}\right)\right]^{-\nu}\right), \\
& =E\left(\Gamma_{n+1}\left[e^{r T_{n}}+\pi_{n}\left(\Gamma_{n+1}-e^{r T_{n}}\right)\right]^{-\nu}\right), \\
& =E\left(\Gamma_{n+1}^{2}\left[e^{r T_{n}}+\pi_{n}\left(\Gamma_{n+1}-e^{r T_{n}}\right)\right]^{-\nu-1}\right) .
\end{aligned}
$$


By substituting previous equations and (3.23), ( 3.51), ( 3.55), ( 3.56), ( 3.57$)$ into ( 3.49$)$, and by dividing all terms by $(1 / \gamma)\left(X_{\tau_{n}}-Y_{n}\right)^{\gamma-1}$, and by rearranging the terms, then we have the following equation :

$$
\begin{gathered}
\left(X_{\tau_{n}}-Y_{n}\right)\left\{\delta e^{-\frac{\delta}{\nu} T_{n}} Q_{n}^{-\gamma} A_{n}^{\gamma}+Q_{n+1}^{\nu} A_{n+1}^{-\nu}\left(1-A_{n}\right)^{\gamma} R_{n}[-\delta+\gamma r\right. \\
\left.\left.+\gamma(\alpha-r) \pi_{n}-\frac{1}{2} \sigma^{2} \gamma(1-\gamma) \pi_{n}^{2}\right]\right\} \\
+\gamma r(1-\varepsilon)^{-1} e^{-r T_{n}} Q_{n+1}^{\nu} A_{n+1}^{-\nu}\left(1-A_{n}\right)^{-\nu} R_{n} Y_{n+1}=0 .
\end{gathered}
$$

By applications of $A_{n}$ of (3.25) and $Y_{n}$ of (3.26) in (3.58), then we have

$$
\begin{aligned}
& \left(X_{\tau_{n}}-Y_{n}\right)\left\{\delta e^{-\frac{\delta}{\nu} T_{n}} \frac{A_{n+1}^{\gamma}}{\left[A_{n+1} Q_{n}+Q_{n+1} R_{n}^{1 / \nu}\right] \gamma}+Q_{n+1}^{\nu} A_{n+1}^{-\nu} R_{n}\right. \\
& \left.\quad \times \frac{Q_{n+1}^{\gamma} R_{n}^{\gamma / \nu}}{\left[A_{n+1} Q_{n}+Q_{n+1} R_{n}^{1 / \nu}\right] \gamma}\left[-\delta+\gamma r+\gamma(\alpha-r) \pi_{n}-\frac{1}{2} \sigma^{2} \gamma(1-\gamma) \pi_{n}^{2}\right]\right\} \\
& \quad+\frac{\gamma r Y_{n+1} R_{n}}{(1-\varepsilon) e^{r T_{n}}} Q_{n+1}^{\nu} A_{n+1}^{-\nu} \frac{Q_{n+1}^{-\nu} R_{n}^{-\nu / \nu}}{\left[A_{n+1} Q_{n}+Q_{n+1} R_{n}\right]^{-\nu}}=0
\end{aligned}
$$

Finally, by dividing all terms of $(3.59)$ by $\frac{A_{n+1}^{-\nu}}{\left[A_{n+1} Q_{n}+Q_{n+1} R_{n}^{1 / \nu}\right]^{\gamma}}$ results in the following relation :

$$
g\left(T_{n}\right)+\left(X_{\tau_{n}}-Y_{n}\right) h\left(T_{n}\right)=0,
$$

where $g$ and $h$ are defined as the following :

$$
\begin{aligned}
g\left(T_{n}\right)= & \gamma r(1-\varepsilon)^{-1} e^{-r T_{n}} Y_{n+1}\left[A_{n+1} Q_{n}+Q_{n+1} R_{n}^{1 / \nu}\right] \\
h\left(T_{n}\right) & =\delta e^{-\frac{\delta}{\nu} T_{n}} A_{n+1}+Q_{n+1} R_{n}^{1 / \nu}\left[-\delta+\gamma r+\gamma(\alpha-r) \pi_{n}\right. \\
& \left.-\frac{1}{2} \sigma^{2} \gamma(1-\gamma) \pi_{n}^{2}\right] .
\end{aligned}
$$

Therefore the proof of the following theorem has been completed. 
Theorem 3.2 Let the problem faced by an investor satisfy relation ( 3.16) subject to (3.17). Then the optimal transaction intervals $T_{n}, n=1,2,3, \ldots$ satisfy

$$
g\left(T_{n}\right)+\left(X_{\tau_{n}}-Y_{n}\right) h\left(T_{n}\right)=0,
$$

where $g$ and $h$ are defined by

$$
\begin{aligned}
g\left(T_{n}\right)= & \gamma r(1-\varepsilon)^{-1} e^{-r T_{n}} Y_{n+1}\left[A_{n+1} Q_{n}+Q_{n+1} R_{n}^{1 / \nu}\right], \\
h\left(T_{n}\right) & =\delta e^{-\frac{\delta}{\nu} T_{n}} A_{n+1}+Q_{n+1} R_{n}^{1 / \nu}\left[-\delta+\gamma r+\gamma(\alpha-r) \pi_{n}\right. \\
& \left.-\frac{1}{2} \sigma^{2} \gamma(1-\gamma) \pi_{n}^{2}\right] .
\end{aligned}
$$

Theorem 3.3 Suppose that $\alpha, \delta, \varepsilon, \gamma, r, \sigma$ satisfying the following conditions :

$$
\begin{aligned}
& \text { 1. } \alpha-r \geq \frac{1}{2} \sigma^{2}(1-\gamma) \\
& \text { 2. } \max (\gamma \alpha, \gamma r)<\delta<\min \left(\frac{\gamma r}{(1-\varepsilon)^{\gamma / \nu}}, r-\gamma r\right)
\end{aligned}
$$

If $A_{n}$ as given by (3.25) has a property such that $A_{n} \geq Q_{n}\left(1-R_{n}^{1 / \nu}\right)$, then the equation

$$
g\left(T_{n}\right)+\left(X_{\tau_{n}}-Y_{n}\right) h\left(T_{n}\right)=0
$$

as defined in Theorem 3.2 has a solution.

Proof : Let $H\left(T_{n}\right)=g\left(T_{n}\right)+\left(X_{\tau_{n}}-Y_{n}\right) h\left(T_{n}\right)$, where $g$ and $h$ are defined by (3.64) and (3.65) respectively. Note that $H$ is a continuous function of $T_{n}$. It will be shown that $H>0$ as $T_{n} \rightarrow 0^{+}$and $H<0$ as $T_{n} \rightarrow+\infty$. 
From the definition of functions $g$ and $h$, then we have

$$
\begin{aligned}
\lim _{T_{n} \rightarrow 0^{+}} H\left(T_{n}\right) & =\lim _{T_{n} \rightarrow 0^{+}} \gamma r(1-\varepsilon)^{-1} e^{-r T_{n}} Y_{n+1}\left[A_{n+1} Q_{n}+Q_{n+1} R_{n}^{1 / \nu}\right] \\
& +\lim _{T_{n} \rightarrow 0^{+}} Q_{n+1}\left(X_{\tau_{n}}-Y_{n}\right)\left\{\delta e^{-\delta / \nu T_{n}} \frac{A_{n+1}}{Q_{n+1}}\right. \\
& \left.+R_{n}^{1 / \nu}\left[-\delta+\gamma r+\gamma(\alpha-r) \pi_{n}-1 / 2 \sigma^{2} \gamma(1-\gamma) \pi_{n}^{2}\right]\right\} .
\end{aligned}
$$

By Corollary 3.2, either $A_{n} \geq Q_{n}\left(1-R_{n}^{1 / \nu}\right)$, or $A_{n}<Q_{n}\left(1-R_{n}^{1 / \nu}\right)$.

But we have assumed that $A_{n} \geq Q_{n}\left(1-R_{n}^{1 / \nu}\right)$. Therefore, by Remark 3.4, $\frac{A_{n+1}}{Q_{n+1}} \geq\left(1-R_{n}^{1 / \nu}\right) R_{n}^{1 / \nu}$. Furthermore, $Y_{n}=b+(1-\varepsilon)^{-1} e^{-r T_{n}} Y_{n+1}$ by relation ( 3.26). Therefore,

$$
\begin{aligned}
\lim _{T_{n} \rightarrow 0^{+}} H\left(T_{n}\right) & \geq \lim _{T_{n} \rightarrow 0^{+}} \gamma r(1-\varepsilon)^{-1} e^{-r T_{n}} Y_{n+1}\left[A_{n+1} Q_{n}+Q_{n+1} R_{n}^{1 / \nu}\right] \\
& +\lim _{T_{n} \rightarrow 0^{+}} Q_{n+1}\left(X_{\tau_{n}}-b-(1-\varepsilon)^{-1} e^{-r T_{n}} Y_{n+1}\right) \\
& \times\left\{\delta e^{-\delta / \nu T_{n}}\left(1-R_{n}^{1 / \nu}\right) R_{n}^{1 / \nu}+R_{n}^{1 / \nu}[-\delta+\gamma r\right. \\
& \left.\left.+\gamma(\alpha-r) \pi_{n}-1 / 2 \sigma^{2} \gamma(1-\gamma) \pi_{n}^{2}\right]\right\} .
\end{aligned}
$$

By the assumption of the theorem, $(\alpha-r) \geq \frac{1}{2} \sigma^{2}(1-\gamma)$. In addition, by its definition, $0 \leq \pi_{n} \leq 1$. These imply that

$$
-\delta+\gamma r+\gamma(\alpha-r) \pi_{n}-\frac{1}{2} \sigma^{2} \gamma(1-\gamma) \pi_{n}^{2} \geq-\delta+\gamma r .
$$

And since $\lim _{T_{n} \rightarrow 0^{+}} R_{n}^{1 / \nu}=(1-\varepsilon)^{\gamma / \nu}$, then we have that

$$
\begin{aligned}
\lim _{T_{n} \rightarrow 0^{+}} H\left(T_{n}\right) & \geq \gamma r(1-\varepsilon)^{-1} Y_{n+1} Q_{n+1}(1-\varepsilon)^{\gamma / \nu} \\
& +Q_{n+1}\left(X_{\tau_{n}}-b-(1-\varepsilon)^{-1} Y_{n+1}\right) \\
& \times\left\{\delta\left[1-(1-\varepsilon)^{\gamma / \nu}\right](1-\varepsilon)^{\gamma / \nu}+(1-\varepsilon)^{\gamma / \nu}[-\delta+\gamma r]\right\} .
\end{aligned}
$$

By the assumption of the Theorem, $\delta<\frac{\gamma r}{(1-\varepsilon)^{\gamma / \nu}}$. This implies that

$$
\delta\left[1-(1-\varepsilon)^{\gamma / \nu}\right](1-\varepsilon)^{\gamma / \nu}+(1-\varepsilon)^{\gamma / \nu}[-\delta+\gamma r]>0 .
$$


Therefore,

$$
\lim _{T_{n} \rightarrow 0^{+}} H\left(T_{n}\right)>\gamma r(1-\varepsilon)^{-1+\gamma / \nu} Q_{n+1} Y_{n+1} .
$$

Since $\gamma, r, \varepsilon, Y_{n+1}, Q_{n+1}$ are nonnegatives, hence

$$
\lim _{T_{n} \rightarrow 0^{+}} H\left(T_{n}\right)>0 .
$$

On the other hand, since $A_{n+1}, Q_{n+1}, Q_{n}$ are nonnegatives and less than one, and $R_{n} \leq(1-\varepsilon)^{\gamma}$, then

$$
\begin{aligned}
H\left(T_{n}\right) & =g\left(T_{n}\right)+\left(X_{\tau_{n}}-Y_{n}\right) h\left(T_{n}\right) \\
& =\frac{\gamma r}{(1-\varepsilon) e^{r T_{n}}} Y_{n+1}\left[A_{n+1} Q_{n}+Q_{n+1} R_{n}^{1 / \nu}\right] \\
& +\left(X_{\tau_{n}}-Y_{n}\right)\left\{\delta e^{-\frac{\delta}{\nu} T_{n}} A_{n+1}+Q_{n+1} R_{n}^{1 / \nu}\right. \\
& \left.\times\left[-\delta+\gamma r+\gamma(\alpha-r) \pi_{n}-\frac{1}{2} \sigma^{2} \gamma(1-\gamma) \pi_{n}^{2}\right]\right\} \\
& \leq \frac{\gamma r Y_{n+1}\left[1+(1-\varepsilon)^{\gamma / \nu}\right]}{(1-\varepsilon) e^{r T_{n}}} \\
& +\left(X_{\tau_{n}}-Y_{n}\right)\left\{\delta e^{-\frac{\delta}{\nu} T_{n}}+Q_{n+1} R_{n}^{1 / \nu}[-\delta+\max (\gamma \alpha, \gamma r)]\right\} .
\end{aligned}
$$

By re-arranging all terms, then

$$
\begin{aligned}
H\left(T_{n}\right) & \leq e^{-\frac{\delta}{\nu} T_{n}}\left(X_{\tau_{n}}-Y_{n}\right)\left\{\frac{\gamma r Y_{n+1} e^{\frac{\delta}{\nu} T_{n}}\left[1+(1-\varepsilon)^{\gamma / \nu}\right]}{\left(X_{\tau_{n}}-Y_{n}\right)(1-\varepsilon) e^{r T_{n}}}\right. \\
& \left.+\delta+e^{\delta / \nu T_{n}} Q_{n+1} R_{n}^{1 / \nu}[-\delta+\max (\gamma \alpha, \gamma r)]\right\} .
\end{aligned}
$$

Since, by assumptions of Theorem 3.3 that $\delta>\max (\gamma \alpha, \gamma r)$, and $r>\delta / \nu$, then $H\left(T_{n}\right)<0$ for large $T_{n}$. It has been shown that $H\left(T_{n}\right)>0$ as $T_{n} \rightarrow 0^{+}$, and $H\left(T_{n}\right)<0$ for large $T_{n}$. Since $H\left(T_{n}\right)$ is a continuous function in $T_{n}$, then there exists $\hat{T}_{n}$, such that $H\left(\hat{T}_{n}\right)=0$. Therefore, the proof of Theorem 3.3 has been completed 
Theorem 3.4 Suppose that $\alpha, \delta, \varepsilon, \gamma, r, \sigma$ satisfying the following conditions :

$$
\begin{aligned}
& \text { 1. } \alpha-r \geq \frac{1}{2} \sigma^{2}(1-\gamma) \\
& \text { 2. } \max (\gamma \alpha, \gamma r)<\delta<\min \left(\frac{\gamma r}{(1-\varepsilon)^{\gamma / \nu}}, r-\gamma r\right)
\end{aligned}
$$

If $A_{n}$ as given by (3.25) has the property such that $A_{n} \geq Q_{n}\left(1-R_{n}^{1 / \nu}\right)$, then an optimal policy $(T, W, V, C)$ exists.

Proof : By Remark 3.4, $\frac{A_{n+1}}{Q_{n+1}} \geq\left(1-R_{n}^{1 / \nu}\right) R_{n}^{1 / \nu}$ for $A_{n} \geq Q_{n}\left(1-R_{n}^{1 / \nu}\right)$. By Theorem 3.3, then there exists a scalar $\hat{T}_{n}>0$ such that $H\left(\hat{T}_{n}\right)=0$. The arguments in the proof of Theorem 3.3 show that one of the solutions, say $\hat{T}_{n}$, corresponds to the maximum of (3.16).

Now consider the following withdrawal and investment policy and function $f$, for $n=1,2,3, \ldots$

$$
\begin{aligned}
f\left(X_{\tau_{n}}\right) & =Q_{n}^{\nu} A_{n}^{-\nu} \frac{1}{\gamma}\left(X_{\tau_{n}}-Y_{n}\right)^{\gamma}, \\
W_{\tau_{n}} & =A_{n}\left(X_{\tau_{n}}-Y_{n}\right)+b, \\
V_{\tau_{n}} & =(1-\varepsilon)\left(1-A_{n}\right)\left(X_{\tau_{n}}-Y_{n}\right) \pi_{n}, \\
T_{n} & =\hat{T}_{n} .
\end{aligned}
$$

From the preceeding calculations, we know that $f\left(X_{\tau_{n}}\right)$ satisfies ( 3.16) and that $\left(T_{n}, W_{\tau_{n}}, V_{\tau_{n}}\right)$ achieves the maximum in (3.18) $\forall n$. It will be shown that $\lim _{n \rightarrow \infty} E\left[e^{-\delta \tau_{n}} f\left(X_{\tau_{n}}\right)\right]=0$. By using (3.66), then

$$
E\left[e^{-\delta \tau_{n+1}} f\left(X_{\tau_{n+1}}\right) \mid \mathcal{H}_{\tau_{n}}\right]=Q_{n+1}^{\nu} A_{n+1}^{-\nu} e^{-\delta \tau_{n+1}} \frac{1}{\gamma} E\left[\left(X_{\tau_{n+1}}-Y_{n+1}\right)^{\gamma} \mid \mathcal{H}_{\tau_{n}}\right],
$$


with the total wealth $X_{\tau_{n+1}}$ is given by $(3.50)$.

By substitution of ( 3.67 ) and ( 3.68) into the total wealth $X_{\tau_{n+1}}$ of (3.50), then the expectation factor on the right hand side of ( 3.70) may be written as

$$
E\left[\left(X_{\tau_{n+1}}-Y_{n+1}\right)^{\gamma} \mid \mathcal{H}_{\tau_{n}}\right]=(1-\varepsilon)^{\gamma} \Omega_{n}\left(1-A_{n}\right)^{\gamma}\left[\left(X_{\tau_{n}}-Y_{n}\right)^{\gamma} \mid \mathcal{H}_{\tau_{n-1}}\right] .
$$

Since $R_{n}=(1-\varepsilon)^{\gamma} e^{-\delta T_{n}} \Omega_{n}$, and $\tau_{n+1}=T_{n}+\tau_{n}$, then the right hand side of ( 3.70$)$ may be written as

$$
\begin{aligned}
\frac{1}{\gamma} & Q_{n+1}^{\nu} A_{n+1}^{-\nu} e^{-\delta \tau_{n+1}} E\left[\left(X_{\tau_{n+1}}-Y_{n+1}\right)^{\gamma} \mid \mathcal{H}_{\tau_{n}}\right] \\
& =\frac{1}{\gamma} Q_{n+1}^{\nu} A_{n+1}^{-\nu} e^{-\delta T_{n}}(1-\varepsilon)^{\gamma} \Omega_{n}\left(1-A_{n}\right)^{\gamma} e^{-\delta \tau_{n}}\left[\left(X_{\tau_{n}}-Y_{n}\right)^{\gamma} \mid \mathcal{H}_{\tau_{n-1}}\right] \\
& =\frac{1}{\gamma} Q_{n+1}^{\nu} A_{n+1}^{-\nu} R_{n}\left(1-A_{n}\right)^{\gamma} e^{-\delta \tau_{n}}\left[\left(X_{\tau_{n}}-Y_{n}\right)^{\gamma} \mid \mathcal{H}_{\tau_{n-1}}\right] .
\end{aligned}
$$

Hence, we have

$$
E\left[e^{-\delta \tau_{n+1}} f\left(X_{\tau_{n+1}}\right) \mid \mathcal{H}_{\tau_{n}}\right]=\frac{1}{\gamma} Q_{n+1}^{\nu} A_{n+1}^{-\nu} R_{n}\left(1-A_{n}\right)^{\gamma} e^{-\delta \tau_{n}}\left[\left(X_{\tau_{n}}-Y_{n}\right)^{\gamma} \mid \mathcal{H}_{\tau_{n-1}}\right]
$$

Let $D=\frac{1}{\gamma} Q_{n+1}^{\nu} A_{n+1}^{-\nu}$.

Then by induction we have the following :

$$
\begin{aligned}
E & {\left[e^{-\delta \tau_{n+1}} f\left(X_{\tau_{n+1}}\right)\right] } \\
& =D R_{n}\left(1-A_{n}\right)^{\gamma} e^{-\delta \tau_{n}}\left[\left(X_{\tau_{n}}-Y_{n}\right)^{\gamma} \mid \mathcal{H}_{\tau_{n-1}}\right] \\
& =D R_{n}\left(1-A_{n}\right)^{\gamma} R_{n-1}\left(1-A_{n-1}\right)^{\gamma} e^{-\delta \tau_{n-1}}\left[\left(X_{\tau_{n-1}}-Y_{n-1}\right)^{\gamma} \mid \mathcal{H}_{\tau_{n-2}}\right] \\
& =D R_{n}\left(1-A_{n}\right)^{\gamma} \ldots R_{1}\left(1-F_{1}\right)^{\gamma} e^{-\delta \tau_{1}}\left[\left(X_{\tau_{1}}-Y_{1}\right)^{\gamma} \mid \mathcal{H}_{\tau_{1}}\right] \\
& \leq D X_{0}^{\gamma} \prod_{k=1}^{n}\left[R_{k}\left(1-A_{k}\right)^{\gamma}\right] \leq D X_{0}^{\gamma} \prod_{k=1}^{n}\left[(1-\varepsilon)^{\gamma / \nu}\right],
\end{aligned}
$$


as $0<1-A_{n}<1$, and $R_{n} \leq(1-\varepsilon)^{\gamma / \nu} \forall n$. Since $D$ is bounded, then $\lim _{n \rightarrow \infty} E\left[e^{-\delta \tau_{n}} f\left(X_{\tau_{n}}\right)\right]=0$.

Therefore, condition (ii) of Lemma 3.3 is satisfied. Hence, by Lemma 3.3, the proof of Theorem 3.4 has been completed 


\subsection{Equal Intervals}

Now consider the case of transaction costs $b=0$. Let transaction intervals $T_{1}=T_{2}=\ldots=K$. This implies that $\tau_{n}=(n-1) K$. With $T_{n}$ are equals for all $\mathrm{n}$, then $A_{n+1}=A_{n}, \quad Q_{n+1}=Q_{n}, \quad Y_{n+1}=Y_{n}$. By replacing $A_{n+1}$ with $A_{n}$ and $Q_{n+1}$ with $Q_{n}$ in (3.25), and replacing $Y_{n+1}$ with $Y_{n}$ in (3.26), respectively, result in

$$
A_{n}=1-\left[(1-\varepsilon)^{\gamma} e^{-\delta T_{n}} \Omega_{n}\right]^{1 / \nu}
$$

and

$$
Y_{n}=\frac{(1-\varepsilon) e^{r T_{n}} b}{(1-\varepsilon) e^{r T_{n}}-1}
$$

Note that transaction costs function $b=0$ implies $Y_{n}=0$. This implies that $g\left(T_{n}\right)+\left(X_{\tau_{n}}-Y_{n}\right) h\left(T_{n}\right)=0$ is equivalent to $h\left(T_{n}\right)=0$, where $h$ is given by

$$
\begin{aligned}
h\left(T_{n}\right) & =\delta e^{-\frac{\delta}{\nu} T_{n}} A_{n}+Q_{n} R_{n}^{1 / \nu}\left[-\delta+\gamma r+\gamma(\alpha-r) \pi_{n}\right. \\
& \left.-\frac{1}{2} \sigma^{2} \gamma \nu \pi_{n}^{2}\right] .
\end{aligned}
$$

The following result is proved in [7], however the proof is incomplete.

Theorem 3.5 Suppose that $\delta>\max (\gamma \alpha, \gamma r)$, and that $b=0$.

Then $h\left(T_{n}\right)=0$ has a solution.

Proof : From its definition, $h$ is a continuous function in $T_{n}$. It will be shown that $h>0$, as $T_{n}$ approaches zero, and $h<0$, as $T_{n}$ goes toward infinity. Since $Q_{n}=1-e^{-\frac{\delta}{\nu} T_{n}}$, then

$$
\lim _{T_{n} \rightarrow 0^{+}} h\left(T_{n}\right)=\lim _{T_{n} \rightarrow 0^{+}} \delta e^{-\frac{\delta}{\nu} T_{n}} A_{n} .
$$


Note that

$\lim _{T_{n} \rightarrow 0^{+}} \exp \left[(-\delta+\gamma r) T_{n}\right]=1, \quad$ and $\quad \lim _{T_{n} \rightarrow 0^{+}} \exp \left[(-\delta+\max (\gamma \alpha, \gamma r)) T_{n}\right]=1$.

Since

$$
e^{(-\delta+\gamma r) T_{n}} \leq e^{-\delta T_{n}} \Omega_{n} \leq e^{[-\delta+\max (\gamma \alpha, \gamma r)] T_{n}},
$$

then $\lim _{T_{n} \rightarrow 0^{+}} e^{-\delta T_{n}} \Omega_{n}=1$. Therefore,

$$
\lim _{T_{n} \rightarrow 0^{+}} h\left(T_{n}\right)=\delta\left[1-(1-\varepsilon)^{\gamma / \nu}\right]>0 .
$$

On the other hand,

$$
\begin{aligned}
h\left(T_{n}\right) & \leq \delta e^{-\frac{\delta}{\nu} T_{n}} A_{n}+\left(1-A_{n}\right) Q_{n} \\
& \times\left[\gamma r+\gamma(\alpha-r) \pi_{n}-\delta\right] \\
& \leq e^{-\frac{\delta}{\nu} T_{n}}\left[\delta+(\max (\gamma \alpha, \gamma r)-\delta)(1-\varepsilon)^{\gamma / \nu} e^{\frac{\gamma r}{\nu} T_{n}}\right] .
\end{aligned}
$$

Since by the assumption of Theorem 3.5 that $\delta>\max (\gamma \alpha, \gamma r)$, then for large $T_{n}, h\left(T_{n}\right)<0$. Since $h$ is continuous in $T_{n}$, therefore there exists $\bar{T}_{n}$ such that $h\left(\bar{T}_{n}\right)=0$. Hence, the proof of Theorem 3.5 has been completed

Theorem 3.6 Suppose that $\delta>\max (\gamma \alpha, \gamma r)$ and that $b=0$. Then an optimal policy $(T, W, V, C)$ exists and the optimal transaction intervals $\left\{T_{n}\right\}$ are equal.

Proof : The proof is as in [7]. With $b=0, H\left(T_{n}\right)=0$ is equivalent to $h\left(T_{n}\right)=0$, for all $\mathrm{n}$. And by Theorem 3.5, $h\left(T_{n}\right)=0$, has a solution. Therefore, there exists $\bar{T}_{n}$ such that $h\left(\bar{T}_{n}\right)=0$. 
Now consider the following withdrawal and investment policy and function $f$, for $\mathrm{n}=1,2,3, \ldots$

$$
\begin{aligned}
f\left(X_{\tau_{n}}\right) & =Q_{n}^{\nu} A_{n}^{-\nu} \frac{1}{\gamma} X_{\tau_{n}}^{\gamma}, \\
W_{\tau_{n}} & =A_{n} X_{\tau_{n}}, \\
V_{\tau_{n}} & =(1-\varepsilon)\left(1-A_{n}\right) X_{\tau_{n}} \pi_{n}, \\
T_{n} & =\bar{K} .
\end{aligned}
$$

From the preceeding calculations, we know that that $f\left(X_{\tau_{n}}\right)$ satisfies ( 3.16) and that $\left(T_{n}, W_{\tau_{n}}, V_{\tau_{n}}\right)$ achieves the maximum in (3.18) $\forall n$.

Let $D \equiv(1 / \gamma) Q_{n+1}^{\nu} A_{n+1}^{-\nu}$. Therefore,

$$
\begin{aligned}
E & {\left[e^{-\delta \tau_{n+1}} f\left(X_{\tau_{n+1}}\right) \mid \mathcal{H}_{\tau_{n}}\right]=e^{-\delta\left(T_{n}+\tau_{n}\right)} D E\left[X_{\tau_{n+1}}^{\gamma} \mid \mathcal{H}_{\tau_{n}}\right] } \\
& =D e^{-\delta T_{n}}(1-\varepsilon)^{\gamma} \Omega_{n}\left(1-A_{n}\right)^{\gamma} e^{-\delta \tau_{n}}\left[X_{\tau_{n}}^{\gamma} \mid \mathcal{H}_{\tau_{n-1}}\right] \\
& =D R_{n}\left(1-A_{n}\right)^{\gamma} e^{-\delta \tau_{n}}\left[X_{\tau_{n}}^{\gamma} \mid \mathcal{H}_{\tau_{n-1}}\right] \\
& =D R_{n}\left(1-A_{n}\right)^{\gamma}(1-\varepsilon)^{\gamma} \Omega_{n-1}\left(1-A_{n-1}\right)^{\gamma} e^{-\delta \tau_{n-1}}\left[X_{\tau_{n-1}}^{\gamma} \mid \mathcal{H}_{\tau_{n-2}}\right] \\
& =D R_{n}\left(1-A_{n}\right)^{\gamma} R_{n-1}\left(1-A_{n-1}\right)^{\gamma} \ldots R_{1}\left(1-A_{1}\right)^{\gamma} X_{0}^{\gamma} \\
& =D X_{0}^{\gamma} \prod_{k=1}^{n} R_{k}\left(1-A_{k}\right)^{\gamma}=D X_{0}^{\gamma}\left[\left[e^{-\delta \bar{K}}(1-\varepsilon)^{\gamma} \Omega_{n}\right]^{1 / \nu}\right]^{n},
\end{aligned}
$$

as $1-A_{n}=R_{n}^{1 / \nu}=\left[(1-\varepsilon)^{\gamma} \Omega_{n} e^{-\delta \bar{K}}\right], \quad \forall n$. Since $\left[(1-\varepsilon)^{\gamma} \Omega_{n} e^{-\delta \bar{K}}\right]<$ $(1-\varepsilon)^{\gamma}$, for any feasible policy, then $\lim _{n \rightarrow \infty} E\left[e^{-\delta \tau_{n}} f\left(X_{\tau_{n}}\right)\right]=0$. Hence, condition (ii) of Lemma 3.3 is satisfied. By Lemma 3.3, the proof of Theorem 3.5 has been completed 


\section{Chapter 4}

\section{Portfolio Selection with Linear Transaction Costs}

\subsection{Introduction}

Chapter 4 is concerned with consumption and investment strategy for an investor who seeks to maximize the expected utility of consumption. The investor has available a riskless asset paying fixed interest rate and a risky asset with logarithmic Brownian motion price fluctuations. The objective is to maximize the expected discounted utility of consumption. The investor observes his/her current wealth and makes transaction at stopping times $\tau_{1}, \tau_{2}, \tau_{3}, \ldots$. The decision to transact can be made at any time based on all current information. For every transaction, the investor is charged a fraction of the current wealth as a management fee plus transaction costs which is a linear function of the amount of wealth transacted. 
The problem faced by the investor, as formulated in Chapter 2, is in a discrete-continuous time optimal control problem form. The optimal control problem for the investor is to choose optimal policy $(T, W, V, C)$ in a set of feasible policies $\mathcal{U}$, such that the value function is maximized. The main task of Chapter 4 is to find an optimal solution to that optimal control problem. Analysis in Chapter 4 is similar to that of Chapter 3. As in Chapter 3, we will solve the problem in two steps. In the first step, a deterministic continuous-time optimal control problem for consumption is solved, for a given $(T, W, V)$. In the second step, equipped with the optimal value function from the first step, a stochastic discrete-time optimal control problem is solved. The existence of an optimal consumption and investment selection is given.

The rest of Chapter 4 is organized as follows. Section 4.2 states the problem which is formulated in Chapter 2. In Section 4.3, we show that it is not optimal for the investor to withdraw more money than the amount needed for consumption. For a given $(T, W, V)$, the optimal consumption for the investor is solved. Section 4.4 is concerned with the derivation of optimal money withdrawals process and investment strategy for the risky security. This is done by deriving the optimal value function for fixed interval $T_{n}$ for all $\mathrm{n}$. This is one of the main features of Chapter 4 as well as this thesis. In Section 4.5, with optimal $(W, V, C)$ in hand, an equation satisfied by the transaction intervals is derived. Each optimal transaction interval infact satisfies a nonlinear equation which is not independent of total wealth at the beginning of that interval. A solution to that equation exists and is optimal. 


\subsection{Statement of the Problem}

The model is as in Chapter 2, with linear transaction costs. The transaction costs function $\Psi$ takes the form $\Psi\left(W_{\tau_{n}}\right)=a W_{\tau_{n}}+b$, with $a \in(0,1)$, and $b \geq 0$. The money holding at any time $\mathrm{t}$ is given by

$$
M_{t}=\sum_{\left\{n: \tau_{n} \leq t\right\}}\left[(1-a) W_{\tau_{n}}-b\right]-\int_{0}^{t} C_{s} d s .
$$

Definition 4.1 Let $\mathcal{U}=\mathcal{T} \times \mathcal{W} \times \mathcal{V} \times \mathcal{C}$ be the set of all budget feasible policies, where $\mathcal{T}, \mathcal{W}, \mathcal{V}, \mathcal{C}$ are defined as in Chapter 2. Then the optimal control problem for the investor is to maximize the value function

$$
U\left(X_{0}\right) \equiv \max _{(T, W, V, C) \in \mathcal{U}} E\left[\int_{0}^{\infty} e^{-\delta t} u\left(C_{t}\right) d t\right]
$$

subject to, for $n=1,2,3, \ldots$

$$
X_{\tau_{n+1}}=(1-\varepsilon)\left[X_{\tau_{n}}-W_{\tau_{n}}\right] e^{r T_{n}}+V_{\tau_{n}}\left[\Gamma_{n+1}-e^{r T_{n}}\right],
$$

and $X_{\tau_{n+1}} \geq 0$, and $M_{t} \geq 0$.

\subsection{Consumption Strategy}

Section 4.3 is mainly concerned with the optimal consumption strategy. As it is the case in Section 3.3, it is not optimal for the investor to take out more money from his/her portfolio than needed for his/her consumption. This claim is proved in the following Lemma.

Lemma 4.1 Let the value function $U$ be defined as in (4.2), and the transaction costs function $\Psi$ is of the form $\Psi\left(W_{\tau_{n}}\right)=a W_{\tau_{n}}+b$. Then the 
optimal policy $(T, W, V, C)$ must satisfy for all $n=1,2,3, \ldots$

$$
\int_{\tau_{n}}^{\tau_{n+1}} C_{t} d t=(1-a) W_{\tau_{n}}-b
$$

Proof : The proof is similar to those of Lemma 3.1. Let a feasible policy $(T, W, V, C)$ be an optimal policy. Suppose that there exists an interval $T_{j}=\tau_{j+1}-\tau_{j}$ such that $d=(1-a) W_{\tau_{j}}-b-\int_{\tau_{j}}^{\tau_{j+1}} C_{t} d t$. Because there exists a riskless security with a positive interest rate, then the investor will be better off if he/she invests the amount $d$ in the riskless security during the interval $T_{j}$, and consumes the interest income $d\left(e^{r T_{j}}-1\right)$ in the next interval. In other words, the optimal policy $(T, W, V, C)$ is dominated by a feasible policy $(T, \bar{W}, V, \bar{C})$ which is defined by

$$
\begin{gathered}
\bar{W}_{\tau_{j}}=\frac{1}{1-a}\left[\int_{\tau_{j}}^{\tau_{j+1}} C_{t} d t+b\right], \\
\bar{W}_{\tau_{j+1}}=W_{\tau_{j+1}}+d e^{r T_{j}}>W_{\tau_{j+1}}, \\
\bar{C}_{t}=C_{t}+\frac{1}{T_{j+1}} d\left(e^{r T_{j}}-1\right)>C_{t}, \quad t \in\left[\tau_{j+1}, \tau_{j+2}\right), \\
\bar{C}_{t}=C_{t}, \quad \bar{W}_{\tau_{j}}=W_{\tau_{j}}, \quad \text { otherwise. }
\end{gathered}
$$

But $(T, W, V, C)$ is optimal. Hence contradiction. Therefore, for all $n$,

$$
\int_{\tau_{n}}^{\tau_{n+1}} C_{t} d t \geq(1-a) W_{\tau_{n}}-b .
$$

On the other hand, all expenditures must be financed from the stock of money. Then for all $n, \sum_{i}^{n} \int_{\tau_{i}}^{\tau_{i+1}} C_{t} d t \geq \sum_{i}^{n}(1-a) W_{\tau_{i}}-b$. Therefore, for all $n$, an optimal policy $(T, W, V, C)$ must satisfy

$$
\int_{\tau_{n}}^{\tau_{n+1}} C_{t} d t=(1-a) W_{\tau_{n}}-b
$$


Corollary 4.1 By the definition of money holding $M_{t}$, then

$$
M_{\tau_{n}}=(1-a) W_{\tau_{n}}-b, \quad n=1,2,3, \ldots
$$

Therefore, the optimal control problem (4.2)-( 4.3) is equivalent to the optimal control problem :

$$
U\left(X_{0}\right)=\max _{(T, W, V, C) \in \mathcal{U}} E\left[\int_{0}^{\infty} e^{-\delta t} u\left(C_{t}\right) d t\right]
$$

subject to, for $n=1,2,3, \ldots$

$$
\begin{gathered}
\int_{\tau_{n}}^{\tau_{n+1}} C_{t} d t=(1-a) W_{\tau_{n}}-b, \\
X_{\tau_{n+1}}=(1-\varepsilon)\left[X_{\tau_{n}}-W_{\tau_{n}}\right] e^{r T_{n}}+V_{\tau_{n}}\left[\Gamma_{n+1}-e^{r T_{n}}\right] \geq 0 .
\end{gathered}
$$

As in Section 3.3, the value function $U$ may be re-written as

$$
U\left(X_{0}\right)=E\left[\sum_{n=1}^{\infty} e^{-\delta \tau_{n}} \int_{\tau_{n}}^{\tau_{n+1}} e^{-\delta\left(t-\tau_{n}\right)} u\left(C_{t}\right) d t\right] .
$$

The optimal control problem (4.5)-( 4.7) then will be solved in two steps. In the first step, a deterministic control problem for consumption between intervals is solved, for any given $(T, W, V)$. The objective function for this control problem is denoted by $J$. In the second step, a discrete-time stochastic optimal control is solved, using the optimal value function from the first step.

The deterministic control problem for which the investor needs to solve in the first step is maximize the objective function

$$
J(t, Z)=\int_{0}^{t} \exp (-\delta s) u\left(C_{s}\right) d s
$$

over $\left\{C_{s}: 0 \leq s \leq t\right\}$, subject to :

$$
\int_{0}^{t} C_{t} d t \leq Z
$$


Lemma 4.2 The optimal value function for the above optimal control problem satisfies

$$
J(t, Z)=\left(\frac{1-\gamma}{\delta}\right)^{1-\gamma}\left[1-\exp \left(-\frac{\delta}{1-\gamma} t\right)\right]^{1-\gamma} \frac{1}{\gamma} Z^{\gamma}
$$

The proof of Lemma 4.2 is similar to those of Lemma 3.2 and hence, is omitted. By Lemma 4.2 and Corollary 4.1 then

$$
J\left(T_{n}, M_{\tau_{n}}\right)=\left[1-\exp \left(-\frac{\delta}{1-\gamma} T_{n}\right)\right]^{1-\gamma} \frac{1}{\gamma}\left[(1-a) W_{\tau_{n}}-b\right]^{\gamma},
$$

with $((1-\gamma) / \delta)^{1-\gamma}$ has been left out.

Let $Q_{n}=1-\exp \left(-\frac{\delta}{\nu} T_{n}\right)$, where $\nu=1-\gamma$. Then the modified optimal control problem is given by

$$
U\left(X_{0}\right)=\max _{\{T \in \mathcal{T}, W \in \mathcal{W}, V \in \mathcal{V}\}} E\left[\sum_{n=1}^{\infty} e^{-\delta \tau_{n}} Q_{n}^{\nu} \frac{1}{\gamma}\left[(1-a) W_{\tau_{n}}-b\right]^{\gamma}\right]
$$

subject to, for $n=1,2,3, \ldots$

$$
X_{\tau_{n+1}}=(1-\varepsilon)\left[X_{\tau_{n}}-W_{\tau_{n}}\right] e^{r T_{n}}+V_{\tau_{n}}\left[\Gamma_{n+1}-e^{r T_{n}}\right] \geq 0 .
$$

By the application of Bellman principle on $U$, then for $n=1,2,3, \ldots$

$$
U\left(X_{\tau_{n}}\right)=\max _{\left\{T_{n}, W_{\tau_{n}}, V_{\tau_{n}}\right\}}\left\{Q_{n}^{\nu} \frac{1}{\gamma}\left[(1-a) W_{\tau_{n}}-b\right]^{\gamma}+e^{-\delta T_{n}} E\left[U\left(X_{\tau_{n+1}}\right) \mid \mathcal{H}_{\tau_{n}}\right]\right\}
$$

subject to, for $n=1,2,3, \ldots$

$$
X_{\tau_{n+1}}=(1-\varepsilon)\left[X_{\tau_{n}}-W_{\tau_{n}}\right] e^{r T_{n}}+V_{\tau_{n}}\left[\Gamma_{n+1}-e^{r T_{n}}\right] .
$$

Lemma 4.3 Let $\bar{Q}(n)=\left[1-\exp \left(-\frac{\delta}{\nu} T_{n}\right)\right]^{\nu}$. Suppose that $f$ is a realvalued function on $[0, \infty)$ satisfying the two conditions : 
(i) For all $n=1,2,3, \ldots$,

$$
f\left(X_{\tau_{n}}\right)=\max _{\left(T_{n}, W_{\tau_{n}}, V_{\tau_{n}}\right)}\left\{\bar{Q}(n) \frac{1}{\gamma}\left[(1-a) W_{\tau_{n}}-b\right]^{\gamma}+E\left[e^{-\delta T_{n}} f\left(X_{\tau_{n+1}}\right)\right] \mid \mathcal{H}_{\tau_{n}}\right\} .
$$

(ii) For any feasible policy,

$$
\lim _{n \rightarrow \infty} E\left[e^{-\delta \tau_{n}} f\left(X_{\tau_{n}}\right)\right]=0 .
$$

If $\left(T^{*}, W^{*}, V^{*}\right)$ achieves the maximum in (4.12) for all $n$ then $f$ is the value function for the control problem (4.10), and $\left(T^{*}, W^{*}, V^{*}\right)$ is an optimal policy.

Proof : Let $n=1$, to begin with, that is $\tau_{1}=0$. Then

$$
\begin{aligned}
f\left(X_{0}\right) & =\max _{\left(T_{1}, W_{0}, V_{0}\right)}\left\{\bar{Q}(1) \frac{1}{\gamma}\left[(1-a) W_{0}-b\right]^{\gamma}+E\left[e^{-\delta T_{1}} f\left(X_{\tau_{2}}\right)\right]\right\} \\
& \geq \bar{Q}(1) \frac{1}{\gamma}\left[(1-a) W_{0}-b\right]^{\gamma}+e^{-\delta T_{1}} E\left[f\left(X_{\tau_{2}}\right)\right]
\end{aligned}
$$

for any feasible $T_{1}, W_{0}, V_{0}$. By induction, for any $(T, W, V) \in \mathbf{T} \times \mathbf{W} \times \mathbf{V}$ then

$$
f\left(X_{0}\right) \geq E\left[\sum_{i=1}^{n} e^{-\delta \tau_{i}} \bar{Q}(i) \frac{1}{\gamma}\left[(1-a) W_{\tau_{i}}-b\right]^{\gamma}+e^{-\delta \tau_{n+1}} f\left(X_{\tau_{n+1}}\right)\right] .
$$

Let $n \rightarrow \infty$, it follows by condition (ii) of Lemma 4.3 that

$$
f\left(X_{0}\right) \geq E\left[\sum_{n=1}^{\infty} e^{-\delta \tau_{n}} \bar{Q}(n) \frac{1}{\gamma}\left[(1-a) W_{\tau_{n}}-b\right]^{\gamma}\right] .
$$

This holds for an arbitrary feasible policy $(T, V, W)$. Hence,

$$
f\left(X_{0}\right) \geq U\left(X_{0}\right)
$$

On the other hand, $U\left(X_{0}\right) \geq f\left(X_{0}\right)$ by the definition of $U\left(X_{0}\right)$. Henceforth, $f\left(X_{0}\right)=U\left(X_{0}\right)$, and consequently $\left(T^{*}, W^{*}, V^{*}\right)$ is optimal 


\subsection{Investment Strategies}

Section 4.4 is mainly concerned with the derivation of optimal value function for the problem (4.10) subject to (4.11). A closed form for the optimal utility function, as well as the optimal withdrawal and investment strategies, are derived. The analysis in Section 4.4 is similar to that in Section 3.4.

Theorem 4.1 Let $T_{n}$ be fixed for all $n=1,2,3, \ldots$. Then the optimal value function and unique solution to the problem (4.10)-(4.11) is given by

$$
U\left(X_{\tau_{n}}\right)=Q_{n}^{\nu} A_{n}^{-\nu} \frac{1}{\gamma}\left(X_{\tau_{n}}-Y_{n}\right)^{\gamma}
$$

and the optimal withdrawal and investment strategies are given by

$$
\begin{aligned}
W_{\tau_{n}} & =\frac{1}{1-a} F_{n}\left(X_{\tau_{n}}-Y_{n}\right)+\frac{b}{1-a} \\
V_{\tau_{n}} & =(1-\varepsilon)\left(1-F_{n}\right)\left(X_{\tau_{n}}-Y_{n}\right) \pi_{n}
\end{aligned}
$$

where $A_{n}, F_{n}$ and $Y_{n}$ are defined by recurrence relationships :

$$
\begin{aligned}
A_{n} & =\frac{A_{n+1} Q_{n}\left[A_{n+1} Q_{n}(1-a)^{1 / \nu}+Q_{n+1} R_{n}^{1 / \nu}\right]^{\gamma / \nu}}{\left[A_{n+1} Q_{n}(1-a)^{\gamma / \nu}+Q_{n+1} R_{n}^{1 / \nu}\right]^{1 / \nu}} \\
F_{n} & =\frac{A_{n+1} Q_{n}(1-a)^{1 / \nu}}{A_{n+1} Q_{n}(1-a)^{1 / \nu}+Q_{n+1} R_{n}^{1 / \nu}} \\
Y_{n} & =b+a W_{\tau_{n}}+(1-\varepsilon)^{-1} e^{-r T_{n}} Y_{n+1}
\end{aligned}
$$

with $R_{n}=(1-\varepsilon)^{\gamma} e^{-\delta T_{n}} \Omega_{n}$, and where $\Omega_{n}$ and $\pi_{n}$ are defined by the optimization problem :

$$
\begin{aligned}
\Omega_{n} & \equiv E\left(\left[e^{r T_{n}}+\pi_{n}\left(\Gamma_{n+1}-e^{r T_{n}}\right)\right]^{\gamma}\right) \\
& \equiv \sup _{\{0 \leq \pi \leq 1\}} E\left(\left[e^{r T_{n}}+\pi\left(\Gamma_{n+1}-e^{r T_{n}}\right)\right]^{\gamma}\right),
\end{aligned}
$$


Remark 4.1 Since $C \geq 0$, then (4.15) implies that $W_{\tau_{n}} \geq b /(1-a)$, and hence $X_{\tau_{n}} \geq Y_{n}$.

Proof of Theorem : The principle of the proof is similar to that of Theorem 3.1. Let denote the right-hand side of relation ( 4.10) by $S\left(X_{\tau_{n}}\right)$ upon inserting relation ( 4.14$)$ for $U\left(X_{\tau_{n}}\right)$. This implies that $S\left(X_{\tau_{n}}\right)$ may be written as

$$
\begin{aligned}
S\left(X_{\tau_{n}}\right) & =\max _{\left\{W_{\tau_{n}}, V_{\tau_{n}}\right\}}\left\{Q_{n}^{\nu} \frac{1}{\gamma}\left[(1-a) W_{\tau_{n}}-b\right]^{\gamma}\right. \\
& \left.+Q_{n+1}^{\nu} A_{n+1}^{-\nu} e^{-\delta T_{n}} E\left[\frac{1}{\gamma}\left(X_{\tau_{n+1}}-Y_{n+1}\right)^{\gamma} \mid \mathcal{H}_{\tau_{n}}\right]\right\} .
\end{aligned}
$$

Let $Y_{n}$ be defined by recurrence relationship

$$
Y_{n}=a W_{\tau_{n}}+b+(1-\varepsilon)^{-1} e^{-r T_{n}} Y_{n+1}
$$

Then the total wealth process $X_{\tau_{n+1}}$ as given by relation (4.11) may be written as

$$
\begin{aligned}
X_{\tau_{n+1}} & =(1-\varepsilon)\left[X_{\tau_{n}}-Y_{n}-\left((1-a) W_{\tau_{n}}-b\right)\right] e^{r T_{n}} \\
& +V_{\tau_{n}}\left(\Gamma_{n+1}-e^{r T_{n}}\right)+Y_{n+1} .
\end{aligned}
$$

Therefore, $S\left(X_{\tau_{n}}\right)$ may be written as

$$
\begin{aligned}
S\left(X_{\tau_{n}}\right) & =\max _{\left\{W_{\tau_{n}}, V_{\tau_{n}}\right\}}\left\{Q_{n}^{\nu} \frac{1}{\gamma}\left((1-a) W_{\tau_{n}}-b\right)^{\gamma}+A_{n+1}^{-\nu} Q_{n+1}^{\nu} e^{-\delta T_{n}}(1-\varepsilon)^{\gamma}\right. \\
& \left.\times E\left[\frac{1}{\gamma}\left(\left[X_{\tau_{n}}-Y_{n}-\left((1-a) W_{\tau_{n}}-b\right)\right] e^{r T_{n}}+V_{\tau_{n}}\left(\Gamma_{n+1}-e^{r T_{n}}\right)\right)^{\gamma}\right]\right\},
\end{aligned}
$$

subject to :

$$
W_{\tau_{n}} \geq \frac{b}{1-a}
$$




$$
\begin{gathered}
P\left\{X_{\tau_{n+1}}-Y_{n+1} \geq 0\right\}=1 \\
V_{\tau_{n}} \geq 0
\end{gathered}
$$

Note that, for $X_{\tau_{n}}-Y_{n}-\left((1-a) W_{\tau_{n}}-b\right)>0$, by re-arrangement, the total wealth process $X_{\tau_{n+1}}$ of (4.22) may be re-written as

$X_{\tau_{n+1}}=(1-\varepsilon)\left[X_{\tau_{n}}-Y_{n}-\left((1-a) W_{\tau_{n}}-b\right)\right]\left[e^{r T_{n}}+I_{n}\left(\Gamma_{n+1}-e^{r T_{n}}\right)\right]+Y_{n+1}$,

where $I_{n}$ is defined by

$$
I_{n}=\frac{V_{\tau_{n}}}{(1-\varepsilon)\left[X_{\tau_{n}}-Y_{n}-\left((1-a) W_{\tau_{n}}-b\right)\right]} .
$$

Hence,

$$
\begin{aligned}
S\left(X_{\tau_{n}}\right) & =\max _{\left\{W_{\tau_{n}}, V_{\tau_{n}}\right\}}\left\{Q_{n}^{\nu} \frac{1}{\gamma}\left[(1-a) W_{\tau_{n}}-b\right]^{\gamma}+A_{n+1}^{-\nu} Q_{n+1}^{\nu} e^{-\delta T_{n}}\right. \\
& \times(1-\varepsilon)^{\gamma}\left[X_{\tau_{n}}-Y_{n}-\left((1-a) W_{\tau_{n}}-b\right)\right]^{\gamma} \\
& \left.\times E\left[\frac{1}{\gamma}\left[e^{r T_{n}}+I_{n}\left(\Gamma_{n+1}-e^{r T_{n}}\right)\right]^{\gamma}\right]\right\} .
\end{aligned}
$$

To prevent the problem being trivial, the following condition is imposed :

$$
P\left\{\theta\left(\Gamma_{n+1}-e^{r T_{n}}\right)<0\right\}>0, \quad \theta>0 .
$$

Then it follows that $P\left\{X_{\tau_{n+1}}-Y_{n+1} \geq 0\right\}=1$, if and only if :

$$
X_{\tau_{n}}-Y_{n}-\left[(1-a) W_{\tau_{n}}-b\right]=0 \text { and } V_{\tau_{n}}=0
$$

or,

$$
X_{\tau_{n}}-Y_{n}-\left[(1-a) W_{\tau_{n}}-b\right]>0,
$$

and

$$
P\left\{e^{r T_{n}}+I_{n}\left(\Gamma_{n+1}-e^{r T_{n}}\right) \geq 0\right\}=1 .
$$


Under feasibility with respect to $P\left\{X_{\tau_{n+1}}-Y_{n+1} \geq 0\right\}=1$, then

$$
S\left(X_{\tau_{n}}\right)=\max \left\{Q_{n}^{\nu} \frac{1}{\gamma}\left[(1-a) W_{\tau_{n}}-b\right]^{\gamma}, \bar{S}\left(X_{\tau_{n}}\right)\right\},
$$

where

$$
\begin{aligned}
& \bar{S}\left(X_{\tau_{n}}\right)=\sup _{\left\{W_{\tau_{n}}, V_{\tau_{n}}\right\}}\left\{Q_{n}^{\nu} \frac{1}{\gamma}\left[(1-a) W_{\tau_{n}}-b\right]^{\gamma}+A_{n+1}^{-\nu} Q_{n+1}^{\nu} e^{-\delta T_{n}}\right. \\
& \left.\times(1-\varepsilon)^{\gamma}\left[X_{\tau_{n}}-Y_{n}-\left((1-a) W_{\tau_{n}}-b\right)\right]^{\gamma} E\left[\frac{1}{\gamma}\left(e^{r T_{n}}+I_{n}\left(\Gamma_{n+1}-e^{r T_{n}}\right)\right)^{\gamma}\right]\right\},
\end{aligned}
$$

subject to (4.23), (4.28), (4.29) and $I_{n} \geq 0$, since this is equivalent to ( 4.25$)$ in view of (4.29).

The expectation factor in relation (4.31) may be denoted by $f\left(I_{n}\right)$, where $f$ is defined by

$$
f(\pi)=E\left[u\left(\exp \left(r T_{n}\right)+\pi\left(\Gamma_{n+1}-\exp \left(r T_{n}\right)\right)\right)\right]
$$

with $u(C)=\frac{1}{\gamma} C^{\gamma}, \quad \gamma \in(0,1)$. Therefore,

$$
\begin{aligned}
\bar{S}\left(X_{\tau_{n}}\right) & =\max _{\left\{W_{\tau_{n}}, V_{\tau_{n}}\right\}}\left\{Q_{n}^{\nu} \frac{1}{\gamma}\left[(1-a) W_{\tau_{n}}-b\right]^{\gamma}+A_{n+1}^{-\nu} Q_{n+1}^{\nu}\right. \\
& \left.\times(1-\varepsilon)^{\gamma} e^{-\delta T_{n}}\left[X_{\tau_{n}}-Y_{n}-\left((1-a) W_{\tau_{n}}-b\right)\right]^{\gamma} f\left(I_{\tau_{n}}\right)\right\} .
\end{aligned}
$$

According to Lemma 3.4 and relation (4.20), the maximum of of $f\left(I_{n}\right)$, subject to $I_{n} \geq 0$, and ( 4.29) is given by $\frac{1}{\gamma} \Omega_{n}$. Then by Lemma 3.4, we have $I_{n}=\pi_{n}$. Therefore,

$$
V_{\tau_{n}}=(1-\varepsilon)\left[X_{\tau_{n}}-Y_{n}-\left((1-a) W_{\tau_{n}}-b\right)\right] \pi_{n}
$$

is optimal and unique for every $W_{\tau_{n}}$ which satisfies ( 4.23) and (4.28) when ( 4.29 ) holds. It can be shown that it is also optimal when relation (4.27) holds. 
Note that the second term of $\bar{S}\left(X_{\tau_{n}}\right)$ is always nonnegative. This implies that

$$
\left.\bar{S}\left(X_{\tau_{n}}\right) \geq Q_{n}^{\nu} \frac{1}{\gamma}(1-a) W_{\tau_{n}}-b\right)^{\gamma}
$$

Therefore $S\left(X_{\tau_{n}}\right)$ as given by relation (4.30) reduces to

$$
S\left(X_{\tau_{n}}\right)=\max _{\left\{W_{\tau_{n}}\right\}} S^{W_{\tau_{n}}}\left(X_{\tau_{n}}\right)
$$

where

$$
\begin{aligned}
S^{W_{\tau_{n}}}\left(X_{\tau_{n}}\right) & =Q_{n}^{\nu} \frac{1}{\gamma}\left[(1-a) W_{\tau_{n}}-b\right]^{\gamma}+A_{n+1}^{-\nu} Q_{n+1}^{\nu} R_{n} \\
& \times \frac{1}{\gamma}\left[X_{\tau_{n}}-Y_{n}-\left((1-a) W_{\tau_{n}}-b\right)\right]^{\gamma}
\end{aligned}
$$

with $R_{n}=(1-\varepsilon)^{\gamma} e^{-\delta T_{n}} \Omega_{n}$. Since the utility function $u$ is strictly concave and $u^{\prime}(0)=\infty$, then $S^{W_{\tau_{n}}}\left(X_{\tau_{n}}\right)$ is differentiable with respect to $W_{\tau_{n}}$.

Differentiation of $S^{W_{\tau_{n}}}$ with respect to $W_{\tau_{n}}$ results in

$$
\begin{aligned}
& \frac{d S^{W_{\tau_{n}}}}{d W_{\tau_{n}}}=Q_{n}^{\nu}\left[(1-a) W_{\tau_{n}}-b\right]^{-\nu}(1-a)-A_{n+1}^{-\nu} Q_{n+1}^{\nu} R_{n} \\
& \quad \times\left[X_{\tau_{n}}-Y_{n}-\left((1-a) W_{\tau_{n}}-b\right)\right]^{-\nu}\left(\frac{d Y_{n}}{d W_{\tau_{n}}}+(1-a)\right) .
\end{aligned}
$$

From relation ( 4.21) we have $d Y_{n} / d W_{\tau_{n}}=a$. By setting $d S^{W_{\tau_{n}}} / d W_{\tau_{n}}=0$, and re-arranging all terms, result in

$$
\begin{aligned}
& (1-a) W_{\tau_{n}}-b \\
& \quad=A_{n+1} Q_{n} Q_{n+1}^{-1} R_{n}^{-1 / \nu}(1-a)^{1 / \nu}\left[X_{\tau_{n}}-Y_{n}-\left((1-a) W_{\tau_{n}}-b\right)\right] .
\end{aligned}
$$

Let $F_{n}$ be defined by

$$
F_{n}=\frac{A_{n+1} Q_{n}(1-a)^{1 / \nu}}{A_{n+1} Q_{n}(1-a)^{1 / \nu}+Q_{n+1} R_{n}^{1 / \nu}} .
$$


Then the optimal withdrawal process $W_{\tau_{n}}$ may be expressed as

$$
W_{\tau_{n}}=\frac{F_{n}}{1-a}\left(X_{\tau_{n}}-Y_{n}\right)+\frac{b}{1-a} .
$$

By insertion of ( 4.34) into ( 4.32 ) results in the investment process $V_{\tau_{n}}$ is in the form

$$
V_{\tau_{n}}=(1-\varepsilon)\left(1-F_{n}\right)\left(X_{\tau_{n}}-Y_{n}\right) \pi_{n}
$$

Substitution of ( 4.34) into (4.33) results in

$$
\begin{aligned}
S\left(X_{\tau_{n}}\right) & =Q_{n}^{\nu} F_{n}^{\gamma} \frac{1}{\gamma}\left(X_{\tau_{n}}-Y_{n}\right)^{\gamma}+A_{n+1}^{-\nu} Q_{n+1}^{\nu} R_{n} \\
& \times \frac{1}{\gamma}\left(X_{\tau_{n}}-Y_{n}-F_{n}\left(X_{\tau_{n}}-Y_{n}\right)\right)^{\gamma} \\
& =Q_{n}^{\nu} A_{n}^{-\nu} \frac{1}{\gamma}\left(X_{\tau_{n}}-Y_{n}\right)^{\gamma}\left[A_{n}^{\nu} F_{n}^{\gamma}\right. \\
& \left.+A_{n}^{\nu} Q_{n}^{-\nu} A_{n+1}^{-\nu} Q_{n+1}^{\nu} R_{n}\left(1-F_{n}\right)^{\gamma}\right] .
\end{aligned}
$$

Therefore, $S\left(X_{\tau_{n}}\right)=U\left(X_{\tau_{n}}\right)$ if and only if

$$
A_{n}^{\nu} F_{n}^{\gamma}+A_{n}^{\nu} Q_{n}^{-\nu} A_{n+1}^{-\nu} Q_{n+1}^{\nu} R_{n}\left(1-F_{n}\right)^{\gamma}=1 .
$$

Relation $(\star)$ holds if and only if

$$
\begin{aligned}
& {\left[A_{n+1} Q_{n}(1-a)^{1 / \nu}+Q_{n+1} R_{n}^{1 / \nu}\right]^{\gamma}} \\
& \quad=A_{n}^{\nu} A_{n+1}^{-\nu} Q_{n}^{-\nu}\left[A_{n+1} Q_{n}(1-a)^{\gamma / \nu}+Q_{n+1} R_{n}^{1 / \nu}\right]
\end{aligned}
$$

This implies that relation $(\star)$ holds if and only if

$$
A_{n}=\frac{A_{n+1} Q_{n}\left[A_{n+1} Q_{n}(1-a)^{1 / \nu}+Q_{n+1} R_{n}^{1 / \nu}\right]^{\gamma / \nu}}{\left[A_{n+1} Q_{n}(1-a)^{\gamma / \nu}+Q_{n+1} R_{n}^{1 / \nu}\right]^{1 / \nu}} .
$$

Therefore, the proof of Theorem 4.1 has been completed

The following remark is in line with Corollary 3.2. 
Remark 4.2 Let the optimal control problem be defined by problem (4.10)(4.11). Furthermore, let $T_{n}$ be fixed for $n=1,2,3, \ldots$. Then $F_{n}$ as given by (4.18) has a property such that either $F_{n} \geq Q_{n}\left(1-R_{n}^{1 / \nu}\right)$, or $F_{n}<Q_{n}\left(1-R_{n}^{1 / \nu}\right)$.

Remark 4.3 Suppose that $A_{n+1}$ satisfying relation (4.18). If $F_{n}$ as given by relation ( 4.18) is such that $F_{n} \geq Q_{n}\left(1-R_{n}^{1 / \nu}\right)$, then $A_{n+1}$ is such that

$$
\frac{A_{n+1}}{Q_{n+1}} \geq \frac{\left(1-R_{n}^{1 / \nu}\right) R_{n}^{1 / \nu}}{(1-a)^{1 / \nu}}
$$

Proof : By applying $F_{n} \geq Q_{n}\left(1-R_{n}^{1 / \nu}\right)$ in relation (4.18), and arranging the terms, then $A_{n+1}$ satisfy

$$
\begin{aligned}
\frac{A_{n+1}}{Q_{n+1}} & =\frac{F_{n} R_{n}^{1 / \nu}}{Q_{n}\left(1-F_{n}\right)(1-a)^{1 / \nu}} \\
& \geq \frac{Q_{n}\left(1-R_{n}^{1 / \nu}\right) R_{n}^{1 / \nu}}{Q_{n}\left(1-F_{n}\right)(1-a)^{1 / \nu}} \\
& \geq \frac{\left(1-R_{n}^{1 / \nu}\right) R_{n}^{1 / \nu}}{\left[1-Q_{n}\left(1-R_{n}^{1 / \nu}\right)\right](1-a)^{1 / \nu}} .
\end{aligned}
$$

Since $Q_{n}$ and $R_{n}$ both are nonnegatives and less than one, then $\left[1-Q_{n}\left(1-R_{n}^{1 / \nu}\right)\right] \leq 1$. This implies that

$$
\frac{\left(1-R_{n}^{1 / \nu}\right) R_{n}^{1 / \nu}}{\left[1-Q_{n}\left(1-R_{n}^{1 / \nu}\right)\right](1-a)^{1 / \nu}} \geq \frac{\left(1-R_{n}^{1 / \nu}\right) R_{n}^{1 / \nu}}{(1-a)^{1 / \nu}} .
$$

Hence, $\quad \frac{A_{n+1}}{Q_{n+1}} \geq \frac{\left(1-R_{n}^{1 / \nu}\right) R_{n}^{1 / \nu}}{(1-a)^{1 / \nu}}$ 
Remark 4.4 If at any $\tau_{n}, \quad W_{\tau_{n}}=b /(1-a)$, then the implications are as follows :

1. $Y_{n}=X_{\tau_{n}}$, by $(4.15)$

2. $T_{n}$ can be computed from (4.19) as

$$
0<T_{n}=\frac{1}{r} \ln \left[\frac{X_{\tau_{n+1}}}{\left(X_{\tau_{n}}-\frac{b}{1-a}\right)(1-\varepsilon)}\right]<\infty .
$$

3. $U\left(X_{\tau_{n}}\right)=0$, implying by (4.2) that $C_{t}=0$, almost every where for $t \geq \tau_{n}$. This can occur only for utility function which satisfy $u(0)=0$.

4. Case 2 and 3 imply that $W_{\tau_{n+1}}>b /(1-a)$ always in the case of infinite-time forizon.

Corollary 4.2 It is possible that $W_{\tau_{n}}=b /(1-a)$ in which case

$$
T_{n}=\frac{1}{r} \ln \left[\frac{X_{\tau_{n+1}}}{\left(X_{\tau_{n}}-\frac{b}{1-a}\right)(1-\varepsilon)}\right] .
$$

This imply that $W_{\tau_{n}}>b /(1-a)$ if the specified $T_{n}$ are not given by (4.36). 


\subsection{Optimal Transaction Intervals}

The aim of this section is to find the optimal transaction times for an investor who has the optimal control problem as defined by the optimal control problem (4.10)-( 4.11). Since finding the optimal transaction times is equivalent to finding the optimal transaction intervals, then this section is devoted to finding the optimal transaction intervals $T_{n}$, for $n=1,2,3, \ldots$ This is done by establishing an equation satisfied by the interval $T_{n}$, and later, by confirming a solution to that equation which is the optimal choice for $T_{n}$.

Recall the value function $U\left(X_{\tau_{n}}\right)$ as defined by relation (4.10), which is given by

$$
U\left(X_{\tau_{n}}\right)=\max _{\left\{T_{n}, W_{\tau_{n}}, V_{\tau_{n}}\right\}}\left\{Q_{n}^{\nu} \frac{1}{\gamma}\left[(1-a) W_{\tau_{n}}-b\right]^{\gamma}+e^{-\delta T_{n}} E\left[U\left(X_{\tau_{n+1}}\right) \mid \mathcal{H}_{\tau_{n}}\right]\right\}
$$

By taking the first derivative of the value function $U$ with respect to $W_{\tau_{n}}$, $V_{\tau_{n}}$, and $T_{n}$, respectively, and setting each of these derivatives equals to zero, then result in the following necessary conditions for $U\left(X_{\tau_{n}}\right)$, for $n=1,2,3, \ldots$

$$
\begin{gathered}
Q_{n}^{\nu}\left[(1-a) W_{\tau_{n}}-b\right]^{-\nu}(1-a)=(1-\varepsilon) e^{-(\delta-r) T_{n}} E\left[U^{\prime}\left(X_{\tau_{n+1}}\right) \mid \mathcal{H}_{\tau_{n}}\right] \\
E\left[\left(\Gamma_{n+1}-e^{r T_{n}}\right) U^{\prime}\left(X_{\tau_{n+1}}\right) \mid \mathcal{H}_{\tau_{n}}\right]=0 \\
\delta e^{-\frac{\delta}{\nu} T_{n}} Q_{n}^{-\gamma} \frac{1}{\gamma}\left[(1-a) W_{\tau_{n}}-b\right]^{\gamma}= \\
e^{-\delta T_{n}}\left\{\delta E\left[U\left(X_{\tau_{n+1}}\right) \mid \mathcal{H}_{\tau_{n}}\right]-\frac{\partial E\left[U\left(X_{\tau_{n+1}}\right) \mid \mathcal{H}_{\tau_{n}}\right]}{\partial T_{n}}\right\} .
\end{gathered}
$$


Applications of Itós ${ }^{\prime}$ formula ${ }^{1}$ on the value function $U$, results in

$$
U\left(X_{\tau_{n+1}}\right)-U\left(X_{\tau_{n}}\right)=\int_{\tau_{n}+}^{\tau_{n+1}}\left[U^{\prime}\left(X_{t}\right) d X_{t}+\frac{1}{2} U^{\prime \prime}\left(X_{t}\right) d X_{t}^{2}\right] .
$$

From the definition of the total wealth process $X_{\tau_{n}}$ as given by (4.9) results in, for any $t \in\left[\tau_{n}, \tau_{n+1}\right)$,

$$
X_{t}=(1-\varepsilon)\left[X_{\tau_{n}}-W_{\tau_{n}}\right] e^{r\left(t-\tau_{n}\right)}+V_{\tau_{n}}\left(\Gamma_{t}-e^{r\left(t-\tau_{n}\right)}\right),
$$

with

$$
\Gamma_{t}=\exp \left[\left(\alpha-\frac{1}{2} \sigma^{2}\right)\left(t-\tau_{n}\right)+\sigma\left(B_{t}-B_{\tau_{n}}\right)\right]
$$

Then the following holds for $t \in\left[\tau_{n}, \tau_{n+1}\right]$ :

$$
d X_{t}=\left[r\left(X_{t}-G_{t}\right)+\alpha G_{t}\right] d t+\sigma G_{t} d B_{t}, \text { and } d X_{t}^{2}=\sigma^{2} G_{t}^{2} d t
$$

with $G_{t}$ is given by $G_{t} \equiv V_{\tau_{n}} \Gamma_{t}$. By substitutions of $d X_{t}$ and $d X_{t}^{2}$ into (4.38), then we have the following

$$
\begin{aligned}
U\left(X_{\tau_{n+1}}\right)-U\left(X_{\tau_{n}}\right) & =\int_{\tau_{n}+}^{\tau_{n+1}}\left\{\left[r\left(X_{t}-G_{t}\right)+\alpha V_{t}\right] U^{\prime}\left(X_{t}\right) d t\right. \\
& \left.+\frac{1}{2} \sigma^{2} G_{t}^{2} U^{\prime \prime}\left(X_{t}\right) d t+\sigma G_{t} U^{\prime}\left(X_{t}\right) d B_{t}\right\}
\end{aligned}
$$

Let processes $\left\{Z_{t}\right\}$ be defined as

$$
Z_{t}=\int_{\tau_{n}}^{t} \sigma G_{s} U^{\prime}\left(X_{s}\right) d B_{s}, \quad t \in\left(\tau_{n}, \tau_{n+1}\right] .
$$

Then processes $\left\{Z_{t}\right\}$ is a martingale. ${ }^{2}$ Therefore, from relation (4.39) we have

$$
\begin{aligned}
\frac{\partial E\left[U\left(X_{\tau_{n+1}}\right) \mid \mathcal{H}_{\tau_{n}}\right]}{\partial T_{n}} & =E\left[\left[r\left(X_{\tau_{n+1}}-G_{\tau_{n+1}}\right)+\alpha G_{\tau_{n+1}}\right] U^{\prime}\left(X_{\tau_{n+1}}\right)\right. \\
& \left.+\frac{1}{2} \sigma^{2} G_{\tau_{n+1}}^{2} U^{\prime \prime}\left(X_{\tau_{n+1}}\right) \mid \mathcal{H}_{\tau_{n}}\right]
\end{aligned}
$$

${ }^{1}$ For details consult Theorem 32 of [22]

${ }^{2}$ The proof is given in appendix 3 of [7] 
By replacing the factor $\frac{\partial E\left[U\left(X_{\tau_{n+1}}\right) \mid \mathcal{H}_{\tau_{n}}\right]}{\partial T_{n}}$ in relation (4.37) with the right hand side of relation (4.40), results in the following equation :

$$
\begin{aligned}
& \delta e^{-\frac{\delta}{\nu} T_{n}} Q_{n}^{-\gamma} \frac{1}{\gamma}\left[(1-a) W_{\tau_{n}}-b\right]^{\gamma}= \\
& \delta e^{-\delta T_{n}} E\left[U\left(X_{\tau_{n+1}}\right) \mid \mathcal{H}_{\tau_{n}}\right]-e^{-\delta T_{n}} E\left[\left[r\left(X_{\tau_{n+1}}-G_{\tau_{n+1}}\right)\right.\right. \\
& \left.\left.\quad+\alpha G_{\tau_{n+1}}\right] U^{\prime}\left(X_{\tau_{n+1}}\right)+\frac{1}{2} \sigma^{2} G_{\tau_{n+1}}^{2} U^{\prime \prime}\left(X_{\tau_{n+1}}\right) \mid \mathcal{H}_{\tau_{n}}\right] .
\end{aligned}
$$

By insertion of the withdrawal process $W_{\tau_{n}}$ as given by (4.15) and the investment process $V_{\tau_{n}}$ as given by (4.16) into the total wealth process $X_{\tau_{n}}$ of (4.9), then the total wealth process $X_{\tau_{n}}$ may be written as

$$
X_{\tau_{n+1}}=(1-\varepsilon)\left(1-F_{n}\right)\left(X_{\tau_{n}}-Y_{n}\right)\left[e^{r T_{n}}+\pi_{n}\left(\Gamma_{n+1}-e^{r T_{n}}\right)\right]+Y_{n+1}
$$

Since $G_{\tau_{n+1}}=V_{\tau_{n}} \Gamma_{n+1}$, by application of the investment process $V_{\tau_{n}}$ of (4.16), then we have

$$
G_{\tau_{n+1}}=(1-\varepsilon)\left(1-F_{n}\right)\left(X_{\tau_{n}}-Y_{n}\right) \pi_{n} \Gamma_{n+1} .
$$

Based on the optimal value function $U\left(X_{\tau_{n}}\right)$ of ( 4.14) and the total wealth process $X_{\tau_{n}}$ of $(4.42)$, then we have the following equations :

$$
\begin{aligned}
E\left[U\left(X_{\tau_{n+1}}\right) \mid \mathcal{H}_{\tau_{n}}\right] & =Q_{n+1}^{\nu} A_{n+1}^{-\nu}(1-\varepsilon)^{\gamma}\left(1-F_{n}\right)^{\gamma} \frac{1}{\gamma}\left(X_{\tau_{n}}-Y_{n}\right)^{\gamma} \\
& \times E\left(\left[e^{r T_{n}}+\pi_{n}\left(\Gamma_{n+1}-e^{r T_{n}}\right)\right]^{\gamma}\right), \\
E\left[U^{\prime}\left(X_{\tau_{n+1}}\right) \mid \mathcal{H}_{\tau_{n}}\right] & =Q_{n+1}^{\nu} A_{n+1}^{-\nu}(1-\varepsilon)^{-\nu}\left(1-F_{n}\right)^{-\nu}\left(X_{\tau_{n}}-Y_{n}\right)^{-\nu} \\
& \times E\left(\left[e^{r T_{n}}+\pi_{n}\left(\Gamma_{n+1}-e^{r T_{n}}\right)\right]^{-\nu}\right), \\
E\left[U^{\prime \prime}\left(X_{\tau_{n+1}}\right) \mid \mathcal{H}_{\tau_{n}}\right] & =Q_{n+1}^{\nu} A_{n+1}^{-\nu}(1-\varepsilon)^{-\nu-1}\left(1-F_{n}\right)^{-\nu-1}\left(X_{\tau_{n}}-Y_{n}\right)^{-\nu-1} \\
& \times(-\nu) E\left(\left[e^{r T_{n}}+\pi_{n}\left(\Gamma_{n+1}-e^{r T_{n}}\right)\right]^{-\nu-1}\right) .
\end{aligned}
$$


By taking the first derivative of $\Omega_{n}$ as given in (4.20) with respect to $\pi_{n}$ results in

$$
\left.E\left(\left[e^{r T_{n}}+\pi_{n}\left(\Gamma_{n+1}-e^{r T_{n}}\right)\right]^{-\nu}\left[\Gamma_{n+1}-e^{r T_{n}}\right)\right]\right)=0 .
$$

Then the following equations hold :

$$
\begin{aligned}
\Omega_{n} & =E\left(e^{r T_{n}}\left[e^{r T_{n}}+\pi_{n}\left(\Gamma_{n+1}-e^{r T_{n}}\right)\right]^{-\nu}\right), \\
& =E\left(\Gamma_{n+1}\left[e^{r T_{n}}+\pi_{n}\left(\Gamma_{n+1}-e^{r T_{n}}\right)\right]^{-\nu}\right), \\
& =E\left(\Gamma_{n+1}^{2}\left[e^{r T_{n}}+\pi_{n}\left(\Gamma_{n+1}-e^{r T_{n}}\right)\right]^{-\nu-1}\right) .
\end{aligned}
$$

By applying relations (4.15), (4.42), (4.43), (4.44), ( 4.45), ( 4.46), (4.47), (4.48), (4.49), in relation (4.41), and arranging all terms result in

$$
\begin{aligned}
& \left(X_{\tau_{n}}-Y_{n}\right)\left\{\delta e^{-\frac{\delta}{\nu} T_{n}} Q_{n}^{-\gamma} F_{n}^{\gamma}+Q_{n+1}^{\nu} A_{n+1}^{-\nu}\left(1-F_{n}\right)^{\gamma} R_{n}^{1 / \nu}[-\delta\right. \\
& \left.\left.\quad+\gamma r+\gamma(\alpha-r) \pi_{n}-\frac{1}{2} \sigma^{2} \gamma(1-\gamma) \pi_{n}^{2}\right]\right\} \\
& \quad+\gamma r(1-\varepsilon)^{-1} e^{-r T_{n}} Q_{n+1}^{\nu} A_{n+1}^{-\nu} R_{n}\left(1-F_{n}\right)^{\gamma-1} Y_{n+1}=0
\end{aligned}
$$

By substitution of $F_{n}$ as given by relation (4.18) into (4.50) results in

$$
\begin{aligned}
& \left(X_{\tau_{n}}-Y_{n}\right)\left\{\delta e^{-\frac{\delta}{\nu} T_{n}} \frac{\left[A_{n+1} Q_{n}(1-a)^{1 / \nu}\right]^{\gamma}}{\left[A_{n+1} Q_{n}(1-a)^{1 / \nu}+Q_{n+1} R_{n}^{1 / \nu}\right] \gamma}\right. \\
& +Q_{n+1}^{\nu} A_{n+1}^{-\nu} R_{n}^{1 / \nu} \frac{\left[Q_{n+1} R_{n}^{1 / \nu}\right]^{\gamma}}{\left[A_{n+1} Q_{n}(1-a)^{1 / \nu}+Q_{n+1} R_{n}^{1 / \nu}\right] \gamma}[-\delta+\gamma r \\
& \left.\left.+\gamma(\alpha-r) \pi_{n}-\frac{1}{2} \sigma^{2} \gamma(1-\gamma) \pi_{n}^{2}\right]\right\} \\
& +\frac{\gamma r Q_{n+1}^{\nu} R_{n} Y_{n+1}}{(1-\varepsilon) e^{r T_{n}} A_{n+1}^{\nu}} \frac{\left[Q_{n+1} R_{n}^{1 / \nu}\right]^{-\nu}}{\left[A_{n+1} Q_{n}(1-a)^{1 / \nu}+Q_{n+1} R_{n}^{1 / \nu}\right]^{-\nu}}=0 .
\end{aligned}
$$

Finally, by dividing all terms of ( 4.51 ) by

$$
\frac{A_{n+1}^{-\nu}}{\left[A_{n+1} Q_{n}(1-a)^{1 / \nu}+Q_{n+1} R_{n}^{1 / \nu}\right] \gamma},
$$


results in the following equation :

$$
g\left(T_{n}\right)+\left(X_{\tau_{n}}-Y_{n}\right) h\left(T_{n}\right)=0,
$$

with $\mathrm{g}$ and $\mathrm{h}$ are defined as the following :

$$
\begin{aligned}
g\left(T_{n}\right)= & \gamma r(1-\varepsilon)^{-1} e^{-r T_{n}} Y_{n+1}\left[A_{n+1} Q_{n}(1-a)^{1 / \nu}+Q_{n+1} R_{n}^{1 / \nu}\right], \\
h\left(T_{n}\right) & =\delta e^{-\frac{\delta}{\nu} T_{n}} A_{n+1}(1-a)^{\gamma / \nu}+Q_{n+1} R_{n}^{1 / \nu}\left[-\delta+\gamma r+\gamma(\alpha-r) \pi_{n}\right. \\
& \left.-\frac{1}{2} \sigma^{2} \gamma(1-\gamma) \pi_{n}^{2}\right] .
\end{aligned}
$$

Therefore, a complete proof of the following theorem has been given.

Theorem 4.2 Let the control problem faced by the investor be defined by the problem (4.10) subject to (4.11). Then the optimal transaction intervals $T_{n}, n=1,2,3, \ldots$, satisfy the equation

$$
g\left(T_{n}\right)+\left(X_{\tau_{n}}-Y_{n}\right) h\left(T_{n}\right)=0,
$$

with $g$ and $h$ are defined by

$$
\begin{aligned}
g\left(T_{n}\right)= & \gamma r(1-\varepsilon)^{-1} e^{-r T_{n}} Y_{n+1}\left[A_{n+1} Q_{n}(1-a)^{1 / \nu}+Q_{n+1} R_{n}^{1 / \nu}\right], \\
h\left(T_{n}\right) & =\delta e^{-\frac{\delta}{\nu} T_{n}} A_{n+1}(1-a)^{\gamma / \nu}+Q_{n+1} R_{n}^{1 / \nu}\left[-\delta+\gamma r+\gamma(\alpha-r) \pi_{n}\right. \\
& \left.-\frac{1}{2} \sigma^{2} \gamma(1-\gamma) \pi_{n}^{2}\right] .
\end{aligned}
$$


Theorem 4.3 Suppose that $\alpha, a, \delta, \varepsilon, \gamma, r, \sigma$ satisfying the following conditions :

$$
\begin{aligned}
& \text { 1. } \alpha-r \geq \frac{1}{2} \sigma^{2}(1-\gamma) \\
& \text { 2. } \max (\gamma \alpha, \gamma r)<\delta<\min \left(\frac{\gamma r(1-a)}{(1-\varepsilon)^{\gamma / \nu}}, r-\gamma r\right)
\end{aligned}
$$

If $F_{n}$ as defined by (4.18) has a property such that $F_{n} \geq Q_{n}\left(1-R_{n}^{1 / \nu}\right)$, then the equation

$$
g\left(T_{n}\right)+\left(X_{\tau_{n}}-Y_{n}\right) h\left(T_{n}\right)=0
$$

as defined in Theorem 4.2 has a solution.

Proof : Let $H\left(T_{n}\right)=g\left(T_{n}\right)+\left(X_{\tau_{n}}-Y_{n}\right) h\left(T_{n}\right)$, with $g$ and $h$ are given by relations ( 4.56$)$ and (4.57) respectively. It will be shown that $H\left(T_{n}\right)=0$ has a solution. Notice that, $H$ is a continuous function in $T_{n}$. Then it will be shown that $H>0$ as $T_{n} \rightarrow 0^{+}$and $H<0$ as $T_{n}$ large. By using $g$ and $h$ as given by (4.56) and (4.57), then

$$
\begin{aligned}
\lim _{T_{n} \rightarrow 0^{+}} H\left(T_{n}\right) & =\lim _{T_{n} \rightarrow 0^{+}} \frac{\gamma r Y_{n+1}\left[A_{n+1} Q_{n}(1-a)^{1 / \nu}+Q_{n+1} R_{n}^{1 / \nu}\right]}{(1-\varepsilon) e^{r T_{n}}} \\
& +\lim _{T_{n} \rightarrow 0^{+}} Q_{n+1}\left(X_{\tau_{n}}-Y_{n}\right)\left\{\delta e^{-\delta / \nu T_{n}} \frac{A_{n+1}}{Q_{n+1}}(1-a)^{\gamma / \nu}\right. \\
& \left.+R_{n}^{1 / \nu}\left[-\delta+\gamma r+\gamma(\alpha-r) \pi_{n}-1 / 2 \sigma^{2} \gamma(1-\gamma) \pi_{n}^{2}\right]\right\} .
\end{aligned}
$$

By Remark 4.3, $\frac{A_{n+1}}{Q_{n+1}} \geq \frac{R_{n}^{1 / \nu}\left(1-R_{n}^{1 / \nu}\right)}{(1-a)^{1 / \nu}}$ for $F_{n} \geq Q_{n}\left(1-R_{n}^{1 / \nu}\right)$. This and relation (4.19) imply that

$$
\begin{aligned}
\lim _{T_{n} \rightarrow 0^{+}} H\left(T_{n}\right) & \geq \lim _{T_{n} \rightarrow 0^{+}} \frac{\gamma r Y_{n+1}\left[A_{n+1} Q_{n}(1-a)^{1 / \nu}+Q_{n+1} R_{n}^{1 / \nu}\right]}{(1-\varepsilon) e^{r T_{n}}} \\
& +\lim _{T_{n} \rightarrow 0^{+}} Q_{n+1}\left(X_{\tau_{n}}-b-a W_{\tau_{n}}-(1-\varepsilon)^{-1} e^{-r T_{n}} Y_{n+1}\right) \\
& \times\left\{\delta e^{-\delta / \nu T_{n}} R_{n}^{1 / \nu}\left(1-R_{n}^{1 / \nu}\right)(1-a)^{-1}\right. \\
& \left.+R_{n}^{1 / \nu}\left[-\delta+\gamma r+\gamma(\alpha-r) \pi_{n}-1 / 2 \sigma^{2} \gamma(1-\gamma) \pi_{n}^{2}\right]\right\} .
\end{aligned}
$$


From the assumption of the theorem, we have that $(\alpha-r) \geq \frac{1}{2} \sigma^{2}(1-\gamma)$. Furthermore, from its definition in relation (4.20), $\pi_{n} \in[0,1]$. These imply that

$$
-\delta+\gamma r+\gamma(\alpha-r) \pi_{n}-1 / 2 \sigma^{2} \gamma(1-\gamma) \pi_{n}^{2} \geq-\delta+\gamma r .
$$

Therefore,

$$
\begin{aligned}
\lim _{T_{n} \rightarrow 0^{+}} H\left(T_{n}\right) & \geq \gamma r(1-\varepsilon)^{-1+\gamma / \nu} Y_{n+1}(1-a)^{1 / \nu} Q_{n+1} \\
& +(1-\varepsilon)^{\gamma / \nu} Q_{n+1}\left(X_{\tau_{n}}-b-a W_{\tau_{n}}-(1-\varepsilon)^{-1} Y_{n+1}\right) \\
& \times\left\{\delta\left[1-(1-\varepsilon)^{\gamma / \nu}\right](1-a)^{-1}+(-\delta+\gamma r)\right\} .
\end{aligned}
$$

By assumption of the theorem we have that $\delta<\frac{(1-a) \gamma r}{(1-\varepsilon)^{\gamma / \nu}}$. This implies that

$$
\delta\left[1-(1-\varepsilon)^{\gamma / \nu}\right](1-a)^{-1}+(-\delta+\gamma r)>0 .
$$

Therefore,

$$
\lim _{T_{n} \rightarrow 0^{+}} H\left(T_{n}\right)>\gamma r(1-\varepsilon)^{-1+\gamma / \nu} Y_{n+1}(1-a)^{1 / \nu} Q_{n+1}
$$

Since $a, \varepsilon, \gamma, Q_{n+1}, \quad r, Y_{n+1}$ are all nonnegatives, then we have that $\lim _{T_{n} \rightarrow 0^{+}} H\left(T_{n}\right)>0$.

On the other hand, we have that

$$
\begin{aligned}
H\left(T_{n}\right) & =\frac{\gamma r}{(1-\varepsilon) e^{r T_{n}}} Y_{n+1}\left[A_{n+1} Q_{n}(1-a)^{1 / \nu}+Q_{n+1} R_{n}^{1 / \nu}\right] \\
& +\left(X_{\tau_{n}}-Y_{n}\right)\left\{\delta e^{-\frac{\delta}{\nu} T_{n}} A_{n+1}(1-a)^{\gamma / \nu}+Q_{n+1} R_{n}^{1 / \nu}\right. \\
& \left.\times\left[-\delta+\gamma r+\gamma(\alpha-r) \pi_{n}-\frac{1}{2} \sigma^{2} \gamma(1-\gamma) \pi_{n}^{2}\right]\right\}
\end{aligned}
$$

Since we have that $A_{n+1}, \quad Q_{n+1}, \quad$ and $\quad Q_{n}$ are less than or equals to unity, and that $R_{n}^{1 / \nu} \leq(1-\varepsilon)^{\gamma / \nu}$, then 


$$
\begin{aligned}
H\left(T_{n}\right) & \leq \frac{\gamma r}{(1-\varepsilon) e^{r T_{n}}} Y_{n+1}\left[(1-a)^{1 / \nu}+(1-\varepsilon)^{\gamma / \nu}\right] \\
& +\left(X_{\tau_{n}}-Y_{n}\right)\left\{\delta e^{-\frac{\delta}{\nu} T_{n}}(1-a)^{\gamma / \nu}+Q_{n+1} R_{n}^{1 / \nu}\right. \\
& \times[-\delta+\max (\gamma \alpha, \gamma r)]\} .
\end{aligned}
$$

By rearranging the terms, then we have

$$
\begin{aligned}
H\left(T_{n}\right) & \leq e^{-\frac{\delta}{\nu} T_{n}}\left(X_{\tau_{n}}-Y_{n}\right)\left\{\frac{\gamma r e^{\frac{\delta}{\nu} T_{n}} Y_{n+1}\left[(1-a)^{1 / \nu}+(1-\varepsilon)^{\gamma / \nu}\right]}{(1-\varepsilon) e^{r T_{n}}\left(X_{\tau_{n}}-Y_{n}\right)}\right. \\
& \left.+\delta+Q_{n+1} R_{n}^{1 / \nu} e^{\delta / \nu T_{n}}[-\delta+\max (\gamma \alpha, \gamma r)]\right\}
\end{aligned}
$$

Since by assumptions of the theorem that $\delta>\max (\gamma \alpha, \gamma r)$, and $r>\delta / \nu$, then $H\left(T_{n}\right)<0$ for large $T_{n}$.

It has been shown that $H\left(T_{n}\right)>0$ as $T_{n} \rightarrow 0^{+}$and $H\left(T_{n}\right)<0$ as $T_{n}$ large. Since $H\left(T_{n}\right)$ is continuous in $T_{n}$, then there exists $\hat{T}_{n}$ such that $H\left(\hat{T}_{n}\right)=0$. Therefore, the proof of Theorem 4.3 has been completed

Theorem 4.4 Suppose that $\alpha, a, \delta, \varepsilon, \gamma, r, \sigma$ satisfying the following conditions :

1. $\alpha-r \geq \frac{1}{2} \sigma^{2}(1-\gamma)$

2. $\max (\gamma \alpha, \gamma r)<\delta<\min \left(\frac{\gamma r(1-a)}{(1-\varepsilon)^{\gamma / \nu}}, r-\gamma r\right)$

If $F_{n}$ as defined by (4.18) has a property such that $F_{n} \geq Q_{n}\left(1-R_{n}^{1 / \nu}\right)$, then an optimal policy $(T, W, V, C)$ exists.

Proof : Consider $H\left(T_{n}\right)=g\left(T_{n}\right) Y_{n+1}+\left(X_{\tau_{n}}-Y_{n}\right) h\left(T_{n}\right)$. By Remark 4.3, $\frac{A_{n+1}}{Q_{n+1}} \geq \frac{R_{n}^{1 / \nu}\left(1-R_{n}^{1 / \nu}\right)}{(1-a)^{1 / \nu}}$ for $F_{n} \geq Q_{n}\left(1-R_{n}^{1 / \nu}\right)$. Then by Theorem 4.3, there 
exists a scalar $\hat{T}_{n}>0$ such that $H\left(\hat{T}_{n}\right)=0$. The arguments in the proof of Theorem 4.3 show that one of the solutions, say $\bar{T}_{n}$, corresponds to the maximum of (4.9).

Now consider the following withdrawal and investment policies and function $f$, for $n=1,2,3, \ldots$

$$
\begin{aligned}
f\left(X_{\tau_{n}}\right) & =Q_{n}^{\nu} A_{n}^{-\nu} \frac{1}{\gamma}\left(X_{\tau_{n}}-Y_{n}\right)^{\gamma}, \\
W_{\tau_{n}} & =\frac{F_{n}}{1-a}\left(X_{\tau_{n}}-Y_{n}\right)+\frac{b}{1-a}, \\
V_{\tau_{n}} & =(1-\varepsilon)\left(1-F_{n}\right)\left(X_{\tau_{n}}-Y_{n}\right) \pi_{n}, \\
T_{n} & =\hat{T}_{n} .
\end{aligned}
$$

By Lemma 4.3 then $f\left(X_{\tau_{n}}\right)$ satisfies $(4.10)$, and $\left(T_{n}, W_{\tau_{n}}, V_{\tau_{n}}\right)$ achieves the maximum in $(4.12)$ for all $n=1,2,3, \ldots$. It will be shown that $\lim _{n \rightarrow \infty} E\left[e^{-\delta \tau_{n+1}} f\left(X_{\tau_{n+1}}\right)\right]=0$.

Notice that, by using relation ( 4.58$) E\left[e^{-\delta \tau_{n+1}} f\left(X_{\tau_{n+1}}\right)\right]$ may be expressed as

$$
E\left[e^{-\delta \tau_{n+1}} f\left(X_{\tau_{n+1}}\right)\right]=Q_{n+1}^{\nu} A_{n+1}^{-\nu} e^{-\delta \tau_{n+1}} \frac{1}{\gamma} E\left[\left(X_{\tau_{n+1}}-Y_{n+1}\right)^{\gamma} \mid \mathcal{H}_{\tau_{n}}\right],
$$

with the total wealth process $X_{\tau_{n+1}}$ is as in (4.11). By applying the withdrawal process $W_{\tau_{n}}$ and the investment process $V_{\tau_{n}}$ as given by relations (4.59) and (4.60) respectively in (4.11), then the expectation factor on the right hand side of ( 4.62) may be written as

$$
E\left[\left(X_{\tau_{n+1}}-Y_{n+1}\right)^{\gamma} \mid \mathcal{H}_{\tau_{n}}\right]=(1-\varepsilon)^{\gamma} \Omega_{n}\left(1-F_{n}\right)^{\gamma}\left[\left(X_{\tau_{n}}-Y_{n}\right)^{\gamma} \mid \mathcal{H}_{\tau_{n-1}}\right]
$$

Also notice that $\tau_{n+1}=T_{n}+\tau_{n}$. By letting $D \equiv \frac{1}{\gamma} Q_{n+1}^{\nu} A_{n+1}^{-\nu}$, then 
relation ( 4.62) may be written as

$$
E\left[e^{-\delta \tau_{n+1}} f\left(X_{\tau_{n+1}}\right)\right]=D R_{n}\left(1-F_{n}\right)^{\gamma} e^{-\delta \tau_{n}}\left[\left(X_{\tau_{n}}-Y_{n}\right)^{\gamma} \mid \mathcal{H}_{\tau_{n-1}}\right]
$$

By induction it follows that

$$
\begin{aligned}
E & {\left[e^{-\delta \tau_{n+1}} f\left(X_{\tau_{n+1}}\right)\right] } \\
& =D R_{n}\left(1-F_{n}\right)^{\gamma} e^{-\delta \tau_{n}}\left[\left(X_{\tau_{n}}-Y_{n}\right)^{\gamma} \mid \mathcal{H}_{\tau_{n-1}}\right] \\
& =D R_{n}\left(1-F_{n}\right)^{\gamma} R_{n-1}\left(1-F_{n-1}\right)^{\gamma} e^{-\delta \tau_{n-1}}\left[\left(X_{\tau_{n-1}}-Y_{n-1}\right)^{\gamma} \mid \mathcal{H}_{\tau_{n-2}}\right] \\
& =D R_{n}\left(1-F_{n}\right)^{\gamma} \ldots R_{1}\left(1-F_{1}\right)^{\gamma} e^{-\delta \tau_{1}}\left[\left(X_{\tau_{1}}-Y_{1}\right)^{\gamma}\right] \\
& \leq D X_{0}^{\gamma} \prod_{k=1}^{n}\left[R_{k}\left(1-F_{k}\right)^{\gamma}\right] \leq D X_{0}^{\gamma} \prod_{k=1}^{n}\left[(1-\varepsilon)^{\gamma / \nu}\right]
\end{aligned}
$$

as $R_{n} \leq(1-\varepsilon)^{\gamma / \nu}$ and $0<1-F_{n} \leq 1$ for any feasible policy. Since $D$ is bounded, then

$$
\lim _{n \rightarrow \infty} E\left[e^{-\delta \tau_{n}} f\left(X_{\tau_{n}}\right)\right]=0 .
$$

Therefore, condition (ii) of Lemma 4.3 is satisfied. Then by Lemma 4.3 , the proof of Theorem 4.4 has been completed 


\section{Chapter 5}

\section{Portfolio Selection with}

\section{Nonlinear Transaction Costs}

\subsection{Introduction}

Chapter 5 is concerned with consumption and investment strategy for an investor who seeks to maximize the expected utility of consumption. The investor has available a riskless asset paying fixed interest rate and a risky asset with logarithmic Brownian motion price fluctuations. The objective is to maximize the expected discounted utility of consumption. The investor observes his/her current wealth and makes transaction at stopping times $\tau_{1}, \tau_{2}, \tau_{3}, \ldots$. The decision to transact can be made at any time based on all current information. For every transaction, the investor is charged a fraction of the current wealth as a management fee plus transaction costs which is a nonlinear function of the amount of wealth transacted. 
The problem faced by the investor, as formulated in Chapter 2, is in a discrete-continuous time optimal control problem form. The optimal control problem for the investor is to choose optimal policy $(T, W, V, C)$ in a set of feasible policies $\mathcal{U}$, such that the value function is maximized. The main task of Chapter 3 is to find an optimal solution to that optimal control problem. Analysis in Chapter 5 is similar to those in Chapter 3 and Chapter 4. As in Chapter 3 and Chapter 4, we will solve the problem in two steps. In the first step, a deterministic continuous-time optimal control problem for consumption is solved, given $(T, W, V)$. In the second step, equipped with the optimal value function from the first step, a stochastic discrete-time optimal control problem is solved. The existence of an optimal consumption and investment selection is given.

The rest of Chapter 5 is organized as follows. Section 5.2 states the problem which is formulated in Chapter 2. In Section 5.3, we show that it is not optimal for the investor to withdraw more money than the amount needed for consumption. For a given $(T, W, V)$, the optimal consumption for the investor is solved. Section 5.4 is concerned with the derivation of optimal money withdrawals process and investment strategy for the risky security. This is done by deriving the optimal value function for fixed interval $T_{n}$ for all $\mathrm{n}$. This is one of the main features of Chapter 5 as well as this thesis. In Section 5.5, with optimal $(W, V, C)$ in hand, an equation satisfied by the transaction intervals is derived. Each optimal transaction interval infact satisfies a nonlinear equation which is not independent of total wealth at the beginning of that interval. A solution to that equation exists and is optimal. 


\subsection{Statement of the Problem}

The model under investigation in Chapter 5 is as formulated in Chapter 2, with nonlinear transaction costs. The transaction costs function $\Phi$ is nonlinear, non-decreasing, concave and sufficiently smooth function of its argument. The money holding at any given time $\mathrm{t}$ is defined by

$$
M_{t}=\sum_{\left\{n: \tau_{n} \leq t\right\}}\left[W_{\tau_{n}}-\Phi\left(W_{\tau_{n}}\right)\right]-\int_{0}^{t} C_{s} d s
$$

Definition 5.1 Let $\mathcal{U}$ be the set of all budget feasible policies as defined in Chapter 2. The optimal control problem for the investor is

$$
U\left(X_{0}\right) \equiv \max _{(T, W, V, C) \in \mathcal{U}} E\left[\int_{0}^{\infty} e^{-\delta t} u\left(C_{t}\right) d t\right]
$$

subject to, for $n=1,2,3, \ldots$

$$
X_{\tau_{n+1}}=(1-\varepsilon)\left[X_{\tau_{n}}-W_{\tau_{n}}\right] e^{r T_{n}}+V_{\tau_{n}}\left[\Gamma_{n+1}-e^{r T_{n}}\right]
$$

and $M_{t} \geq 0$, and $\quad X_{\tau_{n+1}} \geq 0$.

\subsection{Consumption Strategy}

Section 5.3 is concerned with the derivation of consumption strategy for the investor who has the optimal control problem as given by relations (5.2)(5.3). As it was the case for bullet transaction costs problem in Section 3.3 and the linear transaction costs problem in Section 4.3, it is not optimal for the investor to take out more money than needed for consumption during an interval. This is proved in Lemma 5.1 
Lemma 5.1 Let the value function $U$ be defined as in (5.2), and let the transaction cost function $\Phi$ be a nonlinear, nondecreasing, concave and smooth function of the amount of wealth withdrawn. Then the optimal policy $(T, W, V, C)$ must satisfy for all $n=1,2,3, \ldots$

$$
\int_{\tau_{n}}^{\tau_{n+1}} C_{t} d t=W_{\tau_{n}}-\Phi\left(W_{\tau_{n}}\right) .
$$

Proof : The proof is similar to those of Lemma 3.1 and Lemma 4.1

Suppose that there exists an interval $T_{j}=\tau_{j+1}-\tau_{j}$ such that

$$
d=W_{\tau_{j}}-\Phi\left(W_{\tau_{j}}\right)-\int_{\tau_{j}}^{\tau_{j+1}} C_{t} d t>0
$$

Because there exists a riskless security with a positive interest rate, then the investor will be better off if he/she invests the amount $d$ in the riskless security during the interval $T_{j}$, and consumed the interest income $d\left(e^{r T_{j}}-1\right)$ in the next interval. In other words, the optimal policy $(T, W, V, C)$ is dominated by a feasible policy $(T, \bar{W}, V, \bar{C})$ which is defined as the following : choose $\bar{W}_{\tau_{j}}$ such that

$$
\bar{W}_{\tau_{j}}=\Phi\left(\bar{W}_{\tau_{j}}\right)-\int_{\tau_{j}}^{\tau_{j+1}} C_{t} d t
$$

and define $\bar{W}_{\tau_{j+1}}$ and $\bar{C}_{t}$ as the following :

$$
\begin{gathered}
\bar{W}_{\tau_{j+1}}=W_{\tau_{j+1}}+d e^{r T_{j}}>W_{\tau_{j+1}}, \\
\bar{C}_{t}=C_{t}+\frac{1}{T_{j+1}} d\left(e^{r T_{j}}-1\right)>C_{t}, \quad t \in\left[\tau_{j+1}, \tau_{j+2}\right) \\
\bar{C}_{t}=C_{t}, \quad \bar{W}_{\tau_{j}}=W_{\tau_{j}}, \quad \text { otherwise. }
\end{gathered}
$$

But $(T, W, V, C)$ is optimal. Hence contradiction. Therefore, for all $n$,

$$
\int_{\tau_{n}}^{\tau_{n+1}} C_{t} d t \geq W_{\tau_{n}}-\Phi\left(W_{\tau_{n}}\right)
$$


On the other hand, since the expenditure must be financed from the stock of money, for all $n$,

$$
\sum_{i}^{n} \int_{\tau_{i}}^{\tau_{i+1}} C_{t} d t \geq \sum_{i}^{n} W_{\tau_{i}}-\Phi\left(W_{\tau_{i}}\right)
$$

Therefore, for all $n$, an optimal policy $(T, W, V, C)$ must satisfy

$$
\int_{\tau_{n}}^{\tau_{n+1}} C_{t} d t=W_{\tau_{n}}-\Phi\left(W_{\tau_{n}}\right)
$$

Corollary 5.1 By the definition of money holding $M_{t}$, then

$$
M_{\tau_{n}}=W_{\tau_{n}}-\Phi\left(W_{\tau_{n}}\right), \quad n=1,2,3, \ldots
$$

Therefore, the optimal control problem (5.2)-( 5.3) is equivalent to the optimal control problem :

$$
U\left(X_{0}\right)=\max _{(T, W, V, C) \in \mathcal{U}} E\left[\int_{0}^{\infty} e^{-\delta t} u\left(C_{t}\right) d t\right]
$$

subject to, for $n=1,2,3, \ldots$

$$
\begin{gathered}
\int_{\tau_{n}}^{\tau_{n+1}} C_{t} d t=W_{\tau_{n}}-\Phi\left(W_{\tau_{n}}\right), \\
X_{\tau_{n+1}}=(1-\varepsilon)\left[X_{\tau_{n}}-W_{\tau_{n}}\right] e^{r T_{n}}+V_{\tau_{n}}\left[\Gamma_{n+1}-e^{r T_{n}}\right] \geq 0 .
\end{gathered}
$$

As it is the case in the previous Chapters, the value function $U$ will be re-written as

$$
U\left(X_{0}\right)=E\left[\sum_{n=1}^{\infty} e^{-\delta \tau_{n}} \int_{\tau_{n}}^{\tau_{n+1}} e^{-\delta\left(t-\tau_{n}\right)} u\left(C_{t}\right) d t\right]
$$

Then the optimal control problem ( 5.5)-( 5.7) will be solved in two steps. In the first step, a deterministic continuous-time optimal control problem is 
solved for any given $(T, W, V)$. In the second step, equipped with the optimal value function of the problem in the first step, a stochastic discrete-time optimal control problem is solved.

The deterministic continuous-time optimal control problem which will be solved in the first step is : maximize the objective function

$$
J(t, Z)=\int_{0}^{t} e^{-\delta s} u\left(C_{s}\right) d s
$$

over $\left\{C_{s}: 0 \leq s \leq t\right\}$, subject to :

$$
\int_{0}^{t} C_{s} d s \leq Z
$$

where

$$
u\left(C_{t}\right)=\frac{1}{\gamma} C_{t}^{\gamma}, \quad 0<\gamma<1 .
$$

The following result is similar to those of Section 3.3 and Section 4.3.

Lemma 5.2 The optimal value function $J$ for the above optimal control problem is given by

$$
J(t, Z)=\left(\frac{1-\gamma}{\delta}\right)^{1-\gamma}\left[1-\exp \left(-\frac{\delta}{1-\gamma} t\right)\right]^{1-\gamma} \frac{1}{\gamma} Z^{\gamma} .
$$

By Corollary 5.1 then

$$
J\left(T_{n}, M_{\tau_{n}}\right)=\left[1-\exp \left(-\frac{\delta}{1-\gamma} T_{n}\right)\right]^{1-\gamma} \frac{1}{\gamma}\left(W_{\tau_{n}}-\Phi\left(W_{\tau_{n}}\right)\right)^{\gamma},
$$

with the term $[(1-\gamma) / \delta]^{1-\gamma}$ has been left out.

Let $Q_{n}=1-\exp \left(-\frac{\delta}{\nu} T_{n}\right)$, with $\nu=1-\gamma$. Then the modified control problem is given by

$$
U\left(X_{0}\right)=\max _{\{T \in \mathcal{T}, W \in \mathcal{W}, V \in \mathcal{V}\}} E\left[\sum_{n=1}^{\infty} e^{-\delta \tau_{n}} Q_{n}^{\nu} \frac{1}{\gamma}\left[W_{\tau_{n}}-\Phi\left(W_{\tau_{n}}\right)\right]^{\gamma}\right],
$$


subject to, for $n=1,2,3, \ldots$,

$$
X_{\tau_{n+1}}=(1-\varepsilon)\left[X_{\tau_{n}}-W_{\tau_{n}}\right] e^{r T_{n}}+V_{\tau_{n}}\left[\Gamma_{n+1}-e^{r T_{n}}\right] \geq 0 .
$$

The application of the principle of optimality of Bellman on the above control problem implies that, for $n=1,2,3, \ldots$

$$
U\left(X_{\tau_{n}}\right)=\max _{\left\{T_{n}, W_{\tau_{n}}, V_{\tau_{n}}\right\}}\left\{Q_{n}^{\nu} \frac{1}{\gamma}\left[W_{\tau_{n}}-\Phi\left(W_{\tau_{n}}\right)\right]^{\gamma}+e^{-\delta T_{n}} E\left[U\left(X_{\tau_{n+1}}\right) \mid \mathcal{H}_{\tau_{n}}\right]\right\},
$$

subject to, for $n=1,2,3, \ldots$,

$$
X_{\tau_{n+1}}=(1-\varepsilon)\left[X_{\tau_{n}}-W_{\tau_{n}}\right] e^{r T_{n}}+V_{\tau_{n}}\left[\Gamma_{n+1}-e^{r T_{n}}\right] \geq 0 .
$$

Lemma 5.3 Let $\bar{Q}(n)=\left[1-\exp \left(-\frac{\delta}{\nu} T_{n}\right)\right]^{\nu}$. Suppose that $f$ is a realvalued function on $[0, \infty)$ satisfying the two conditions :

(i) For all $n=1,2,3, \ldots$,

$$
\begin{aligned}
f\left(X_{\tau_{n}}\right) & =\max _{\left(T_{n}, W_{\tau_{n}}, V_{\tau_{n}}\right)}\left\{\bar{Q}(n) \frac{1}{\gamma}\left(W_{\tau_{n}}-\Phi\left(W_{\tau_{n}}\right)\right)^{\gamma}\right. \\
& \left.+E\left[e^{-\delta T_{n}} f\left(X_{\tau_{n+1}}\right)\right] \mid \mathcal{H}_{\tau_{n}}\right\} .
\end{aligned}
$$

(ii) For any feasible policy,

$$
\lim _{n \rightarrow \infty} E\left[e^{-\delta \tau_{n}} f\left(X_{\tau_{n}}\right)\right]=0 .
$$

If $\left(T^{*}, W^{*}, V^{*}\right)$ achieves the maximum in (5.12) for all $n$ then $f$ is the value function for the control problem (5.8), and $\left(T^{*}, W^{*}, V^{*}\right)$ is an optimal policy.

Proof : Let $n=1$, that is $\tau_{1}=0$. Then

$$
\begin{aligned}
f\left(X_{0}\right) & =\max _{\left(T_{1}, W_{0}, V_{0}\right)}\left\{\bar{Q}(1) \frac{1}{\gamma}\left(W_{0}-\Phi\left(W_{0}\right)\right)^{\gamma}+E\left[e^{-\delta T_{1}} f\left(X_{\tau_{2}}\right)\right]\right\} \\
& \geq \bar{Q}(1) \frac{1}{\gamma}\left(W_{0}-\Phi\left(W_{0}\right)\right)^{\gamma}+E\left[e^{-\delta T_{1}} f\left(X_{\tau_{2}}\right)\right],
\end{aligned}
$$


for any feasible $\left(T_{1}, W_{0}, V_{0}\right)$. By induction, for any $(T, W, V) \in \mathcal{T} \times \mathcal{W} \times \mathcal{V}$ then

$$
f\left(X_{0}\right) \geq E\left[\sum_{n=1}^{\infty} e^{-\delta \tau_{n}} \bar{Q}_{n} \frac{1}{\gamma}\left[W_{\tau_{n}}-\Phi\left(W_{\tau_{n}}\right)\right]^{\gamma}\right] .
$$

This holds for an arbitrary feasible policy (T,W,V). Hence, $f\left(X_{0}\right) \geq U\left(X_{0}\right)$. On the other hand, $U\left(X_{0}\right) \geq f\left(X_{0}\right)$. Hence, $f\left(X_{0}\right)=U\left(X_{0}\right)$, and consequently $\left(T^{*}, W^{*}, V^{*}\right)$ is optimal 


\subsection{Investment Strategies}

Section 5.4 is mainly concerned with the derivation of optimal value function for the problem as defined by relations (5.10) subject to the total wealth process $X_{\tau_{n}}$ as given by relation (5.11). A closed form of the value function, as well as the withdrawal and investment strategies are derived for fixed transaction intervals $T_{n}$. The analysis in Section 5.4 is similar to that of Section 3.4 and Section 4.4 .

Theorem 5.1 Let $T_{n}$ be fixed for $n=1,2,3, \ldots$. Then the optimal value function and unique solution to the problem (5.10) is given by

$$
U\left(X_{\tau_{n}}\right)=Q_{n}^{\nu} A_{n}^{-\nu} \frac{1}{\gamma}\left(X_{\tau_{n}}-Y_{n}\right)^{\gamma},
$$

and the withdrawal and investment strategies are given by

$$
\begin{aligned}
W_{\tau_{n}} & =F_{n}\left(X_{\tau_{n}}-Y_{n}\right)+\Phi\left(W_{\tau_{n}}\right), \\
V_{\tau_{n}} & =(1-\varepsilon)\left(1-F_{n}\right)\left(X_{\tau_{n}}-Y_{n}\right) \pi_{n},
\end{aligned}
$$

where $A_{n}, F_{n}$ and $Y_{n}$ are given by

$$
\begin{aligned}
A_{n} & =\frac{A_{n+1} Q_{n}\left[A_{n+1} Q_{n}\left(1-\Phi^{\prime}\left(W_{\tau_{n}}\right)\right)^{1 / \nu}+Q_{n+1} R_{n}^{1 / \nu}\right]^{\gamma / \nu}}{\left[A_{n+1} Q_{n}\left(1-\Phi^{\prime}\left(W_{\tau_{n}}\right)\right)^{\gamma / \nu}+Q_{n+1} R_{n}^{1 / \nu}\right]^{1 / \nu}} \\
F_{n} & =\frac{A_{n+1} Q_{n}\left(1-\Phi^{\prime}\left(W_{\tau_{n}}\right)\right)^{1 / \nu}}{A_{n+1} Q_{n}\left(1-\Phi^{\prime}\left(W_{\tau_{n}}\right)\right)^{1 / \nu}+Q_{n+1} R_{n}^{1 / \nu}} \\
Y_{n} & =\Phi\left(W_{\tau_{n}}\right)+(1-\varepsilon)^{-1} e^{-r T_{n}} Y_{n+1}
\end{aligned}
$$

with $R_{n}$ is given by $R_{n}=(1-\varepsilon)^{\gamma} e^{-\delta T_{n}} \Omega_{n}$, and where $\Omega_{n}$ and $\pi_{n}$ are defined by the optimization problem :

$$
\begin{aligned}
\Omega_{n} & \equiv E\left(\left[e^{r T_{n}}+\pi_{n}\left(\Gamma_{n+1}-e^{r T_{n}}\right)\right]^{\gamma}\right) \\
& \equiv \sup _{\{0 \leq \pi \leq 1\}} E\left(\left[e^{r T_{n}}+\pi\left(\Gamma_{n+1}-e^{r T_{n}}\right)\right]^{\gamma}\right) .
\end{aligned}
$$


Proof : The idea of the proof is similar to that of Theorem 3.1 and Theorem 4.1. Let denote the right-hand side of relation ( 5.10) by $S\left(X_{\tau_{n}}\right)$ upon inserting relation ( 5.14) for $U\left(X_{\tau_{n}}\right)$. Therefore, $S\left(X_{\tau_{n}}\right)$ is given by

$$
\begin{aligned}
S\left(X_{\tau_{n}}\right) & =\max _{\left\{W_{\tau_{n}}, V_{\tau_{n}}\right\}}\left\{Q_{n}^{\nu} \frac{1}{\gamma}\left(W_{\tau_{n}}-\Phi\left(W_{\tau_{n}}\right)\right)^{\gamma}\right. \\
& \left.+Q_{n+1}^{\nu} A_{n+1}^{-\nu} e^{-\delta T_{n}} E\left[\frac{1}{\gamma}\left(X_{\tau_{n+1}}-Y_{n+1}\right)^{\gamma} \mid \mathcal{H}_{\tau_{n}}\right]\right\} .
\end{aligned}
$$

Let define $Y_{n}$ by the following recurrence relationship :

$$
Y_{n}=\Phi\left(W_{\tau_{n}}\right)+(1-\varepsilon)^{-1} e^{-r T_{n}} Y_{n+1}
$$

Then the total wealth process $X_{\tau_{n+1}}$ as defined by relation ( 5.11) may be written as

$$
\begin{aligned}
X_{\tau_{n+1}} & =(1-\varepsilon)\left[X_{\tau_{n}}-Y_{n}-\left(W_{\tau_{n}}-\Phi\left(W_{\tau_{n}}\right)\right)\right] e^{r T_{n}} \\
& +V_{\tau_{n}}\left(\Gamma_{n+1}-e^{r T_{n}}\right)+Y_{n+1} .
\end{aligned}
$$

By insertion of the total wealth $X_{\tau_{n+1}}$ as given by relation ( 5.22) into $S\left(X_{\tau_{n}}\right)$ yields

$$
\begin{aligned}
S\left(X_{\tau_{n}}\right) & =\max _{\left\{W_{\tau_{n}}, V_{\tau_{n}}\right\}}\left\{Q_{n}^{\nu} \frac{1}{\gamma}\left(W_{\tau_{n}}-\Phi\left(W_{\tau_{n}}\right)\right)^{\gamma}+A_{n+1}^{-\nu} Q_{n+1}^{\nu} e^{-\delta T_{n}}\right. \\
& \times E\left[\frac { 1 } { \gamma } \left[(1-\varepsilon)\left[X_{\tau_{n}}-Y_{n}-\left(W_{\tau_{n}}-\Phi\left(W_{\tau_{n}}\right)\right)\right] e^{r T_{n}}\right.\right. \\
& \left.\left.\left.+V_{\tau_{n}}\left(\Gamma_{n+1}-e^{r T_{n}}\right)\right]^{\gamma}\right]\right\},
\end{aligned}
$$

subject to the following constraints :

$$
\begin{aligned}
W_{\tau_{n}}-\Phi\left(W_{\tau_{n}}\right) & \geq 0 \\
P\left\{X_{\tau_{n+1}} \geq Y_{n+1}\right\} & =1 \\
V_{\tau_{n}} & \geq 0 .
\end{aligned}
$$


Let $I_{n}$ be defined as

$$
I_{n}=\frac{V_{\tau_{n}}}{(1-\varepsilon)\left[X_{\tau_{n}}-Y_{n}-\left(W_{\tau_{n}}-\Phi\left(W_{\tau_{n}}\right)\right)\right]} .
$$

Then by re-arrangement, for $X_{\tau_{n}}-Y_{T_{n}}-\left(W_{\tau_{n}}-\Phi\left(W_{\tau_{n}}\right)\right)>0$, the total wealth process $X_{\tau_{n+1}}$ as given by relation (5.22) may also be written as

$$
\begin{aligned}
X_{\tau_{n+1}} & =(1-\varepsilon)\left[X_{\tau_{n}}-Y_{n}-\left(W_{\tau_{n}}-\Phi\left(W_{\tau_{n}}\right)\right)\right] \\
& \times\left[e^{r T_{n}}+I_{n}\left(\Gamma_{n+1}-e^{r T_{n}}\right)\right]+Y_{n+1} .
\end{aligned}
$$

To prevent the problem being trivial, the following condition is imposed :

$$
P\left\{\theta\left(\Gamma_{n+1}-e^{r T_{n}}\right)<0\right\}>0, \quad \theta>0 .
$$

Then it follows that $P\left\{X_{\tau_{n+1}} \geq Y_{n+1}\right\}=1$, of relation ( 5.25) holds if and only if :

$$
X_{\tau_{n}}-Y_{n}-\left(W_{\tau_{n}}-\Phi\left(W_{\tau_{n}}\right)\right)=0, \text { and } \quad V_{\tau_{n}}=0
$$

or,

$$
X_{\tau_{n}}-Y_{n}-\left(W_{\tau_{n}}-\Phi\left(W_{\tau_{n}}\right)\right)>0
$$

and

$$
P\left\{e^{r T_{n}}+I_{n}\left(\Gamma_{n+1}-e^{r T_{n}}\right) \geq 0\right\}=1
$$

These imply that $S\left(X_{\tau_{n}}\right)$ as given by relation (5.23) may be written as

$$
\begin{aligned}
S\left(X_{\tau_{n}}\right) & =\max _{\left\{W_{\tau_{n}}, V_{\tau_{n}}\right\}}\left\{Q_{n}^{\nu} \frac{1}{\gamma}\left(W_{\tau_{n}}-\Phi\left(W_{\tau_{n}}\right)\right)^{\gamma}+A_{n+1}^{-\nu} Q_{n+1}^{\nu} e^{-\delta T_{n}}\right. \\
& \times(1-\varepsilon)^{\gamma}\left[X_{\tau_{n}}-Y_{n}-\left(W_{\tau_{n}}-\Phi\left(W_{\tau_{n}}\right)\right)\right]^{\gamma} \\
& \left.\times E\left[\frac{1}{\gamma}\left[e^{r T_{n}}+I_{n}\left(\Gamma_{n+1}-e^{r T_{n}}\right)\right]^{\gamma}\right]\right\} .
\end{aligned}
$$


Under feasibility with respect to relation ( 5.25), then

$$
S\left(X_{\tau_{n}}\right)=\max \left\{Q_{n}^{\nu} \frac{1}{\gamma}\left(W_{\tau_{n}}-\Phi\left(W_{\tau_{n}}\right)\right)^{\gamma}, \quad \bar{S}\left(X_{\tau_{n}}\right)\right\},
$$

with

$$
\begin{aligned}
\bar{S}\left(X_{\tau_{n}}\right) & =\sup _{\left\{W_{\tau_{n}}, V_{\tau_{n}}\right\}}\left\{Q_{n}^{\nu} \frac{1}{\gamma}\left(W_{\tau_{n}}-\Phi\left(W_{\tau_{n}}\right)\right)^{\gamma}+A_{n+1}^{-\nu} Q_{n+1}^{\nu}\right. \\
& \times(1-\varepsilon)^{\gamma} e^{-\delta T_{n}}\left[X_{\tau_{n}}-Y_{n}-\left(W_{\tau_{n}}-\Phi\left(W_{\tau_{n}}\right)\right)\right]^{\gamma} \\
& \left.\times E\left[\frac{1}{\gamma}\left(e^{r T_{n}}+I_{n}\left(\Gamma_{n+1}-e^{r T_{n}}\right)\right)^{\gamma}\right]\right\},
\end{aligned}
$$

subject to $(5.24),(5.29),(5.30)$ and $I_{n} \geq 0$, since this is equivalent to (5.26) in view of $(5.30)$.

Notice that, the expectation factor in (5.33) may be written as $f\left(I_{n}\right)$, with $f$ is defined by

$$
f(\pi)=E\left[u\left(\exp \left(r T_{n}\right)+\pi\left(\Gamma_{n+1}-\exp \left(r T_{n}\right)\right)\right)\right]
$$

where $u(C)=\frac{1}{\gamma} C^{\gamma}, \quad \gamma \in(0,1)$. Therefore, $\bar{S}\left(X_{\tau_{n}}\right)$ as given by relation ( 5.33) may be expressed as

$$
\begin{aligned}
\bar{S}\left(X_{\tau_{n}}\right) & =\max _{\left\{W_{\tau_{n}}, V_{\tau_{n}}\right\}}\left\{Q_{n}^{\nu} \frac{1}{\gamma}\left(W_{\tau_{n}}-\Phi\left(W_{\tau_{n}}\right)\right)^{\gamma}+A_{n+1}^{-\nu} Q_{n+1}^{\nu}\right. \\
& \left.\times(1-\varepsilon)^{\gamma} e^{-\delta T_{n}}\left[X_{\tau_{n}}-Y_{n}-\left(W_{\tau_{n}}-\Phi\left(W_{\tau_{n}}\right)\right)\right]^{\gamma} f\left(I_{n}\right)\right\} .
\end{aligned}
$$

By Lemma 3.4 and the definition of $\Omega_{n}$, then the maximum of $f\left(I_{n}\right)$ subject to $I_{n} \geq 0$ and $P\left\{e^{r T_{n}}+I_{n}\left(\Gamma_{n+1}-e^{r T_{n}}\right) \geq 0\right\}=1$, is given by $\frac{1}{\gamma} \Omega_{n}$. Then Lemma 3.4 implies that $I_{n}=\pi_{n}$. Therefore, we have

$$
V_{\tau_{n}}=(1-\varepsilon)\left[X_{\tau_{n}}-Y_{n}-\left(W_{\tau_{n}}-\Phi\left(W_{\tau_{n}}\right)\right)\right] \pi_{n}
$$


is optimal and unique for every $W_{\tau_{n}}-\Phi\left(W_{\tau_{n}}\right)$ which satisfies ( 5.24) and ( 5.29) when ( 5.30) holds. It can be shown that it is also optimal when ( 5.28) holds.

Note that, the second term of $\bar{S}\left(X_{\tau_{n}}\right)$ is always non-negative. This implies that $\bar{S}\left(X_{\tau_{n}}\right) \geq Q_{n}^{\nu} \frac{1}{\gamma}\left(W_{\tau_{n}}-\Phi\left(W_{\tau_{n}}\right)\right)^{\gamma}$. By setting $R_{n}=(1-\varepsilon)^{\gamma} e^{-\delta T_{n}} \Omega_{n}$, then $S\left(X_{\tau_{n}}\right)$ of (5.32) reduces to

$$
S\left(X_{\tau_{n}}\right)=\max _{\left\{W_{\tau_{n}}\right\}} S^{W_{\tau_{n}}}\left(X_{\tau_{n}}\right),
$$

with $S^{W_{\tau_{n}}}\left(X_{\tau_{n}}\right)$ is given by

$$
\begin{aligned}
S^{W_{\tau_{n}}}\left(X_{\tau_{n}}\right) & =Q_{n}^{\nu} \frac{1}{\gamma}\left(W_{\tau_{n}}-\Phi\left(W_{\tau_{n}}\right)\right)^{\gamma}+A_{n+1}^{-\nu} Q_{n+1}^{\nu} R_{n} \\
& \times \frac{1}{\gamma}\left[X_{\tau_{n}}-Y_{n}-\left(W_{\tau_{n}}-\Phi\left(W_{\tau_{n}}\right)\right)\right]^{\gamma} .
\end{aligned}
$$

The utility function $u$ is strictly concave and $u^{\prime}(0)=\infty$. These imply that $S^{W_{\tau_{n}}}$ is differentiable with respect to $W_{\tau_{n}}$. By differentiation of $S^{W_{\tau_{n}}}$ with respect to $W_{\tau_{n}}$, then we have

$$
\begin{aligned}
& \frac{d S^{W_{\tau_{n}}}}{d W_{\tau_{n}}}=Q_{n}^{\nu}\left(W_{\tau_{n}}-\Phi\left(W_{\tau_{n}}\right)\right)^{-\nu}\left(1-\Phi^{\prime}\left(W_{\tau_{n}}\right)\right)+A_{n+1}^{-\nu} Q_{n+1}^{\nu} R_{n} \\
& \quad \times\left[X_{\tau_{n}}-Y_{n}-\left(W_{\tau_{n}}-\Phi\left(W_{\tau_{n}}\right)\right)\right]^{-\nu}\left[-d Y_{n} / d W_{\tau_{n}}-\left(1-\Phi^{\prime}\left(W_{\tau_{n}}\right)\right] .\right.
\end{aligned}
$$

from the definition of $Y_{n}$ as in 5.19), we have that $d Y_{n} / d W_{\tau_{n}}=\Phi^{\prime}\left(W_{\tau_{n}}\right)$. Set $d S^{W_{\tau_{n}}} / d W_{\tau_{n}}=0$, and re-arrange the terms to get

$$
\begin{aligned}
& {\left[W_{\tau_{n}}-\Phi\left(W_{\tau_{n}}\right)\right]^{-\nu}} \\
& \quad=Q_{n}^{-\nu} A_{n+1}^{-\nu} Q_{n+1}^{\nu} R_{n}\left(1-\Phi^{\prime}\left(W_{\tau_{n}}\right)\right)^{-1}\left[X_{\tau_{n}}-Y_{n}-\left(W_{\tau_{n}}-\Phi\left(W_{\tau_{n}}\right)\right)\right]^{-\nu} .
\end{aligned}
$$

This implies that

$$
\begin{aligned}
& {\left[W_{\tau_{n}}-\Phi\left(W_{\tau_{n}}\right)\right]} \\
& \quad=A_{n+1} Q_{n} Q_{n+1}^{-1} R_{n}^{-1 / \nu}\left(1-\Phi^{\prime}\left(W_{\tau_{n}}\right)\right)^{1 / \nu}\left[X_{\tau_{n}}-Y_{n}-\left(W_{\tau_{n}}-\Phi^{\prime}\left(W_{\tau_{n}}\right)\right)\right],
\end{aligned}
$$


which gives

$$
\begin{gathered}
{\left[W_{\tau_{n}}-\Phi\left(W_{\tau_{n}}\right)\right]\left[1+\frac{A_{n+1} Q_{n}\left(1-\Phi^{\prime}\left(W_{\tau_{n}}\right)\right)^{1 / \nu}}{Q_{n+1} R_{n}^{1 / \nu}}\right]} \\
=\frac{A_{n+1} Q_{n}\left(1-\Phi^{\prime}\left(W_{\tau_{n}}\right)\right)^{1 / \nu}}{Q_{n+1} R_{n}^{1 / \nu}}\left(X_{\tau_{n}}-Y_{n}\right) .
\end{gathered}
$$

Let $F_{n}$ be defined by

$$
F_{n}=\frac{A_{n+1} Q_{n}\left(1-\Phi^{\prime}\left(W_{\tau_{n}}\right)\right)^{1 / \nu}}{A_{n+1} Q_{n}\left(1-\Phi^{\prime}\left(W_{\tau_{n}}\right)\right)^{1 / \nu}+Q_{n+1} R_{n}^{1 / \nu}} .
$$

Then the optimal withdrawal process $W_{\tau_{n}}$ is given by

$$
W_{\tau_{n}}=F_{n}\left(X_{\tau_{n}}-Y_{n}\right)+\Phi\left(W_{\tau_{n}}\right)
$$

By insertion of the optimal withdrawal process $W_{\tau_{n}}$ as given by relation ( 5.38) into relation ( 5.35) then the optimal investment process $V_{\tau_{n}}$ is given by

$$
V_{\tau_{n}}=(1-\varepsilon)\left(1-F_{n}\right)\left(X_{\tau_{n}}-Y_{n}\right) \pi_{n}
$$

Finally, by substitution of the optimal withdrawal process $W_{\tau_{n}}$ into $S\left(X_{\tau_{n}}\right)$ as given by relation (5.36) yields

$$
\begin{aligned}
S\left(X_{\tau_{n}}\right) & =Q_{n}^{\nu} F_{n}^{\gamma} \frac{1}{\gamma}\left(X_{\tau_{n}}-Y_{n}\right)^{\gamma}+A_{n+1}^{-\nu} Q_{n+1}^{\nu} R_{n} \\
& \times \frac{1}{\gamma}\left(X_{\tau_{n}}-Y_{n}-F_{n}\left(X_{\tau_{n}}-Y_{n}\right)\right)^{\gamma} \\
& =Q_{n}^{\nu} A_{n}^{-\nu} \frac{1}{\gamma}\left(X_{\tau_{n}}-Y_{n}\right)^{\gamma}\left\{A_{n}^{\nu} F_{n}^{\gamma}\right. \\
& \left.+A_{n}^{\nu} A_{n+1}^{-\nu} Q_{n}^{-\nu} Q_{n+1}^{\nu} R_{n}\left(1-F_{n}\right)^{\gamma}\right\} .
\end{aligned}
$$

Then this implies that $S\left(X_{\tau_{n}}\right)=U\left(X_{\tau_{n}}\right)$ if and only if

$$
A_{n}^{\nu} F_{n}^{\gamma}+A_{n}^{\nu} A_{n+1}^{-\nu} Q_{n}^{-\nu} Q_{n+1}^{\nu} R_{n}\left(1-F_{n}\right)^{\gamma}=1 .(\star)
$$


By replacing $F_{n}$ with the right hand side of $(5.18)$, then $(\star)$ holds if the following holds :

$$
\begin{aligned}
& {\left[A_{n+1} Q_{n}\left(1-\Phi^{\prime}\left(W_{\tau_{n}}\right)\right)^{1 / \nu}+Q_{n+1} R_{n}^{1 / \nu}\right]^{\gamma}=} \\
& \quad A_{n}^{\nu} A_{n+1}^{-\nu} Q_{n}^{-\nu}\left[A_{n+1} Q_{n}\left(1-\Phi^{\prime}\left(W_{\tau_{n}}\right)\right)^{\gamma / \nu}+Q_{n+1}^{\nu} R_{n}\right] .
\end{aligned}
$$

This implies that $S\left(X_{\tau_{n}}\right)=U\left(X_{\tau_{n}}\right)$ if and only if

$$
A_{n}=\frac{A_{n+1} Q_{n}\left[A_{n+1} Q_{n}\left(1-\Phi^{\prime}\left(W_{\tau_{n}}\right)\right)^{1 / \nu}+Q_{n+1} R_{n}^{1 / \nu}\right]^{\gamma / \nu}}{\left[A_{n+1} Q_{n}\left(1-\Phi^{\prime}\left(W_{\tau_{n}}\right)\right)^{\gamma / \nu}+Q_{n+1} R_{n}^{1 / \nu}\right]^{1 / \nu}} .
$$

Therefore, the proof of Theorem 5.1 has been completed

The following is similar to those of Remark 4.2.

Remark 5.1 Suppose that the optimal control problem be defined by the problem (5.10)- (5.11), and let $T_{n}$ be fixed for $n=1,2,3, \ldots$ Then $F_{n}$ as defined by (5.18) has a property that either $F_{n} \geq Q_{n}\left(1-R_{n}^{1 / \nu}\right)$, or $F_{n}<Q_{n}\left(1-R_{n}^{1 / \nu}\right)$

Remark 5.2 Let $A_{n+1}$ satisfying relation (5.18). If $F_{n}$ as given in relation ( 5.18) has a property such that $F_{n} \geq Q_{n}\left(1-R_{n}^{1 / \nu}\right)$, then $A_{n+1}$ is such that

$$
\frac{A_{n+1}}{Q_{n+1}} \geq \frac{\left(1-R_{n}^{1 / \nu}\right) R_{n}^{1 / \nu}}{\left(1-\Phi^{\prime}\left(W_{\tau_{n}}\right)\right)^{1 / \nu}}
$$

Proof : By applying $F_{n} \geq Q_{n}\left(1-R_{n}^{1 / \nu}\right)$ in relation ( 5.18) and arranging all terms, then $A_{n+1}$ satisfy

$$
\begin{aligned}
\frac{A_{n+1}}{Q_{n+1}} & =\frac{A_{n} R_{n}^{1 / \nu}}{Q_{n}\left(1-F_{n}\right)\left(1-\Phi^{\prime}\left(W_{\tau_{n}}\right)\right)^{1 / \nu}} \\
& \geq \frac{Q_{n}\left(1-R_{n}^{1 / \nu}\right) R_{n}^{1 / \nu}}{Q_{n}\left(1-F_{n}\right)\left(1-\Phi^{\prime}\left(W_{\tau_{n}}\right)\right)^{1 / \nu}} \\
& \geq \frac{\left(1-R_{n}^{1 / \nu}\right) R_{n}^{1 / \nu}}{\left[1-Q_{n}\left(1-R_{n}^{1 / \nu}\right)\right]\left(1-\Phi^{\prime}\left(W_{\tau_{n}}\right)\right)^{1 / \nu}}
\end{aligned}
$$


Since $0 \leq Q_{n} \leq 1$. Also $R_{n} \leq(1-\varepsilon)^{\gamma / \nu} \leq 1$, as $\varepsilon<1$, then

$$
\frac{\left(1-R_{n}^{1 / \nu}\right) R_{n}^{1 / \nu}}{\left[1-Q_{n}\left(1-R_{n}^{1 / \nu}\right)\right]\left(1-\Phi^{\prime}\left(W_{\tau_{n}}\right)\right)^{1 / \nu}} \geq \frac{\left(1-R_{n}^{1 / \nu}\right) R_{n}^{1 / \nu}}{\left(1-\Phi^{\prime}\left(W_{\tau_{n}}\right)\right)^{1 / \nu}}
$$

Hence,

$$
\frac{A_{n+1}}{Q_{n+1}} \geq \frac{\left(1-R_{n}^{1 / \nu}\right) R_{n}^{1 / \nu}}{\left(1-\Phi^{\prime}\left(W_{\tau_{n}}\right)\right)^{1 / \nu}}
$$

Remark 5.3 If at any $\tau_{n}, \quad \Phi\left(W_{\tau_{n}}\right)=W_{\tau_{n}}$, then the implications are as follows :

1. $Y_{n}=X_{\tau_{n}}$, by $(5.15)$.

2. $T_{n}$ can be computed from ( 5.19) as

$$
0<T_{n}=\frac{1}{r} \ln \left[\frac{X_{\tau_{n+1}}}{\left(X_{\tau_{n}}-W_{\tau_{n}}\right)(1-\varepsilon)}\right]<\infty .
$$

3. $U\left(X_{\tau_{n}}\right)=0$, implying by (5.2) that $C_{t}=0$, almost every where for $t \geq \tau_{n}$. This can occur only for utility function which satisfy $u(0)=0$.

4. Case 2 and 3 imply that $W_{\tau_{n}}>\Phi\left(W_{\tau_{n}}\right)$ always in the case of infinitetime horizon

Corollary 5.2 It is possible that $\Phi\left(W_{\tau_{n}}\right)=W_{\tau_{n}}$, in which case

$$
T_{n}=\frac{1}{r} \ln \left[\frac{X_{\tau_{n+1}}}{\left(X_{\tau_{n}}-W_{\tau_{n}}\right)(1-\varepsilon)}\right] .
$$

This imply that $\Phi\left(W_{\tau_{n}}\right)<W_{\tau_{n}}$ if the specified $T_{n}$ are not given by (5.40). 


\subsection{Optimal Transaction Intervals}

The main aim of Section 5.5 is to find the optimal transaction times for an investor who faces the optimal control problem as given by the problem (5.10)-( 5.11). Since finding the optimal transaction times is equivalent to finding the optimal transaction intervals, then Section 5.5 is devoted to finding the optimal transaction interval $T_{n}$, for $n=1,2,3, \ldots$ This is done by deriving an equation satisfied by the interval of time $T_{n}$. Later, confirmation of a solution to that equation, which is the optimal choice for $T_{n}$, is given.

Let recall the value function $U\left(X_{\tau_{n}}\right)$ of relation (5.10) which is given by

$$
U\left(X_{\tau_{n}}\right)=\max _{\left\{T_{n}, W_{\tau_{n}}, V_{\tau_{n}}\right\}}\left\{Q_{n}^{\nu} \frac{1}{\gamma}\left[W_{\tau_{n}}-\Phi\left(W_{\tau_{n}}\right)\right]^{\gamma}+e^{-\delta T_{n}} E\left[U\left(X_{\tau_{n+1}}\right) \mid \mathcal{H}_{\tau_{n}}\right]\right\} .
$$

By differentiations of the value function $U\left(X_{\tau_{n}}\right)$ with respect to $W_{\tau_{n}}, V_{\tau_{n}}$ and $T_{n}$, respectively, and set each of these derivatives equal to zero, then we get the following necessary conditions for the value function $U$, and for $n=1,2,3, \ldots$ :

$$
\begin{gathered}
Q_{n}^{\nu}\left[W_{\tau_{n}}-\Phi\left(W_{\tau_{n}}\right)\right]^{-\nu}\left(1-\Phi^{\prime}\left(W_{\tau_{n}}\right)\right)=(1-\varepsilon) e^{-(\delta-r) T_{n}} E\left[U^{\prime}\left(X_{\tau_{n+1}}\right) \mid \mathcal{H}_{\tau_{n}}\right] \\
E\left[\left(\Gamma_{n+1}-e^{r T_{n}}\right) U^{\prime}\left(X_{\tau_{n+1}}\right) \mid \mathcal{H}_{\tau_{n}}\right]=0 \\
\delta e^{-\frac{\delta}{\nu} T_{n}} Q_{n}^{-\gamma} \frac{1}{\gamma}\left[W_{\tau_{n}}-\Phi\left(W_{\tau_{n}}\right)\right]^{\gamma}= \\
\delta e^{-\delta T_{n}} E\left[U\left(X_{\tau_{n+1}}\right) \mid \mathcal{H}_{\tau_{n}}\right]-e^{\delta T_{n}} \frac{\partial E\left[U\left(X_{\tau_{n+1}}\right) \mid \mathcal{H}_{\tau_{n}}\right]}{\partial T_{n}} .
\end{gathered}
$$


Applications of Itốs formula ${ }^{1}$ on the value function $U\left(X_{\tau_{n}}\right)$, results in

$$
U\left(X_{\tau_{n+1}}\right)-U\left(X_{\tau_{n}}\right)=\int_{\tau_{n}+}^{\tau_{n+1}}\left[U^{\prime}\left(X_{t}\right) d X_{t}+\frac{1}{2} U^{\prime \prime}\left(X_{t}\right) d X_{t}^{2}\right] .
$$

From the total wealth equation of (5.9), for any $t \in\left[\tau_{n}, \tau_{n+1}\right)$, we have

$$
X_{t}=(1-\varepsilon)\left[X_{\tau_{n}}-W_{\tau_{n}}\right] e^{r\left(t-\tau_{n}\right)}+V_{\tau_{n}}\left(\Gamma_{t}-e^{r\left(t-\tau_{n}\right)}\right),
$$

with return from risky investment is given by

$$
\Gamma_{t}=\exp \left[\left(\alpha-\frac{1}{2} \sigma^{2}\right)\left(t-\tau_{n}\right)+\sigma\left(B_{t}-B_{\tau_{n}}\right)\right]
$$

This implies that for any $t \in\left[\tau_{n}, \tau_{n+1}\right)$, we have

$$
d X_{t}=\left[r\left(X_{t}-G_{t}\right)+\alpha G_{t}\right] d t+\sigma G_{t} d B_{t}, \quad \text { and } \quad d X_{t}^{2}=\sigma^{2} G_{t}^{2} d t
$$

with $G_{t} \equiv V_{\tau_{n}} \Gamma_{t}$. By substitutions of $d X_{t}$ and $d X_{t}^{2}$ respectively, into (5.42) yields

$$
\begin{aligned}
U\left(X_{\tau_{n+1}}\right)-U\left(X_{\tau_{n}}\right) & =\int_{\tau_{n}+}^{\tau_{n+1}}\left\{\left[r\left(X_{t}-G_{t}\right)+\alpha G_{t}\right] U^{\prime}\left(X_{t}\right) d t\right. \\
& \left.+\frac{1}{2} \sigma^{2} G_{t}^{2} U^{\prime \prime}\left(X_{t}\right) d t+\sigma G_{t} U^{\prime}\left(X_{t}\right) d B_{t}\right\}
\end{aligned}
$$

Let processes $\left\{Z_{t}\right\}$ be defined by

$$
Z_{t}=\int_{\tau_{n}}^{t} \sigma G_{s} U^{\prime}\left(X_{s}\right) d B_{s}, \quad t \in\left(\tau_{n}, \tau_{n+1}\right]
$$

Then processes $\left\{Z_{t}\right\}$ is a martingale. ${ }^{2}$ This implies that

$$
\begin{aligned}
\frac{\partial E\left[U\left(X_{\tau_{n+1}}\right) \mid \mathcal{H}_{\tau_{n}}\right]}{\partial T_{n}} & =E\left[\left[r\left(X_{\tau_{n+1}}-G_{\tau_{n+1}}\right)+\alpha G_{\tau_{n+1}}\right] U^{\prime}\left(X_{\tau_{n+1}}\right)\right. \\
& \left.+\frac{1}{2} \sigma^{2} G_{\tau_{n+1}}^{2} U^{\prime \prime}\left(X_{\tau_{n+1}}\right) \mid \mathcal{H}_{\tau_{n}}\right]
\end{aligned}
$$

\footnotetext{
${ }^{1}$ For details, see Theorem 32 of [22]
}

${ }^{2}$ See, appendix 3 of [7]) 
with $G_{\tau_{n+1}} \equiv V_{\tau_{n}} \Gamma_{n+1}$. Then by substitution of ( 5.44) into ( 5.41) results in

$$
\begin{aligned}
& \delta e^{-\frac{\delta}{\nu} T_{n}} Q_{n}^{-\gamma} \frac{1}{\gamma}\left[W_{\tau_{n}}-\Phi\left(W_{\tau_{n}}\right)\right]^{\gamma}= \\
& \delta e^{-\delta T_{n}} E\left[U\left(X_{\tau_{n+1}}\right) \mid \mathcal{H}_{\tau_{n}}\right]-e^{-\delta T_{n}} E\left[\left[r\left(X_{\tau_{n+1}}-G_{\tau_{n+1}}\right)\right.\right. \\
& \left.\left.\quad+\alpha G_{\tau n+1}\right] U^{\prime}\left(X_{\tau_{n+1}}\right)+\frac{1}{2} \sigma^{2} G_{\tau_{n+1}}^{2} U^{\prime \prime}\left(X_{\tau_{n+1}}\right) \mid \mathcal{H}_{\tau_{n}}\right] .
\end{aligned}
$$

By applications of $W_{\tau_{n}}$ and $V_{\tau_{n}}$ as given by relation ( 5.15) and (5.16), respectively into the total wealth process $X_{\tau_{n+1}}$ as in relation (5.9), results in

$$
X_{\tau_{n+1}}=(1-\varepsilon)\left(1-F_{n}\right)\left(X_{\tau_{n}}-Y_{n}\right)\left[e^{r T_{n}}+\pi_{n}\left(\Gamma_{n+1}-e^{r T_{n}}\right)\right]+Y_{n+1}
$$

Since $G_{\tau_{n+1}}=V_{\tau_{n}} \Gamma_{n+1}$, with $\quad \Gamma_{n+1} \equiv \Gamma_{\tau_{n+1}}$, and $V_{\tau_{n}}$ as given by relation (5.16), then we have

$$
G_{\tau_{n+1}}=(1-\varepsilon)\left(1-F_{n}\right)\left(X_{\tau_{n}}-Y_{n}\right) \pi_{n} \Gamma_{n+1} .
$$

Based on the value function $U\left(X_{\tau_{n}}\right)$ of relation ( 5.10) and the total wealth process $X_{\tau_{n+1}}$ as given by relation (5.46), result in the following :

$$
\begin{aligned}
E\left[U\left(X_{\tau_{n+1}}\right) \mid \mathcal{H}_{\tau_{n}}\right] & =Q_{n+1}^{\nu} A_{n+1}^{-\nu}(1-\varepsilon)^{\gamma}\left(1-F_{n}\right)^{\gamma} \frac{1}{\gamma}\left(X_{\tau_{n}}-Y_{n}\right)^{\gamma} \\
& \times E\left(\left[e^{r T_{n}}+\pi_{n}\left(\Gamma_{n+1}-e^{r T_{n}}\right)\right]^{\gamma}\right) \\
E\left[U^{\prime}\left(X_{\tau_{n+1}}\right) \mid \mathcal{H}_{\tau_{n}}\right] & =Q_{n+1}^{\nu} A_{n+1}^{-\nu}(1-\varepsilon)^{-\nu}\left(1-F_{n}\right)^{-\nu}\left(X_{\tau_{n}}-Y_{n}\right)^{-\nu} \\
& \times E\left(\left[e^{r T_{n}}+\pi_{n}\left(\Gamma_{n+1}-e^{r T_{n}}\right)\right]^{-\nu}\right) \\
E\left[U^{\prime \prime}\left(X_{\tau_{n+1}}\right) \mid \mathcal{H}_{\tau_{n}}\right] & =Q_{n+1}^{\nu} A_{n+1}^{-\nu}(1-\varepsilon)^{-\nu-1}\left(1-F_{n}\right)^{-\nu-1}\left(X_{\tau_{n}}-Y_{n}\right)^{-\nu-1} \\
& \times(-\nu) E\left(\left[e^{r T_{n}}+\pi_{n}\left(\Gamma_{n+1}-e^{r T_{n}}\right)\right]^{-\nu-1}\right)
\end{aligned}
$$


From the definition of $\Omega_{n}$ in relation (5.20), then its derivative with respect to $\pi_{n}$ gives

$$
\left.E\left(\left[e^{r T_{n}}+\pi_{n}\left(\Gamma_{n+1}-e^{r T_{n}}\right)\right]^{-\nu}\left[\Gamma_{n+1}-e^{r T_{n}}\right)\right]\right)=0 .
$$

Then its implications are :

$$
\begin{aligned}
\Omega_{n} & =E\left(e^{r T_{n}}\left[e^{r T_{n}}+\pi_{n}\left(\Gamma_{n+1}-e^{r T_{n}}\right)\right]^{-\nu}\right), \\
& =E\left(\Gamma_{n+1}\left[e^{r T_{n}}+\pi_{n}\left(\Gamma_{n+1}-e^{r T_{n}}\right)\right]^{-\nu}\right), \\
& =E\left(\Gamma_{n+1}^{2}\left[e^{r T_{n}}+\pi_{n}\left(\Gamma_{n+1}-e^{r T_{n}}\right)\right]^{-\nu-1}\right) .
\end{aligned}
$$

By applications of ( 5.15), ( 5.46),( 5.47), ( 5.48),( 5.49), ( 5.50),( 5.51), (5.52), (5.53) in the relation (5.45), results in

$$
\begin{aligned}
\delta e^{-\frac{\delta}{\nu} T_{n}} Q_{n}^{-\gamma} F_{n}^{\gamma} \frac{1}{\gamma}\left(X_{\tau_{n}}-Y_{n}\right)^{\gamma}=Q_{n+1}^{\nu} A_{n+1}^{-\nu}\left(1-F_{n}\right)^{\gamma} R_{n} \\
\quad \times \quad\left(X_{\tau_{n}}-Y_{n}\right)^{\gamma}\left[-r-(\alpha-r) \pi_{n}+\frac{\delta}{\gamma}+\frac{1}{2} \sigma^{2}(1-\gamma) \pi_{n}^{2}\right] \\
-r Q_{n+1}^{\nu} A_{n+1}^{-\nu}(1-\varepsilon)^{-1} e^{-r T_{n}} R_{n}\left(1-F_{n}\right)^{-\nu}\left(X_{\tau_{n}}-Y_{n}\right)^{-\nu} Y_{n+1} .
\end{aligned}
$$

By substitutions of $F_{n}$ as given by relation ( 5.18 ) into the last equation yields

$$
\begin{aligned}
& \left(X_{\tau_{n}}-Y_{n}\right)\left\{\delta e^{-\frac{\delta}{\nu} T_{n}} \frac{\left[A_{n+1}\left(1-\Phi^{\prime}\left(W_{\tau_{n}}\right)\right)^{\nu}\right]^{\gamma}}{\left[A_{n+1} Q_{n}\left(1-\Phi^{\prime}\left(W_{\tau_{n}}\right)\right)^{\nu}+Q_{n+1} R_{n}^{1 / \nu}\right]^{\gamma}}\right. \\
& \quad+Q_{n+1}^{\nu} R_{n} \frac{\left[Q_{n+1} R_{n}^{1 / \nu}\right]^{\gamma}}{\left[A_{n+1} Q_{n}\left(1-\Phi^{\prime}\left(W_{\tau_{n}}\right)\right)^{\nu}+Q_{n+1} R_{n}^{1 / \nu}\right] \gamma} \\
& \left.\quad \times\left[-\delta+\gamma r+\gamma(\alpha-r) \pi_{n}-\frac{1}{2} \sigma^{2} \gamma(1-\gamma) \pi_{n}^{2}\right]\right\} \\
& \quad+\frac{\gamma r Y_{n+1} R_{n} Q_{n+1}^{\nu}}{A_{n+1}^{\nu}(1-\varepsilon) e^{r T_{n}}} \frac{\left[Q_{n+1} R_{n}^{1 / \nu}\right]^{-\nu}}{\left[A_{n+1} Q_{n}\left(1-\Phi^{\prime}\left(W_{\tau_{n}}\right)\right)^{\nu}+Q_{n+1} R_{n}^{1 / \nu}\right]^{-\nu}}=0 .
\end{aligned}
$$


Finally, by dividing all terms of the last equation by

$$
\frac{A_{n+1}^{-\nu}}{\left[A_{n+1} Q_{n}\left(1-\Phi^{\prime}\left(W_{\tau_{n}}\right)\right)^{\nu}+Q_{n+1} R_{n}^{1 / \nu}\right]^{\gamma}}
$$

results in the following equation :

$$
g\left(T_{n}\right)+\left(X_{\tau_{n}}-Y_{n}\right) h\left(T_{n}\right)=0,
$$

with functions $g$ and $h$ are defined as the following:

$$
\begin{aligned}
g\left(T_{n}\right)= & \frac{\gamma r Y_{n+1}}{(1-\varepsilon) e^{r T_{n}}}\left[A_{n+1} Q_{n}\left(1-\Phi^{\prime}\left(W_{\tau_{n}}\right)\right)^{1 / \nu}+Q_{n+1} R_{n}^{1 / \nu}\right] \\
h\left(T_{n}\right) & =\delta e^{-\frac{\delta}{\nu} T_{n}} A_{n+1}\left(1-\Phi^{\prime}\left(W_{\tau_{n}}\right)\right)^{\gamma / \nu}+Q_{n+1} R_{n}^{1 / \nu}[-\delta+\gamma r \\
& \left.+\gamma(\alpha-r) \pi_{n}-\frac{1}{2} \sigma^{2} \gamma(1-\gamma) \pi_{n}^{2}\right]
\end{aligned}
$$

Therefore, the proof of the following Theorem has been completed.

Theorem 5.2 Let the optimal control problem for an investor be defined by the problem (5.10) subject to (5.11). Then the optimal transaction interval of times $T_{n}, \quad n=1,2,3, \ldots$, satisfy

$$
g\left(T_{n}\right)+\left(X_{\tau_{n}}-Y_{n}\right) h\left(T_{n}\right)=0,
$$

where $g$ and $h$ are given by

$$
\begin{aligned}
g\left(T_{n}\right)= & \frac{\gamma r Y_{n+1}}{(1-\varepsilon) e^{r T_{n}}}\left[A_{n+1} Q_{n}\left(1-\Phi^{\prime}\left(W_{\tau_{n}}\right)\right)^{1 / \nu}+Q_{n+1} R_{n}^{1 / \nu}\right] \\
h\left(T_{n}\right) & =\delta e^{-\frac{\delta}{\nu} T_{n}} A_{n+1}\left(1-\Phi^{\prime}\left(W_{\tau_{n}}\right)\right)^{\gamma / \nu}+Q_{n+1} R_{n}^{1 / \nu}[-\delta+\gamma r \\
& \left.+\gamma(\alpha-r) \pi_{n}-\frac{1}{2} \sigma^{2} \gamma(1-\gamma) \pi_{n}^{2}\right]
\end{aligned}
$$


Theorem 5.3 Suppose that $\alpha, \delta, \varepsilon, \gamma, \Phi^{\prime}, r, \sigma$ satisfying the following conditions :

1. $\alpha-r \geq \frac{1}{2} \sigma^{2}(1-\gamma)$.

2. $\max (\gamma \alpha, \gamma r)<\delta<\min \left(\frac{\gamma r\left(1-\Phi^{\prime}\left(W_{\tau_{n}}\right)\right)}{(1-\varepsilon)^{\gamma / \nu}}, r-\gamma r\right)$.

If $F_{n}$ as given by (5.18) has the property such that $F_{n} \geq Q_{n}\left(1-R_{n}^{1 / \nu}\right)$, Then the relation

$$
g\left(T_{n}\right)+\left(X_{\tau_{n}}-Y_{n}\right) h\left(T_{n}\right)=0
$$

as defined in Theorem 5.2 has a solution.

Proof : Let $H\left(T_{n}\right)=g\left(T_{n}\right)+\left(X_{\tau_{n}}-Y_{n}\right) h\left(T_{n}\right)$, where $g$ and $h$ are defined by relation ( 5.58) and (5.59) respectively. It will be shown that $H\left(T_{n}\right)=0$ has a solution. Note that, functions $g$ and $h$, hence $H$, are continuous in $T_{n}$. Then it will be shown that $H\left(T_{n}\right)>0$ as $T_{n} \rightarrow 0^{+}$and $H\left(T_{n}\right)<0$ for large $T_{n}$

By making use of $g$ and $h$ as given by relations ( 5.58) and (5.59), then we have

$$
\begin{aligned}
\lim _{T_{n} \rightarrow 0^{+}} H\left(T_{n}\right) & =\lim _{T_{n} \rightarrow 0^{+}}\left[g\left(T_{n}\right)+\left(X_{\tau_{n}}-Y_{n}\right) h\left(T_{n}\right)\right] \\
& =\lim _{T_{n} \rightarrow 0^{+}} \frac{\gamma r Y_{n+1}}{(1-\varepsilon) e^{r T_{n}}}\left[A_{n+1} Q_{n}\left(1-\Phi^{\prime}\left(W_{\tau_{n}}\right)\right)^{1 / \nu}+Q_{n+1} R_{n}^{1 / \nu}\right] \\
& +\lim _{T_{n} \rightarrow 0^{+}} Q_{n+1}\left(X_{\tau_{n}}-Y_{n}\right)\left\{\delta e^{-\delta / \nu T_{n}} \frac{A_{n+1}}{Q_{n+1}}\left(1-\Phi^{\prime}\left(W_{\tau_{n}}\right)\right)^{\gamma / \nu}\right. \\
& \left.+R_{n}^{1 / \nu}\left[-\delta+\gamma r+\gamma(\alpha-r) \pi_{n}-1 / 2 \sigma^{2} \gamma(1-\gamma) \pi_{n}^{2}\right]\right\} .
\end{aligned}
$$

Since by relation $(5.19) \quad Y_{n}=\Phi\left(W_{\tau_{n}}\right)+(1-\varepsilon)^{-1} e^{-r T_{n}} Y_{n+1}$, and by 
Remark $5.2 \frac{A_{n+1}}{Q_{n+1}} \geq \frac{R_{n}\left(1-R_{n}^{1 / \nu}\right)}{\left(1-\Phi^{\prime}\left(W_{\tau_{n}}\right)\right)^{1 / \nu}}$, for $F_{n} \geq Q_{n}\left(1-R_{n}^{1 / \nu}\right)$, then results in

$$
\begin{aligned}
\lim _{T_{n} \rightarrow 0^{+}} H\left(T_{n}\right) & \geq \lim _{T_{n} \rightarrow 0^{+}} \frac{\gamma r Y_{n+1}\left[A_{n+1} Q_{n}\left(1-\Phi^{\prime}\left(W_{\tau_{n}}\right)\right)^{1 / \nu}+Q_{n+1} R_{n}^{1 / \nu}\right]}{(1-\varepsilon) e^{r T_{n}}} \\
& +\lim _{T_{n} \rightarrow 0^{+}} Q_{n+1}\left(X_{\tau_{n}}-\Phi\left(W_{\tau_{n}}\right)-(1-\varepsilon)^{-1} e^{-r T_{n}} Y_{n+1}\right) \\
& \times\left\{\delta e^{-\delta / \nu T_{n}} \frac{R_{n}^{1 / \nu}\left(1-R_{n}^{1 / \nu}\right)}{\left(1-\Phi^{\prime}\left(W_{\tau_{n}}\right)\right)}\right. \\
& \left.+R_{n}^{1 / \nu}\left[-\delta+\gamma r+\gamma(\alpha-r) \pi_{n}-1 / 2 \sigma^{2} \gamma(1-\gamma) \pi_{n}^{2}\right]\right\} .
\end{aligned}
$$

By assumption of the theorem we have that $(\alpha-r) \geq \frac{1}{2} \sigma^{2}(1-\gamma)$. From relation (5.20) we also have that $0 \leq \pi_{n} \leq 1$. Therefore, we have

$$
-\delta+\gamma r+\gamma(\alpha-r) \pi_{n}-1 / 2 \sigma^{2} \gamma(1-\gamma) \pi_{n}^{2} \geq-\delta+\gamma r .
$$

This implies that

$$
\begin{aligned}
\lim _{T_{n} \rightarrow 0^{+}} H\left(T_{n}\right) & \geq \gamma r(1-\varepsilon)^{-1} Y_{n+1} Q_{n+1}(1-\varepsilon)^{\gamma / \nu} \\
& +(1-\varepsilon)^{\gamma / \nu} Q_{n+1}\left(X_{\tau_{n}}-\Phi\left(W_{\tau_{n}}\right)-(1-\varepsilon)^{-1} Y_{n+1}\right) \\
& \times\left\{\delta\left[1-(1-\varepsilon)^{\gamma / \nu}\right]\left(1-\Phi^{\prime}\left(W_{\tau_{n}}\right)\right)^{\gamma / \nu}+[-\delta+\gamma r]\right\} .
\end{aligned}
$$

By assumption of theorem we have that $\delta<\frac{\gamma r\left(1-\Phi^{\prime}\left(W_{\tau_{n}}\right)\right)}{(1-\varepsilon)^{\gamma / \nu}}$. This implies that

$$
\lim _{T_{n} \rightarrow 0^{+}} H\left(T_{n}\right)>\gamma r(1-\varepsilon)^{-1} Y_{n+1} Q_{n+1}(1-\varepsilon)^{\gamma / \nu} .
$$

Since $\varepsilon, \gamma, Q_{n+1}, Y_{n+1}$, are nonnegatives, then

$$
\lim _{T_{n} \rightarrow 0^{+}} H\left(T_{n}\right)>0 .
$$

On the other hand, since $A_{n+1}, \quad Q_{n+1}, \quad Q_{n}$ are nonnegatives and less than one, then

$$
\begin{aligned}
H\left(T_{n}\right) & \leq \frac{\gamma r}{(1-\varepsilon) e^{r T_{n}}} Y_{n+1}\left[\left(1-\Phi^{\prime}\left(W_{\tau_{n}}\right)\right)^{1 / \nu}+(1-\varepsilon)^{\gamma / \nu}\right] \\
& +\left(X_{\tau_{n}}-Y_{n}\right)\left\{\delta e^{-\frac{\delta}{\nu} T_{n}}+Q_{n+1} R_{n}^{1 / \nu}[-\delta+\max (\gamma \alpha, \gamma r)]\right\}
\end{aligned}
$$


By arranging all terms, then we have that

$$
\begin{aligned}
H\left(T_{n}\right) & \leq e^{-\frac{\delta}{\nu} T_{n}}\left(X_{\tau_{n}}-Y_{n}\right)\left\{\frac{\gamma r}{(1-\varepsilon) e^{r T_{n}}\left(X_{\tau_{n}}-Y_{n}\right)} e^{\frac{\delta}{\nu} T_{n}} Y_{n+1}\right. \\
& \left.\left.+\delta+e^{\delta / \nu T_{n}} Q_{n+1} R_{n}^{1 / \nu}[-\delta+\max (\gamma \alpha, \gamma r)]\right\}\right\} .
\end{aligned}
$$

Since, by assumptions of Theorem 5.3 that $\delta>\max (\gamma \alpha, \gamma r)$, and $r>\delta / \nu$, then $H\left(T_{n}\right)<0$ for large $T_{n}$.

It has been shown that $H\left(T_{n}\right)>0$ as $T_{n} \rightarrow 0^{+}$and $H\left(T_{n}\right)<0$ for large $T_{n}$. Since $H\left(T_{n}\right)$ is continuous in $T_{n}$, then there exists $\hat{T}_{n} \quad \forall n$, such that $H\left(\hat{T}_{n}\right)=0$. Hence, the proof of Theorem 5.3 has been completed

Theorem 5.4 Suppose that $\alpha, \delta, \varepsilon, \gamma, \Phi^{\prime}, r, \sigma$ satisfying the following conditions :

1. $\alpha-r \geq \frac{1}{2} \sigma^{2}(1-\gamma)$

2. $\max (\gamma \alpha, \gamma r)<\delta<\min \left(\frac{\gamma r\left(1-\Phi^{\prime}\left(W_{\tau_{n}}\right)\right)}{(1-\varepsilon)^{\gamma / \nu}}, r-\gamma r\right)$

If $F_{n}$ as given by (5.18) has the property such that $F_{n} \geq Q_{n}\left(1-R_{n}^{1 / \nu}\right)$, then an optimal policy $(T, W, V, C)$ exists.

Proof : Consider $H\left(T_{n}\right)=g\left(T_{n}\right)+\left(X_{\tau_{n}}-Y_{n}\right) h\left(T_{n}\right)$. By Remark 5.2 $\frac{A_{n+1}}{Q_{n+1}} \geq \frac{R_{n}\left(1-R_{n}^{1 / \nu}\right)}{\left(1-\Phi^{\prime}\left(W_{\tau_{n}}\right)\right)^{1 / \nu}}$, for $F_{n} \geq Q_{n}\left(1-R_{n}^{1 / \nu}\right)$. By Theorem 5.3, then there exists a scalar $\hat{T}_{n}>0$ such that $H\left(\hat{T}_{n}\right)=0$. The arguments in the proof of Theorem 5.3 show that one of the solutions, say $\hat{T}_{n}$, corresponds to the maximum of (5.10).

Now consider the following withdrawal and investment policy and function $f$, for $n=1,2,3, \ldots$,

$$
f\left(X_{\tau_{n}}\right)=Q_{n}^{\nu} A_{n}^{-\nu} \frac{1}{\gamma}\left(X_{\tau_{n}}-Y_{n}\right)^{\gamma},
$$




$$
\begin{aligned}
W_{\tau_{n}} & =F_{n}\left(X_{\tau_{n}}-Y_{n}\right)+\Phi\left(W_{\tau_{n}}\right) \\
V_{\tau_{n}} & =(1-\varepsilon)\left(1-F_{n}\right)\left(X_{\tau_{n}}-Y_{n}\right) \pi_{n} \\
T_{n} & =\hat{T}_{n} .
\end{aligned}
$$

Lemma 5.3 shows that $f\left(X_{\tau_{n}}\right)$ satisfies $(5.10)$ and $\left(T_{n}, W_{\tau_{n}}, V_{\tau_{n}}\right)$ achieves the maximum in (5.12) for all $n=1,2,3, \ldots$

Now it will be shown that $\lim _{n \rightarrow \infty} E\left[e^{-\delta \tau_{n}} f\left(X_{\tau_{n}}\right)\right]=0$. Since $\tau_{n+1}=$ $T_{n}+\tau_{n}$, then by using (5.60) we have that

$$
E\left[e^{-\delta \tau_{n+1}} f\left(X_{\tau_{n+1}}\right) \mid \mathcal{H}_{\tau_{n}}\right]=Q_{n+1}^{\nu} A_{n+1}^{-\nu} \frac{1}{\gamma} e^{-\delta\left(T_{n}+\tau_{n}\right.} E\left[\left(X_{\tau_{n+1}}-Y_{n+1}\right)^{\gamma} \mid \mathcal{H}_{\tau_{n}}\right] .
$$

But according to relation (5.11) upon insertion of $W_{\tau_{n}}$ and $V_{\tau_{n}}$ as given by relations (5.61) and (5.62) respectively, $X_{\tau_{n+1}}$ may be written as

$$
X_{\tau_{n+1}}=(1-\varepsilon)\left(1-F_{n}\right)\left(X_{\tau_{n}}-Y_{n}\right)\left[e^{r T_{n}}+\pi_{n}\left(\Gamma_{n+1}-e^{r T_{n}}\right)\right]+Y_{n+1} .
$$

Therefore, the expectation factor on the right hand side of (5.64) may be written as

$$
E\left[\left(X_{\tau_{n+1}}-Y_{n+1}\right)^{\gamma} \mid \mathcal{H}_{\tau_{n}}\right]=(1-\varepsilon)^{\gamma}\left(1-F_{n}\right)^{\gamma} \Omega_{n}\left[\left(X_{\tau_{n}}-Y_{n}\right)^{\gamma} \mid \mathcal{H}_{\tau_{n}}\right]
$$

This implies that

$$
E\left[e^{-\delta \tau_{n+1}} f\left(X_{\tau_{n+1}}\right) \mid \mathcal{H}_{\tau_{n}}\right]=Q_{n+1}^{\nu} A_{n+1}^{-\nu} \frac{1}{\gamma} R_{n}\left(1-F_{n}\right)^{\gamma} e^{-\delta \tau_{n}}\left[\left(X_{\tau_{n}}-Y_{n}\right)^{\gamma} \mid \mathcal{H}_{\tau_{n}}\right]
$$

Let $D=Q_{n+1}^{\nu} A_{n+1}^{-\nu} \frac{1}{\gamma}$. then by induction we have that

$$
\begin{aligned}
& E\left[e^{-\delta \tau_{n+1}} f\left(X_{\tau_{n+1}}\right) \mid \mathcal{H}_{\tau_{n}}\right] \\
& \quad=D R_{n}\left(1-F_{n}\right)^{\gamma} R_{n-1}\left(1-F_{n-1}\right)^{\gamma} e^{-\delta \tau_{n-1}}\left[\left(X_{\tau_{n-1}}-Y_{n-1}\right)^{\gamma} \mid \mathcal{H}_{\tau_{n-1}}\right]
\end{aligned}
$$




$$
\begin{aligned}
& =D R_{n}\left(1-F_{n}\right)^{\gamma} \ldots R_{1}\left(1-F_{1}\right)^{\gamma} e^{-\delta \tau_{1}}\left[\left(X_{\tau_{1}}-Y_{1}\right)^{\gamma} \mid \mathcal{H}_{\tau_{1}}\right] \\
& \leq D X_{0}^{\gamma} \prod_{k=1}^{n}\left[R_{k}\left(1-F_{k}\right)^{\gamma}\right] \leq D X_{0}^{\gamma} \prod_{k=1}^{n}\left[(1-\varepsilon)^{\gamma / \nu}\right]
\end{aligned}
$$

since $R_{n} \leq(1-\varepsilon)^{\gamma / \nu}$ and $0<1-F_{n} \leq 1$ for any feasible policy. Since $D$ is bounded, then

$$
\lim _{n \rightarrow \infty} E\left[e^{-\delta \tau_{n}} f\left(X_{\tau_{n}}\right)\right]=0
$$

Hence, condition (ii) of the Lemma 5.3 is satisfied. Then by Lemma 5.3 , the proof of Theorem 5.4 has been completed 


\section{Chapter 6}

\section{Conclusions}

We have established a consumption and investment selection problem for an individual who seeks to maximize the expected utility of consumption. The individual has available a riskless asset with fixed interest rate and a risky one with logarithmic Brownian motion price fluctuations. The individual observes current wealth when making transaction, and decisions to transact can be made at any time, but not without costs. The individual is charged a fixed fraction $\varepsilon>0$ of the current wealth as a portfolio management fee plus transaction costs which in general depends on the amount of wealth transacted. Three different types of transaction costs functions were analised, namely, fixed costs, linear costs and nonlinear costs. The problem was formulated in discrete continous time stochastic optimal control problem.

For all types of transaction costs functions, a complete solution to the consumption and investment strategy selection for the individual was derived. The first conjecture in discrete continuous time setting is that it is not optimal 
for an individual to take more money out of his/her portfolio than it is needed for consumption during intervals. This conjecture applies for the three types of transaction costs functions.

For given transactions intervals, we derived the optimal value function as well as the optimal strategy for the withdrawal process and the investment in the risky asset. We showed that, for each interval, the optimal value function as well as the optimal withdrawal process and the investment strategy in the risky asset were obtained by dynamic programming.

Another result is that for all types of transaction costs, the optimal transaction intervals $T_{n}$ depend on the amount of total wealth at the beginning of each intervals.

If, for any reason that transactions do not incur costs other than those of management fee, it was shown that the optimal interval between transactions are independent of the wealth at the beginning of the interval. This result verifies those of Duffie and Sun [7]. This is so because Duffie and Sun [7] actually treated linear transaction costs by transforming it to fixed transaction costs problem. Then by taking $b=0$, Duffie and Sun [7] provide result only for the proportional transaction cost problem. 


\section{Bibliography}

[1] V. M. Alekseev, V. M. Tikhomirov, and S. V. Fomin. Optimal Control. Consultants Bureau, New York, 1987.

[2] George M. Constantinides. Multiperiod consumption and investment behavior with transaction costs. Management Science, 25(11):1127-1137, November 1979.

[3] G.M. Constantinides. Capital market equilibrium with transaction costs. Journal of Political Economy, 94(4):842-862, 1986.

[4] J.C. Cox and C.F. Huang. Optimal consumption and portfolio policies when asset prices follow a diffusion process. J. Econ. Theory, 49:33-83, 1989.

[5] John C. Cox and Chi-fu Huang. A variational problem arising in financial economics. Journal of Mathematical Economics, (20):465-487, 1991. 
[6] M.H.A. Davis and A.R. Norman. Portfolio selection with transaction costs. Mathematics of Operations Research., 15(4):676-713, November 1990.

[7] Darrell Duffie and Tong-sheng Sun. Transactions costs and portfolio choice in a discrete-continuous-time setting. Journal of Economic Dynamics and Control, 14:35-51, 1990.

[8] B. Dumas and E. Luciano. An exact solution to a dynamic portfolio choice problem under transactions costs. Journal of Finance, XLVI(2):577-595, June 1991.

[9] Frank J. Fabozzi. Investment Management. Prentice Hall Inc., New Jersey, 1995.

[10] W. H. Fleming and T. Zariphopoulou. An optimal investment and consumption model with borrowing. Mathematics of Operations Research, 16(4):802-822, November 1991.

[11] Nils H. Hakansson. Optimal investment and consumption strategies under riks for a class of utility functions. Econometrica, 38(5):587-607, September 1970.

[12] I. Karatzas and S.E. Shreve. Brownian Motion and Stochastic Calculus. Springer-Verlag, New York, 1988.

[13] Ioannis Karatzas. Optimization problems in the theory of continuous trading. SIAM J. Control and Optimization, 27(6):1221-1259, November 1989 . 
[14] Ioannis Karatzas et al. Explicit solution of a general consumption and investment problem. Mathematics of Operations Research, 11(2):261294, May 1986.

[15] J. Lehoczky, S. Sethi, and S. Shreve. Optimal consumption and investment policies allowing consumption constraints and bankruptcy. Mathematics of Operations Research, 8(4):613-636, November 1983.

[16] Hayne E. Lelands. Option pricing and replication with transactions costs. The journal of Finance, XL(5):1283-1301, December 1985.

[17] Michael J.P. Magill and George M. Constantinides. Portfolio selection with transactions costs. Journal of Economic Theory, 13:245-263, 1976.

[18] Robert C. Merton. Continuous-Time Finance. Blackwell, Cambridge, 1990.

[19] Robert C. Merton. Optimum consumption and portfolio rules in continuous time model. Journal of Economic Theory, 3:373-413, 1971.

[20] Jan Mossin. Optimal multiperiod portfolio policies. The Journal of Business, 41(2):215-229, April 1968.

[21] S. R. Pliska. A discrete time stochastic decision model. Lecture Notes in Control and Informaton Sciences, 42:290-304, 1982.

[22] Phillip Protter. Stochastic Integration and Differential Equations. Springer-Verlag, Berlin, 1990. 
[23] Paul A. Samuelson. Lifetime portfolio selection by dynamic stochastic programming. Review of Economics and Statistics, 51:239-246, 1969.

[24] S.P. Sethi, M.I. Taksar, and E.L. Presman. Explicit solution of a general consumption and portfolio problem with subsistence consumption and bankruptcy. Journal of Economics Dynamics and Control, 16:747-768, 1992.

[25] S.E. Shreve and H.M. Soner. Optimal Investment and Consumption With Transaction Costs. Research Report, Carnegie Mellon, September 1992.

[26] S.E. Shreve, H.M. Soner, and G.-L. Xu. Optimal investment and consumption with two bonds and transaction costs. Mathematical Finance, 1(3):53-84, July 1991.

[27] Michael Taksar, Michael J. Klass, and David Assaf. A diffusion model for optimal portfolio selection in the presence of brokerage fees. Mathematics of Operations Research, 13(2), May 1988.

[28] Thalea Zariphopoulou. Consumption-investment models with constraints. SIAM J. Control and Optimization, 32(1):59-85, January 1994.

[29] Thalea Zariphopoulou. Investment-consumption models with transaction fees and markov-chains parameters. SIAM J. Control and Optimization, 30(3):613-636, May 1992. 